\title{
Validity and reliability of accelerometers for examining vertical jump performance
}

\author{
Ryan M. Ruben \\ West Virginia University
}

Follow this and additional works at: https://researchrepository.wvu.edu/etd

\section{Recommended Citation}

Ruben, Ryan M., "Validity and reliability of accelerometers for examining vertical jump performance" (2010). Graduate Theses, Dissertations, and Problem Reports. 4650.

https://researchrepository.wvu.edu/etd/4650

This Thesis is protected by copyright and/or related rights. It has been brought to you by the The Research Repository @ WVU with permission from the rights-holder(s). You are free to use this Thesis in any way that is permitted by the copyright and related rights legislation that applies to your use. For other uses you must obtain permission from the rights-holder(s) directly, unless additional rights are indicated by a Creative Commons license in the record and/ or on the work itself. This Thesis has been accepted for inclusion in WVU Graduate Theses, Dissertations, and Problem Reports collection by an authorized administrator of The Research Repository @ WVU. For more information, please contact researchrepository@mail.wvu.edu. 
Validity and reliability of accelerometers for examining vertical jump performance

Ryan M. Ruben

Thesis submitted to the
School of Medicine
at West Virginia University
in partial fulfillment of the requirements
for the degree of

Master of Science

in

Exercise Physiology

G. Gregory Haff, Ph.D., Chair Jean L. McCrory, Ph.D.

Randy W. Bryner, Ed.D.

Department of Human Performance

Division of Exercise Physiology

Morgantown, West Virginia

2010

Copyright 2010 Ryan M. Ruben

Keywords: Performance Testing, Athlete Monitoring, Power, Countermovement Jump 


\title{
ABSTRACT \\ Validity and reliability of accelerometers for examining vertical jump performance
}

\author{
Ryan M. Ruben
}

PURPOSE: The objectives of this study were to determine the validity and reliability of an accelerometer as a device for estimating force-time curve characteristics and examine the effect of reducing sampling frequency.

METHODS: Sixty college aged men and women (age $=23.6 \pm 3.1 \mathrm{y}$; height $=180.1 \pm 6.3 \mathrm{~cm}$; weight $=85.0 \pm 15.2 \mathrm{~kg}$; body fat $=14.2 \pm 6.5 \%$ ) performed 10 restricted (no arm swing) zero-load countermovement vertical jumps each for a total of 600 jumps. Peak force, rate of force development, peak power output, peak velocity, flight time and peak vertical displacement were assessed with the use of a tri-axial accelerometer and compared to a force platform + linear position transducer system. The data from the accelerometer were then resampled to determine the optimum sampling frequency. Reliability was assessed by intraclass correlation (ICC) and coefficient of variance (CV). Validity was evaluated by a linear regression analyses to determine a calibration equation, the standard error of the estimate (SEE) and a validity correlation coefficient.

RESULTS: The accelerometer was found to be reliable for peak force, peak power, peak velocity and peak displacement for each sampling frequency. In regards to validity, the accelerometer significantly overestimated peak force, peak rate of force development, peak power and displacement while it underestimated peak velocity.

CONCLUSIONS: The accelerometer was found to be a reliable device at frequencies as low as $50 \mathrm{~Hz}$, with the best validity at $250 \mathrm{~Hz}$; therefore, $250 \mathrm{~Hz}$ is an acceptable sampling frequency when testing with accelerometers. The accelerometer overestimates some variables, which may be due to including bodyweight in the calculations.

KEY WORDS: Performance Testing, Athlete Monitoring, Sport Science, Power, Countermovement Jump 


\section{Acknowledgements}

First of all, I wish to thank;

Dr. G. Gregory Haff, Dr. Jean L. McCrory and Dr. Randall W. Bryner for their supervision.

I would like to thank all my subjects for participating in this research project.

Additionally, I would like to thank Heather Saffel, West Virginia University and Dr. Prue Cormie Edith Cowan University for their assistance as co-investigators. Furthermore, I must thank Dr. Michael H. Stone, East Tennessee State University and Dr. William A. Sands, U.S. Olympic Committee for their support.

Also, I would like to acknowledge the accelerometers in this project were donated by Myotest, Inc.

Finally, I would like to thank my parents W. Dale, Camillia and Laura Ruben, my grandparents Jon and Sue Shank, and W. Wesley (deceased) and Shirley (deceased) Ruben, my brother and sister Jon Ruben and Molly Robinson, and my girlfriend Alyssia Hall for all of their support. 


\section{$\underline{\text { Dedication }}$}

This thesis is dedicated to all sport scientists who devote their lives to making science an integral part of training athletes. 


\section{Table of Contents}

Section $\underline{\text { page }}$

Chapter 1. Specific Aims and Hypothesis ............................................................................... 1

1.1. Central Hypothesis.......................................................................................................... 3

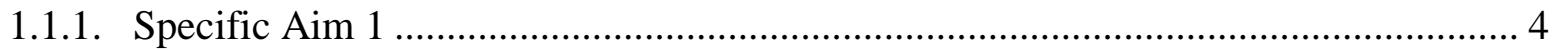

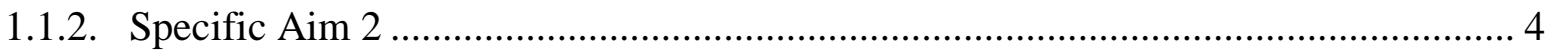

1.2. Operational Definitions ......................................................................................................... 5

Chapter 2. Background \& Significance ........................................................................ 7

2.1. Introduction .............................................................................................................................. 7

2.2. Determinants of Jump Performance ............................................................................ 12

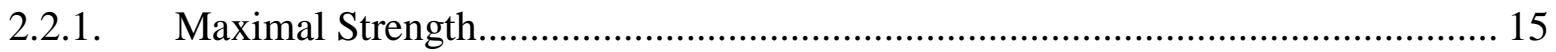

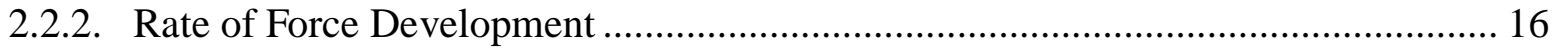

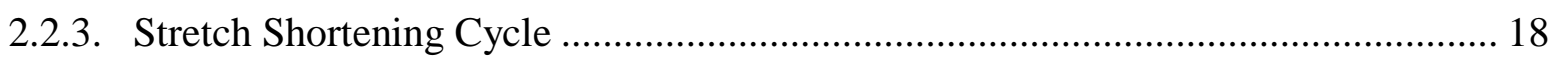

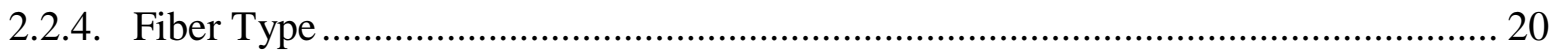

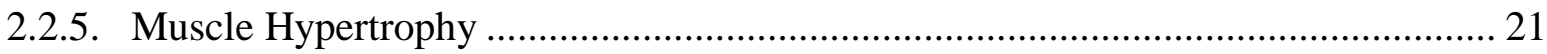

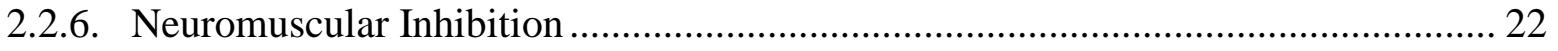

2.2.7. Motor Unit Recruitment.............................................................................. 22

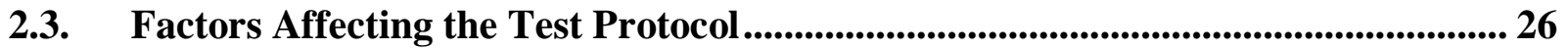

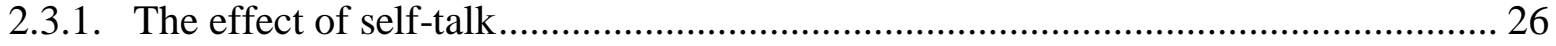

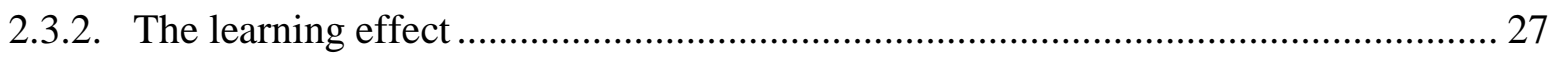

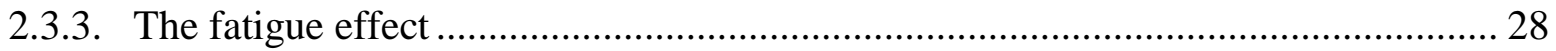

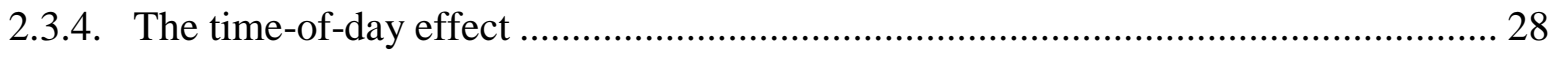

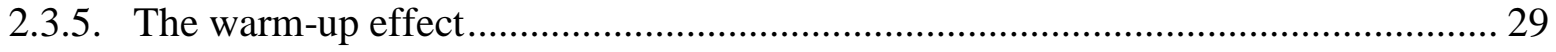

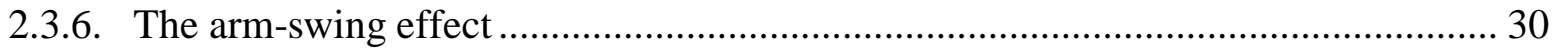


2.4. Methods and Tools Measuring Human Force-time Curve Variables..................... 32

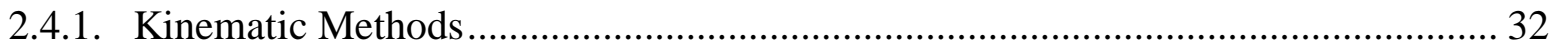

2.4.2. Kinetic Methods........................................................................................ 38

2.4.3. Kinematic + Kinetic Methods .................................................................. 43

2.4.4. Contact Time Methods .................................................................................. 46

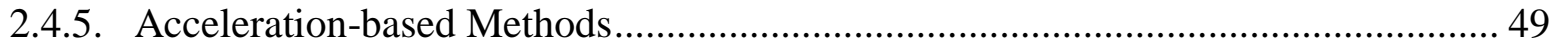

2.4.6. Summary of Methods and Tools Measuring Human Force-time Curve Variables .. 56

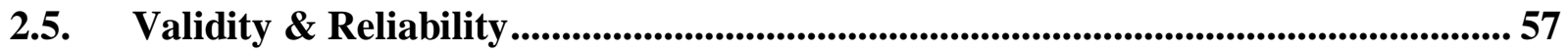

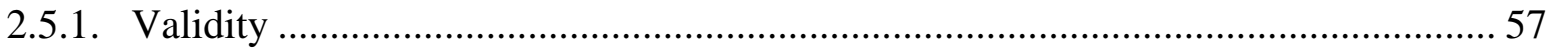

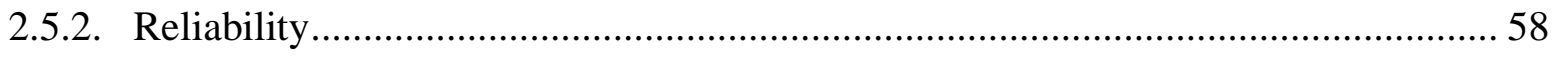

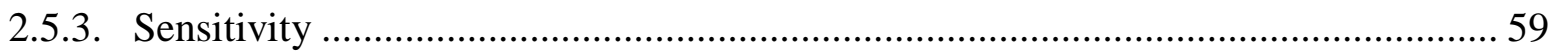

2.6. Summary and Implications of Literature Review ......................................... 60

Chapter 3. Manuscript written in MSSE style of all data ........................................... 83

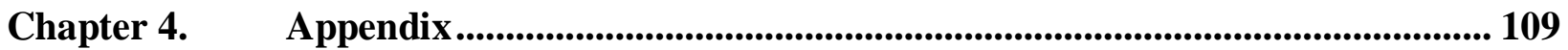


List of Tables

Table 3.1: Reliability Data for the Accelerometer and Force Platform + Linear Position

Transducer System for the Total Sample Population.

Table 3.2: Reliability Data for the Accelerometer and Force Platform + Linear Position

Transducer System for the Men.

Table 3.3: Reliability Data for the Accelerometer and Force Platform + Linear Position

Transducer System for the Women.

Table 3.4: Linear Regression Equations between Force Platform + Linear Position

Transducer and Accelerometer Data.

Table 3.5: Linear Regression Equations between Force Platform + Linear Position

Transducer and Accelerometer Data for Men and Women.

Table 3.6: Vertical Jump Data for Men and Women 


\section{List of Figures}

Figure 2.1: A mathematical outline of the LPT method...................................................................... 35

Figure 2.2: A mathematical outline of the 2-LPT method ............................................................... 36

Figure 2.3: A mathematical outline of the force platform method.............................................. 40

Figure 2.4: A mathematical outline of the force platform + single LPT method.................... 44

Figure 2.5: A mathematical outline of the force platform + 2-LPT method ............................ 45

Figure 2.6: A mathematical outline of an optoelectronical/switch mat-based method ........ 47

Figure 2.7: Force-time Curve Variables Derived from Acceleration Data .............................. 51

Figure 2.8: A mathematical outline of the acceleration-based method..................................... 52

Figure 4.1 Project Timeline and Design ................................................................................................... 109

Figure 4.2: Force Platform + 2-Linear Position Transducer System with Accelerometer 110

Figure 4.3: Experimental Protocol ................................................................................................. 111

Figure 4.4: Correlation analysis between peak forces from accelerometer and force plate

data

Figure 4.5: Correlation analysis between rate of force development from accelerometer and force plate data.

Figure 4.6: Correlation analysis between peak powers from accelerometer and force plate

data

Figure 4.7: Correlation analysis between peak velocities from accelerometer and force plate data.

Figure 4.8: Correlation analysis between flight time from accelerometer and force plate data

Figure 4.9: Correlation analysis between vertical displacements from accelerometer and force plate data. 
Figure 4.10: Correlation analysis between peak forces from accelerometer and force plate data for the women's sub-group 118

Figure 4.11: Correlation analysis between RFD from accelerometer and force plate data for the women's sub-group 119

Figure 4.12: Correlation analysis between peak powers from accelerometer and force plate data for the women's sub-group 120

Figure 4.13: Correlation analysis between peak velocities from accelerometer and force plate data for the women's sub-group

Figure 4.14: Correlation analysis between flight time from accelerometer and force plate data for the women's sub-group

Figure 4.15: Correlation analysis between flight time from accelerometer and force plate data for the women's sub-group

Figure 4.16: Correlation analysis between peak forces from accelerometer and force plate data for the men's sub-group. 124

Figure 4.17: Correlation analysis between RFD from accelerometer and force plate data for the men's sub-group 125 Figure 4.18: Correlation analysis between peak powers from accelerometer and force plate data for the men's sub-group. 126 Figure 4.19: Correlation analysis between peak velocities from accelerometer and force plate data for the men's sub-group 127 Figure 4.20: Correlation analysis between flight time from accelerometer and force plate data for the men's sub-group. 128 
Figure 4.21: Correlation analysis between vertical displacements from accelerometer and

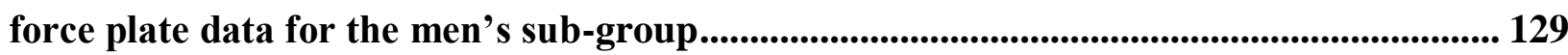




\section{Chapter 1. Specific Aims and Hypothesis}

The vertical jump test is an easily measured activity that has been observed to have mechanical similarities to weightlifting movements $(28,35,63)$ as well as other athletic activities $(16,127)$. For example, the vertical jump has been positively correlated to the squat, snatch, and clean and jerk $(1,28)$, and other markers of performance, such as sprinting (63), agility (35), maximal running velocity (16), and maximal strength (127). One of the benefits of the vertical jump test is it can be carried out quickly and with relatively little interference with training (186). The results of such field tests can provide the coach with valuable information about the potential of the athlete, track fluctuations in fatigue and preparedness that occur during a periodized training program, and help provide information about the athlete that can be used to guide the training program (29).

There are various ways to estimate vertical jump height including single or multiple linear position transducers (29), force platforms $(31,67,123)$, combinations of linear position transducers and a force platform (41), accelerometers (31), jump-and-reach methods (30), switch mats $(30,87,120)$, and optoelectronical systems $(44,49)$. Although these devices have been shown to be reliable, some are not valid and each has limitations that affect their usefulness. For example, the switch mat, optoelectronical system, and the jump and reach method fail to give information about force-time curve characteristics including force, power, and velocity. In order to quantify these values, an accelerometer, force platform, linear position transducer system or some combination of these two methods must be utilized (171). While the force platform and linear position transducer systems give insight into force-time characteristics, they are expensive, hard to transport, and often require a degree of technical skill that most coaches do not posses $(81)$. 
Recently, the use of accelerometers to test vertical jump height has become increasingly popular. However, the information given from the accelerometer must be valid and reliable to effectively aid the coach in improving athletic performance $(29,118)$. One study has been performed on the validity and reliability of accelerometers; however, this study was performed against an optoelectronical and video system. Currently, a force platform + linear position transducer system is considered to be a gold standard for measuring force-time curve variables, such as those seen in the vertical jump test. Therefore, a study is needed to compare the results given by the accelerometer against those given by the force platform + linear position transducer system.

Sampling frequency can be defined as how many samples per second the device can collect. Generally, a high sampling frequency, such as that seen in a force platform or with a linear position transducer, is more expensive because it generates larger data files and thus more disk storage space and processing time (191). Conversely, a device, such as an accelerometer, with high portability usually possesses a lower sampling frequency (81). This lower sampling frequency could allow for more files to be stored on each unit and enable quicker upload times. However, lowering the sampling frequency can also affect the validity and reliability of the device (81). Thus, it is important for the sport scientists and strength \& conditioning coaches to consider what would be the minimum required sampling frequency for measuring force-time curve variables during the vertical jump. Hence, the central purposes of this thesis are to determine the optimal sampling frequency of an accelerometer system and determine if it is a valid and reliable tool for estimating power, force, velocity, and jump height during a countermovement vertical jump test when compared to a force platform and linear position transducer system. 


\subsection{Central Hypothesis}

This study will examine the validity and reliability of accelerometers in estimating

performance measures such as power, force, velocity, and jump height during a

countermovement vertical jump test. In addition, this study will determine the optimum sampling frequency for accelerometer systems. The central hypothesis of this thesis is that accelerometers are reliable and valid, but not precise instruments for estimating force, RFD, velocity, power output, flight time and displacement during a CMJ test when compared to a force platform + LPT system. That hypothesis is based on the following observations. First, accelerometer systems have been shown to accurately detect the instants when the maximal positive vertical velocity during the takeoff and the maximal negative velocity during the landing are reached; however, it does not accurately measure the absolute value of the acceleration and consequently the velocity (81). In addition, although performed on a force platform, Hori (30) showed that eight different force-time curve variables satisfied a minimum acceptable reliability when collecting at $500 \mathrm{~Hz}$. The central hypothesis will be tested by the following specific aims. 


\subsubsection{Specific Aim 1}

The first aim was to determine if an accelerometer system estimates reliable and valid performance measurements when compared to a force platform and 2-linear position transducer system

Hypothesis: It was hypothesized that the accelerometer system is reliable and valid, but not precise in estimating force, RFD, velocity, power, flight time and displacement during a countermovement vertical jump test when compared to a force platform and linear position transducer system.

\subsubsection{Specific Aim 2}

The second aim was to determine the influence of sampling frequency on the reliability and validity of the accelerometer system to estimate force, RFD, velocity, power, flight time and jump displacement values.

Hypothesis: It was hypothesized that reducing the sampling frequency below $250 \mathrm{~Hz}$ would negatively affect the reliability and validity of data collected with the accelerometer system. 


\subsection{Operational Definitions}

Accelerometer - Device used to measure acceleration, from which variables such as force, power, velocity, and jump height can be calculated (81).

Countermovement Jump - Used to measure the reactive strength of the lower body and involves the athlete squatting until their thigh is parallel to the ground and then immediately jumping upward attempting to maximize the height jumped $(136,168)$.

Force - Synonymous with strength; results in an object's acceleration $(\mathrm{F}=\mathrm{ma})$ when exerted on an external object having a mass (190).

Fiber Type - The three different types of skeletal muscle in the body including type I (slowest), type IIA, and type IIB/x (fastest) (168).

Flight Time - Time that the subject is in the air and is quantified as the time when takeoff occurs until the time when landing occurs (58).

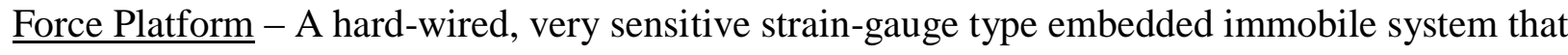
is the accepted standard for force measurement research and commonly used to measure jumping and landing tasks (30).

Linear Position Transducer - A device that measures voltage output, which can be converted to a displacement and put into various equations to determine power, force, and velocity (181). Muscle Hypertrophy - An increase in size of the muscle (31).

Motor Unit Recruitment - Activating the motor nerve and all of the muscle fibers it innervates (23).

Neuromuscular Inhibition - A protective mechanism that provides feedback from various muscle and joint receptors, which can reduce muscle tension during maximum and near-maximum efforts (23). 
$\underline{\text { Power }}-\mathrm{A}$ unit of work expressed per unit of time (i.e., power $=$ work $\cdot$ time $^{-1}$ ), often considered a factor of intensity. May also be calculated by multiplying force x velocity (168).

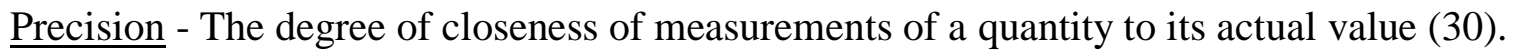

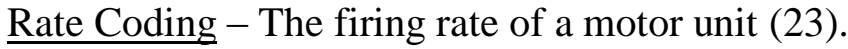

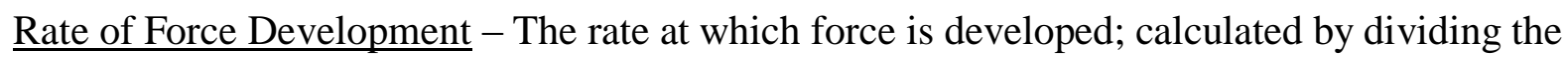
change in force by the change in time (23).

Reliability - The degree of consistency of measurement (23).

Sampling Frequency - How often the signal is sampled each second (168).

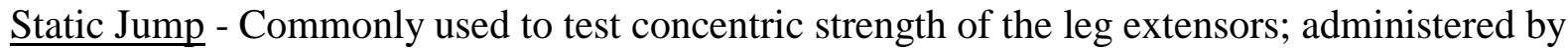
the subject starting in the bottom position (i.e., top of the thigh parallel to the ground) and then jumping upward (125).

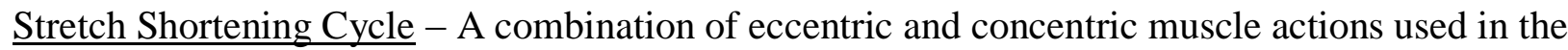
countermovement jump (190).

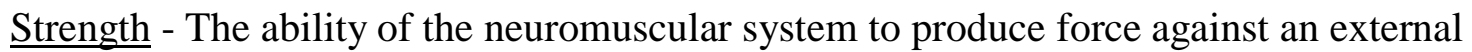
resistance $(23,156)$.

Validity - Whether or not the instrument is actually measuring what it is supposed to be measuring (163).

Velocity - The speed of movement of the body of an object. It is calculated by dividing distance by time (168). 


\section{Chapter 2. Background \& Significance}

\subsection{Introduction}

The ability to produce power is an integral part of many individual and team sports (23) including American football, track \& field, basketball, and soccer (135). Athletes with a higher playing ability generally have been shown to produce higher power outputs in a variety of sports $(12,186)$. Because of this relationship, power output may be the most important characteristic in sport, and for this reason it becomes critical to periodically test (144). In competitive athletes, power must be developed through a long-term strategy based on the theory of periodization (168). Strength and conditioning coaches and sports scientists must carry out performance tests throughout these training programs in order to monitor progress, prevent overtraining, and modify training stimuli. Thus, valid and reliable tests are required to assess the athlete's explosive power generating capacity at various time points throughout the training program. Essentially, these tests are used to determine the athlete's level of preparedness and to determine how they are responding to the training program.

The vertical jump test is one of the more popular field test approaches $(138,179)$ used by coaches, health care professionals, and strength and conditioning coaches to assess lower body power output. The vertical jump test determines displacement, which is then used to estimate power output. Numerous coaches consider the vertical jump to be an essential skill that contributes to higher performance in various sports such as American football, basketball, diving, and volleyball (111). Additionally, vertical jump ability is frequently assessed to measure improvement of an athlete's capabilities throughout a specific training program (87, 111). Furthermore, the ability to express high power outputs is also required when attempting to rapidly change direction or accelerate during various sports or athletic events (111). Barnes et al. 
(135) indicate that individuals with greater countermovement jump performance also have quicker agility times $\left(\mathrm{R}^{2}=0.34\right)$. Thus, the vertical jump test can also examine the ability to change direction.

There are two different ways to perform the vertical jump: the countermovement jump and static jump (16). The countermovement jump is used to measure the reactive strength of the lower body (74) and involves the athlete dipping down until their thigh is parallel to the ground and then immediately jumping upward attempting to maximize the height jumped (190). This type of jumping method utilizes the stretch-shortening cycle, which combines eccentric/concentric muscle actions to elicit a higher jumping ability $(106,136,158)$. Conversely, the static jump is commonly used to test the concentric strength of the leg extensors (104). In the static jump, the subject starts in the bottom position and then jumps upward without engaging the stretch shortening cycle. The use of these exercises to test power production is based upon the fact that a better performance can be produced when high power outputs are produced $(29,190)$. The countermovement jump has shown the greatest reliability among jumping tests $(\mathrm{ICC}=0.98)(29,32)$.

In the past, the common method to calculate power output from a vertical jump test was to use the Lewis formula, which gives the average power exerted by gravity on the jumper's body during the falling phase (120). The Lewis formula was derived from standard equations of projectile motion (74). These equations describe the fall of the jumper's center of mass from its high point to where the foot contacts the ground (74).

$$
\mathrm{H}=\mathrm{V}_{\mathrm{o}} \cdot \mathrm{t} \pm 0.5 \cdot \mathrm{g} \cdot \mathrm{t}^{2}
$$

Vertical center of mass velocity is zero at the highest point of the jump, thus:

$$
\mathrm{H}=0 \cdot \mathrm{t}+1 / 2 \cdot 9.8 \cdot \mathrm{t}^{2}
$$


This equation can be reduced to:

$$
\mathrm{t}=\overline{-}
$$

Since average velocity of the center of mass is distance divided by time, the following equation can be formed:

$$
\mathrm{V}_{\mathrm{AV}}=-
$$

Then, $\stackrel{-}{=}$ can be substituted for $\mathrm{t}$ :

$$
\mathrm{v}_{\mathrm{AV}}=\quad \overline{-}=-\overline{-}
$$

Furthermore, power is equal to force times velocity:

$$
\mathrm{P}_{\mathrm{AV}}=\text { force } \cdot \mathrm{V}_{\mathrm{AV}}
$$

Body mass times the acceleration due to gravity can be substituted for force and $\sqrt{\mathrm{H}} \cdot \sqrt{4} .9$ can be substituted for average velocity. This gives an exact equation equal to the Lewis formula:

$$
\mathrm{P}_{\mathrm{AV}}=9.8 \cdot \text { body mass }(\mathrm{kg}) \cdot-\cdot-
$$

The Lewis formula gives the power exerted by gravity on the jumper's body during the falling phase (74)

$$
\left.{ }^{-1}\right)=-
$$

The Lewis formula was updated by Harman et al. (74) due to several key problems. First, it only gave the power output of the falling phase and not the power generated during takeoff. In addition, the formula did not use standard units. Furthermore, the equation did not specify whether it was peak power or average power. To address these issues, Harman et al. (74) created the following equations by way of multiple regressions (74):

$$
\begin{gathered}
\text { Peak power }(\mathrm{W})= \\
\text { Average power }(\mathrm{W})=
\end{gathered}
$$


Because of the small sample size used by Harmon $(n=17)$, the validity of the equation was examined by Sayers et al. (74). They cross-validated both the Lewis formula and Harmon equation with 108 college-age male $(n=59)(21.3 \pm 3.4$ years $)$ and female $(n=49)(20.4 \pm 2.2$ years $)$ subjects against a force platform. Cross-validation was performed by entering the variables of jump height and body weight of the 108 subjects into the two equations of Lewis (152) and Harman et al. (74) and comparing the estimates of peak and average power from the respective equations to actual peak and average power determined from the force platform. Peak power was underestimated by $6.9 \%(r=0.87)$ when using countermovement jump data in the equation of Harman et al. (74) and by $72.8 \%(r=0.87)$ in the Lewis formula (74). The Sayers Equation was then developed to estimate peak power output (Peak Anaerobic Power output or PAPw) from the vertical jump and is frequently used by coaches and in the scientific literature (74): $\mathrm{PAPw}(\mathrm{W})=60.7 \cdot$ body displacement $(\mathrm{cm})+45.3 \cdot$ body mass $(\mathrm{kg})-2,055$

Johnson and Bahamonde (152) also developed a formula for the calculation of peak and average power from the vertical jump test, using the countermovement jump. They tested 118 college age (avg. age $=19.58$ years \pm 1.24$)$ males $(n=69)$ and females $(n=49)$ on a combination of a force platform and a Vertec. The Vertec is a vertical jump testing device made of a steel frame with horizontal vanes along the top that are rotated out of the way by the hand of the athlete to determine displacement. Johnson and Bahamonde's equations use the additional factor of body height. Although height was a significant variable entered in both equations, it did not produce significant changes in the accounted percentage of variance. Also, there were no significant changes to the standard error of estimate of both equations. Both equations had strong correlations with actual peak and average power taken from the force platform $\left(\mathrm{R}^{2}=0.916\right.$ and 0.831$)$ 
Peak Power $(\mathrm{W})=78.6 \cdot$ body displacement $(\mathrm{cm})+60.3 \cdot$ body mass $(\mathrm{kg})-15.3 \cdot$ height $(\mathrm{cm})-1,308$ Average Power $(\mathrm{W})=43.8 \cdot$ body displacement $(\mathrm{cm})+32.7 \cdot$ mass $(\mathrm{kg})-16.8 \cdot$ height $(\mathrm{cm})+431$

However, even with prediction equations, standard vertical jump tests do not provide feedback about important variables such as peak force, acceleration, velocity, rate of force development, and rate of power development. These parameters can be identified with the use of more technical measurements performed on a force platform and/or with linear position transducers $(31,96)$. The force platform provides information about the magnitude and direction of forces, torques applied to the surface of the force platform, and center of pressure (83). While these instruments are highly reliable and produce valid results, they are very expensive and difficult to transport to the strength \& conditioning facility or field locations, which limits their applicability (118); thus, warranting devices that can accommodate portability and are reasonably priced.

A relatively newer way of estimating force-time curve characteristics is the use of accelerometers (118). The accelerometer measures vertical acceleration and then derives other variables such as power, force, and velocity from that measure. These variables can be provided immediately after the jump is performed; thus, allowing the device to operate as a field test. Accelerometers can allow for immediate feedback of force-time characteristics in the training setting without the need to go to a laboratory or to use expensive measurement instruments with complex software that are difficult to transport. Therefore, they could be considered an "advanced field test." However, for this data to be useful for sport scientists and strength \& conditioning coaches, the data must be valid and reliable. For example, if the feedback is not reliable, the person responsible for the program design may make unnecessary changes to the training program thinking the athlete is more or less fatigued than the athlete actually is. Validity is equally important. For instance, if the sport scientist/strength \& conditioning coach is 
using the accelerometer to measure power output, they must have confidence that they are actually receiving valid power outputs from the accelerometer.

Accelerometers generally sample at either $250 \mathrm{~Hz}$ or $500 \mathrm{~Hz}$ (30), which is considerably less than the $1000 \mathrm{~Hz}$ sampling rate typically being used with a force platform or linear position transducers (30). Although some of the feedback generated by the accelerometer appears to be reliable (31), recent work by Hori et al. (30) suggests that lower sampling rates performed on a force platform results in an overestimation of power output in the vertical jump. Thus, it is possible that the lower sampling rate typically used by accelerometers may contribute to measurement errors. There is a lack of available research literature on the sampling frequency used during performance measurements with accelerometers. Therefore, determining the effect of reducing sampling frequency and, perhaps more importantly, the minimum sampling frequency that can be used for this form of performance analysis is an important consideration for both sport scientists and strength \& conditioning coaches (82).

The primary aim of this thesis is to determine the reliability, validity, and accuracy of accelerometers by comparing them to a combined force platform and linear position transducer system in the assessment of countermovement jump performance. This literature review will demonstrate why force-time curve variables are an integral part of the strength and conditioning program design, the underlying neural and intramuscular mechanisms that contribute to power, force and velocity production, and the ways force-time curve variables are currently assessed in the literature.

\subsection{Determinants of Jump Performance}

Vertical jump height measurement is a simple method that favorably compares with isokinetic testing as a measurement of knee extension power $(81,111)$. The most important parameter to develop a good jump performance is the achievement of the maximal vertical 
velocity at the takeoff position (114). The greater the vertical velocity the subject's center of gravity (COG) achieves at the moment of leaving the ground, the higher the jump the subject will attain (45). Both the force developed by the musculoskeletal system during the propulsive phase of the jump and the control of the movement seems to play an important role in the achievement of maximal jump performance (30).

Support for the importance of the relationship between force generating capacity and vertical jump performance can be seen in the work of Kawamori et al. (22), Carlock et al. (98), and Wisløff et al. (29). Kawamori et al. (186) found strong relationships between isometric peak force and countermovement jump ( $\mathrm{r}=0.82)$. Additionally, Carlock et al. (98) found a strong relationship between 1-RM squat performance and countermovement jump peak power $(\mathrm{r}=0.91$ (men) and $\mathrm{r}=0.82$ (women)). Furthermore, Wisløff et al. (29) found a correlation between maximal half-squat strength and vertical jump performance in elite soccer players. Thus, it is generally accepted that leg strength plays a large role in determining jump performance. Furthermore, heavy weight training can produce an increase in force causing a rightward shift of the force-velocity curve $(69,168,186)$, which occurs in response to an increase in strength. However, high velocity training is also needed to make additional adaptations in the highvelocity end of the force-velocity curve (165). These increases in force and velocity can produce an increase in power output, which is often measured by the vertical jump.

There are two ways to calculate power output. First, power can be expressed as the rate of doing work and is calculated by dividing work by time. Work is the calculated by multiplying force and the distance the object moves in the direction resulting from the force application (168). The force produced in this equation is produced by the previously mentioned leg strength. The other way to calculate power output is by multiplying force and velocity, which can be 
derived from the first equation. Because power is the rate of doing work, it must be performed rapidly to achieve a high power output (1). When performing a countermovement jump, the goal is to achieve the highest peak power that the athlete is capable of attaining (168). Stone (168) defines peak power (PP) as the highest instantaneous power value found over a range of motion. Power can be represented by three possible equations:

$$
\text { Power }=
$$

Force multiplied by distance can be substituted for work:

$$
\text { Power }=
$$

Velocity can be substituted for Distance/Time, thus:

The consensus in the literature is that success in sport performance depends on the ability to produce a high rate of force development and power output, which both contribute to vertical jump performance $(33,109,166-168)$ and contribute to the ability to rapidly change direction or accelerate during various sports or athletic events (11). Therefore, testing these performance characteristics throughout the training plan is paramount. Although, time is often a limiting factor in performance testing, as athletes are often restricted in the amount of time they can spend in the weight room. As stated earlier, accelerometers are a tool used in advanced field testing as they can be used in the weight room or practice facility and can be transported back to the computer with relative ease. Therefore, if proven valid and reliable, accelerometers may prove to be the ideal system for testing athletes' power generating capacity in the practitioner's setting.

There are several non-invasive physiological parameters that are testable and contribute to producing a high power output. These include maximal strength, the rate of force development, and usage of the stretch shortening cycle. Additionally, there are multiple invasive 
physiological parameters that contribute to power output including fiber type, muscular hypertrophy, neuromuscular inhibition, and motor unit recruitment. These invasive variables are not outwardly testable, but have been correlated to power output.

\subsubsection{Maximal Strength}

According to Siff et al. (126) and Stone (135), strength can be defined as the ability of the neuromuscular system to produce force against an external resistance (156). Schmidtbleicher (163) and Stone (168) suggest that maximum strength is the basic quality affecting power output. This is because power is the product of force and velocity, thus alterations in force should alter power production (153). Strength is also an attribute often associated with enhanced sport performance $(166,167)$ and increases in maximal strength have been shown to result in increase vertical jump height and power output in response to strength training $(26,40)$ as well as hypertrophy of type II fibers, increases in the type II/I cross-sectional ratio, and recruitment of higher threshold motor units (167).

Maximal strength contributes to jumping ability in a variety of ways. First, if a given load represents a smaller percentage of maximum, the load will be easier to accelerate if maximal strength levels are higher (164). Secondly, a higher maximum strength level would theoretically correlate to a higher percentage or greater cross-sectional area of type II fibers, which have been shown to strongly contribute to high power outputs; therefore, a higher jumping height will be able to be reached.

Maximal strength can be estimated in the vertical jump by measuring the force output. Kawamori et al. (69) showed a very strong relationship between isometric peak force and countermovement jump height $(r=0.82)$. Since force output is an important component of the countermovement jump, the ability to track changes in force output would be useful to sports scientists and strength \& conditioning coaches. 


\subsubsection{Rate of Force Development}

The rate of force development is defined as the ability to develop force in a short period of time (167). It is calculated by a change in force divided by change in time and is directly related to the rate of increase in muscle activation by the nervous system $(98,135)$. Force is directly responsible for the acceleration of an object; however, Stone (101) has argued that the faster a given force is attained, the corresponding acceleration occurs more rapidly. Thus, rate of force development can be associated with the ability to accelerate objects such as the human body (150). Because of this, the rate of force development appears to be important for sports requiring explosive movements (e.g., sprinting, jumping, and throwing), especially those which require force to be generated during a limited time frame $(\sim 50-250 \mathrm{~ms})(167)$.

Explosive strength can be defined as the rate of force development and is associated with acceleration capabilities (153). McBride et al. (23) suggests that maximal power is synonymous with explosive strength (153). Schmidtbleicher (123) characterized explosive exercise as having maximum or near maximum rate of force development (123). Stone (153) argues that for many sports the ability to produce force rapidly may be more important than actual maximum force production.

The rate of force development parameter has important functional significance in fast and forceful muscle contractions (167). For example, explosive movements such as jumping, change of direction, or punching typically involve contraction times of 50-250 ms (167). Conversely, it typically takes a longer time to reach maximum force in most human muscles (i.e., $300 \mathrm{~ms}$ for the elbow flexors (3) and knee extensors (3)) (169). Thus, the short contraction time may not allow maximal muscle force to be reached during explosive muscle actions and, as a result, any increase in contractile rate of force development becomes highly important as it allows the athlete to reach a higher level of muscle force in the early phase of muscle contraction (e.g., 
within the initial 100-200 ms of contraction) (174). Nevertheless, maximal strength and the rate of force development are interrelated (3) and are both associated with sporting performance (6). The relationship between maximal strength and rate of force development is somewhat of an unknown, but is thought to occur due to alterations in the H-reflex (129). Both of these variables appear to relate to the ability to cause acceleration, which affects movement velocity $(23,78)$.

Viitasalo et al. (168) suggest that countermovement jump is related to the isometric rate of force development. In addition, Kraska et al. (23) found correlations between isometric rate of force development and jump height in both countermovement jump and static jump. Conversely, Haff et al. (177) stated that isometric rate of force development is not related to countermovement jump, however it is strongly correlated with static jump. Similarly, Young and Bilby (109) and Wilson et al. (67) also showed the same lack of relationship. However, a stronger relationship may exist between isometric rate of force development and countermovement jump due to the nature of the training background that athletes incur, which includes a large involvement of the stretch shortening cycle (193). It has been shown that training with explosive exercises such as weightlifting movements can enhance the rate of force development (184). Therefore, the ability to track these changes is an integral part of the program design. The only current way to track rate of force development is through the use of a force platform, linear position transducer, or some combination of the two. However, since force-time curves can be derived from accelerometer data, accelerometers may have the capability to perform this task and could do so with the correct analysis software. Thus, if accelerometers are proven valid and reliable against a force platform + linear position transducer system, tracking rate of force development changes in the field setting could be implemented. 


\subsubsection{Stretch Shortening Cycle}

A stretch shortening cycle can be defined as a combination of eccentric and concentric muscle actions $(70,109)$. The stretch component of the stretch shortening cycle refers to the eccentric muscle action and the shortening component refers to the concentric muscle action $(104,105)$. The eccentric muscle action causes a storage of elastic energy in the tendomuscular system and occurs prior to the concentric muscle action (77), thus it can be termed a plyometric muscle action $(65,142,168)$.

The most recognized purpose of the stretch shortening cycle is the enhancement of the concentric muscle action, which occurs as a result of three things: storage of elastic energy in the series elastic elements, activation of the stretch reflex, and optimization of muscle activation (23). Generally, stretch shortening cycle movements have been classified as fast (100-250ms) and slow (>250ms) (104). Countermovement jumps incorporate a deliberate stretch shortening cycle and enhance vertical jump height and force production compared to static jumps (104, 106, $142,153)$. Since the countermovement jump utilizes a large knee angle $\left(\sim 90^{\circ}\right)$ and therefore uses the entire stretch shortening cycle, it is considered a relatively slower stretch shortening cycle movement (>500ms). It has been suggested that the countermovement jump is a measure of slow stretch shortening cycle ability due to the long duration of the lengthening-shortening contraction $(8,24)$.

There are several ways to estimate the stretch shortening cycle using vertical jump protocols. The stretch shortening cycle usually involves using a pre-stretch movement, such as comparing countermovement jump with static jump performance $(126,190)$. The first way is to measure pre-stretch augmentation (106). This is calculated as a percentage by taking [(countermovement jump-static jump) X static jump $\left.{ }^{-1}\right]$ X 100. 
Another evaluation is to measure reactive strength, which is defined as the ability to utilize stretching of the muscle and change quickly from an eccentric to a concentric contraction (126). Reactive strength expresses athletes' explosive capabilities in dynamic jumping activity (182) and is calculated by subtracting the static jump height from the countermovement jump height (reactive strength $=$ countermovement jump-static jump) (190). Since there is a fast and slow component of the stretch shortening cycle, reactive strength can be divided into slow reactive strength (slow stretch shortening cycle/low stretch loads) and fast reactive strength (fast stretch shortening cycle/high stretch loads) (52). The slow reactive strength is considered to be a measure of the ability to utilize the muscle pre-stretching during the countermovement jump. Fast reactive strength is measured from a drop jump to impose various stretch loads on the leg extensors while using a contact mat, force platform, or accelerometer to measure jump height and contact time. This is considered fast because there is less knee bend and utilizes a faster movement (125-200ms) (190).

The final method often used to estimate the utilization of the stretch shortening cycle is the calculation of the eccentric utilization ratio (190). The eccentric utilization ratio has been suggested as a useful indicator of power performance in athletes (190) and is calculated by dividing the countermovement jump by the static jump (eccentric utilization ratio=countermovement jump/static jump). It has been proposed that the eccentric utilization ratio is an indicator of stretch shortening cycle performance in various sports and during different phases of training (126).

Periodically testing this ratio is important because the ability to utilize the stretch shortening cycle efficiently is a critical factor in many sports. McBride et al. (126) showed that incorporation of stretch shortening cycle exercises into a training program improves power 
production and jump performance. Furthermore, McGuigan et al. (126) suggest that this ratio is sensitive to changes in the type of training being undertaken by the athlete; thus, strengthening the need for valid and reliable devices that can be used in this monitoring process.

\subsubsection{Fiber Type}

The histochemical staining properties of the myosin adenosine triphosphatase (ATPase) enzyme found in the globular region of the myosin head, also known as the myosin S-1 unit, can identify human skeletal muscle fiber types $(124,126)$. In the current literature, three major fiber types are regularly identified: types I, IIA, and IIB/x with type I being the slowest and type IIB/x being the fastest (58). Type II fibers appear to have a greater relationship to strength and power generating capacity (159). Strength and power athletes have been shown to have higher percentages of Type II muscle fibers $(53-60 \%)(23,58,59)$. Individuals with higher type IIa or IIB/x fiber content appear to generate higher forces during high velocity movements as well as higher rate of force developments and this may be partially explained by a higher cross bridge cycling rate (59).

Alterations in the human fiber type profile can be seen with strength training $(1,60)$. There is strong evidence to show that shifts occur from type IIB/x to type IIA in response to strength training $(25,27,54,58,66,75,160)$. Staron et al. (145) studied the time course of adaptations and showed that untrained females can display this conversion with as little as four training sessions.

According to Fry et al. (161), type II fiber concentration is significantly related to vertical jump ability $(\mathrm{r}=0.79)$. Thus, athletes with a higher type II fiber distribution should be able to jump higher than athletes with a lower concentration. Conversely, endurance athletes generally display a greater concentration of type I fibers $(160,162)$, which correspond to higher maximal oxygen consumption rates (59) while having lower force generating capacities. Therefore, 
endurance athletes with a higher type I fiber distribution should have a lower jumping ability than the aforementioned strength power athletes.

\subsubsection{Muscle Hypertrophy}

Almost all strength-training programs produce some form of muscular hypertrophy.

However, it is widely accepted that the increase in force generating capacity early in the resistance-training program is not fully due to an increase in skeletal muscle size $(20,48,84,85$, $103,121,147,170)$. The main increases in the early stages of the resistance-training program are thought to occur due to neural adaptations $(29,32,48,53,68,76,114,152,158)$. After a period of time, an increase in muscle size is seen (133). The increase in the cross-sectional area of trained muscles comes primarily from the increase in size of individual muscle fibers (139). This increase in cross-sectional area of a muscle increases the amount of contractile units and thus increases force-generating capacity $(2,23,117,149)$. This increase in force-generating capacity should result in a higher jumping performance. As stated earlier, there is a strong relationship between force generating capacity and countermovement jump (5). Therefore, stages of the periodized training program may require phases that focus on muscle hypertrophy to increase force generating capacity. As with all phases of the periodized training program, muscle hypertrophy is also dependent on the type, intensity, and volume of strength training (56).

Although all fibers experience some degree of muscular growth (183), type II fibers have the greatest potential for growth due exhibiting a greater plasticity $(5,98)$. When performed simultaneously, endurance training can reduce the hypertrophic response that would normally occur with resistance training alone $(23,58)$, and thus the gains in vertical jump performance typically seen in response to resistance training may be impaired. 


\subsubsection{Neuromuscular Inhibition}

Neuromuscular inhibition can cause a reduction in force production as a result of neural feedback from various muscles and joint receptors $(1,56)$. The Golgi tendon organ causes this effect as it operates as a protective mechanism that prevents the generation of harmful muscular forces during maximal or near-maximal efforts (58). If neural activation patterns of these protective mechanisms are altered, neuromuscular inhibition may be reversed and the protective mechanism may dissipate; thus, allowing the muscle to tolerate a higher threshold and elicit an increase in force-generating capacity that would normally be prevented by organ structures such as the Golgi tendon organ or muscle spindle $(107,168)$.

Aagaard et al. (3) supported this claim by showing that after 12 weeks of resistance training the inhibition of neuromuscular activation was significantly reduced, thus facilitating an increase in force output. Specifically, Gabriel et al. (61) suggest the increase in force generating capacity caused by neuromuscular inhibition in training is due to a down regulation of the $\mathrm{Ib}$ afferent feedback to the spinal motorneuron pool. In addition, the rate of force development has been used as an indirect measure of the neuromuscular function of athletes $(4,23,97)$. Thus, tracking changes in force generating capacity and rate of force development may be useful in detecting changes in neuromuscular function such as neuromuscular inhibition.

\subsubsection{Motor Unit Recruitment}

Motor unit recruitment relates to the number of motor units activated $(61,118,153)$.

When more motor units are activated, the amount of force generated by the muscle increases (23, 157). The priority of selecting motor units is explained by Henneman's Size Principle, which suggests that the size of the motor unit dictates its activation pattern (42). Specifically, larger motor units are activated in response to higher external loads for short durations and smaller motor units are activated in response to lower loads and longer durations $(23,76)$. In most 
instances the upper limit of motor unit recruitment is $\sim 85 \%$ of MVC; however, the upper limit of motor unit recruitment may be as low as $60 \%$ of MVC for some muscles. Desmedt and Godaux (68) found that the recruitment threshold is lowered as the amount of torque is increased. Thus, the motor units with the highest thresholds experience the greatest decrease in recruitment threshold in response to increased torque.

The force generating capacity of the muscle is not constant; it varies in response to speed of contraction, the activation of higher-level motor units, the type of muscle contraction, and the metabolic state of the muscle. For example, motor units are activated earlier during rapid contractions and rapid contractions activate as many as three times as many motor units, which contributes to an increased rate of force development. The type of contraction is also important. For instance, dynamic muscle actions can result in a lowering of the recruitment threshold when compared to isometric muscle actions (76).

Additionally, the metabolic state of the muscle can also change the recruitment pattern (68). Increases in lactic acid result in alterations to group III and IV muscle afferents that may modify motor unit recruitment patterns and change firing rate. Moritani et al. (53) performed a study in which subjects performed repeated contractions at $20 \%$ of MVC for $2 \mathrm{~s}$ followed by $2-\mathrm{s}$ rest for 4 min with either unhindered blood circulation or arterial occlusion given between the 1st and 2nd minutes the metabolic state of the muscle alters motor unit recruitment patterns. They simultaneously recorded intramuscular motor unit spikes and surface electromyogram (EMG) data indicated that mean motor unit spike amplitude, firing frequency and the parameters of surface EMG power spectra remained constant during the experiment with unhindered circulation, providing no electrophysiological signs of muscle fatigue. However, significant increases in mean motor unit spike amplitude and frequency were evident during the contractions 
with arterial occlusion; thus, validating that the metabolic state of the muscle alters motor unit recruitment patterns.

\subsubsection{Motor Unit Synchronization}

Synchronization of motor units involves the simultaneous activation of numerous motor units (43) and has historically been linked to increased force output (46). Semmler (146) demonstrates that increased synchronization of motor units corresponds to higher levels of force output during rapid muscle contractions. However, Yao (132) and Semmler (102) suggest that that motor unit synchronization may not directly enhance force output or maximal strength.

Contemporary theory suggests that synchronization is more related to the rate of force development than the overall force output as motor unit synchronization has been shown to contribute to rate of force development during rapid muscle contractions (128). Therefore, if athletes are able to achieve a higher rate of force development due to motor unit synchronization, they should be able to attain a higher vertical jump since rate of force development and vertical jump have been shown to be highly correlated $(154,155,189)$; thus, reinforcing the idea of monitoring the rate of force development. As mentioned earlier, the theoretical model for monitoring the rate of force development with accelerometers may be ideal for practitioners wishing to perform all testing in the field setting.

\subsubsection{Rate Coding}

Once the upper limit of motor unit recruitment is achieved rate coding is used to increase muscle force. Rate coding deals with the motor unit firing frequency and usually matches the fiber type with Type II fibers depicting a higher firing rate than Type I fibers (154). This attribute of skeletal muscle allows additional force to be generated without recruiting additional motor units (67). It is a strategy for varying force by altering activation frequency by the central nervous system (CNS) and motor neurons and occurs by utilizing temporal summation to induce 
additional force (109). Muscle force varies as a function of activation frequency; thus, the CNS can use rate coding as a method to alter muscle force (177). For example, if high forces are required to move an object or the object itself, the CNS can deliver high-frequency pulses (42). Conversely, if only low forces are required, the CNS can deliver low-frequency pulses (68). Since the rate of motor unit discharge is likely related to the ability to generate force rapidly (113), it may be also related to the rate of force development. Therefore, high-frequency pulses can be delivered to allow for a higher vertical jump by increasing the force output and the rate of force development. 


\subsection{Factors Affecting the Test Protocol}

\subsubsection{The effect of self-talk}

Self-talk can be defined as a way for individuals to increase motivation or enhance a skill by statements made to themselves and has been shown as a way to enhance sport and exercise performance $(46,113)$. There are two types of self-talk in the world of sports performance: motivational self-talk and instructional self-talk $(72,176)$. Motivational self-talk is designed to assist performance by increasing confidence or enhancing skill execution $(175,195)$ while instructional self-talk is designed to facilitate performance by activating a desired movement through correct focus, technique, and strategy execution $(172,175)$.

Tod et al. (175) examined the effects of self-talk on the vertical jump performance. They divided self-talk into four different conditions: motivational self-talk, instructional self-talk, neutral self-talk, and no additional instructions. The following phrases were used for the motivational and instructional conditions: "I can jump high" for motivational and "bend and dive" for instructional. The subject repeated these phrases during the 15 seconds prior to the jump test. The neutral group counted backwards from 1000 and the "no additional information group" wasn't given any information. The results of this study found that instructional and motivational self-talk were associated with significantly higher center-of-mass displacement, greater impulse, and quicker angular rotation about the knee compared with the neutral condition. However, there were no significant differences between instructional and motivational self-talk. Nonetheless, motivational self-talk is highly subjective and difficult to remain constant from subject to subject. Furthermore, instructional self-talk is more likely to produce a consistent result over time. Thus, for a validity and reliability study, instructional selftalk would seem to be the ideal method for consistency, but to also produce a maximal effort that is utilized in the field. 


\subsubsection{The learning effect}

Familiarization trials are often performed before the actual trial to allow the athlete to get used to the motor skills needed to perform the vertical jump (172). Moir et al. (175) found high reliability without the familiarization session depending on the subject's previous training experience. They argue that well-trained athletes do not need a familiarization session for vertical jumps due to the similarity between vertical jumps and a variety of sporting movements. However, they also speculate that untrained sedentary subjects may not have the appropriate motor skills to perform such tasks without appropriate familiarization trials.

This hypothesis is backed by results found by Ploutz-Synder and Giamis (172) as well as Barfield et al. (175). Ploutz-Synder and Giamis (30) found that familiarization sessions were required in untrained young and old women to provide a stable baseline measure of maximum knee extension strength in both young and old women. Additionally, Barfield et al. (130) found it necessary to require at least one familiarization trial in physically active men who did not have a training history in cycling to optimize performance in the Wingate anaerobic test. Thus, a familiarization session should be included in studies that include untrained, sedentary subjects and those that include movements that the subjects are not familiar with in order to account for possible learning effects. 


\subsubsection{The fatigue effect}

A rest interval that allows for full muscular recovery from a single vertical jumping trial to the next is a determinant for the validity of a test. All the jumps have to be performed at maximal effort, avoiding the effect of fatigue (140). Read et al. (15) found that a rest interval length of 15 seconds is sufficient to allow the full muscular recovery after a single maximal vertical jump. They then came to the conclusion that a single vertical jump lasts less than one second, the depletion of the adenosine triphosphate storage is not complete, and its full regeneration takes very little time (140). However, repeated trials of very intense short duration exercises (i.e., 5-10 seconds), such as repeated countermovement jumps, elicit a rapid decrease in the ability to generate power and force, possibly as a result of a reduced capacity to regenerate ATP in response to a depletion of phosphocreatine (PCr) and an accumulation of lactic acid (15, 30, 142). The depletion of PCr stores has been reported to occur during intense power exercises that require as few as 5 to 7 seconds per attempt (142). Rest intervals of 3 to 4 minutes are generally recommended for recovery of the phosphagen energy system (90\%) during highintensity exercises $(71,148)$. Thus, for multiple countermovement jumps, a 3-minute rest interval between sets should allow for recovery of both $\mathrm{PCr}$ and the phosphagen system and has been shown to be effective in the literature.

\subsubsection{The time-of-day effect}

Time-dependent variances, known as circadian rhythms, are variations throughout the solar day of physiological and psychological variables and can have effects on sports performance (178). Variations in jump performance are caused in response to the time of day when the vertical jump test is executed and could cause systematic errors that influence the results if the test is repeated and then compared (178). Bernard et al. (144) confirmed the presence of a timeof-day effect in a continuous anaerobic test such as multiple vertical jumps. They studied 
college age $(23 \pm 3$ years $)$ males $(n=25)$ and recorded flight time and ground contact time from five consecutive jumps on a jump-ergometer to calculate maximal anaerobic power for jumping. The test schedules were at 0900, 1400 and 1800 hours on separate days in random order. The maximal anaerobic power was higher at 1400 and 1800 hours than at 0900 hours. The differences between the morning and the afternoon reached $5 \%-7 \%$ the jump tests $(\mathrm{P}<0.01)$.

Lundeen et al. (108) studied 46 college age males $(n=25)$ and females $(n=21)$ in markers of maximal quadriceps strength, speed of contraction, and muscle power using an isotonic ergometer. They found significant differences in these variables in the afternoon measures compared with the morning measures. In addition, the peak maximal quadriceps strength, speed of contraction, and muscle power in women occurred $\sim 4$ hours earlier in the day compared with the men. Furthermore, they found similar differences in endocrine function measuring cortisol, $\beta$-endorphin, and catecholamines. Therefore, for a validity and reliability test, it is critical to test the subjects at the same time for all sessions to eliminate a time-of-day effect.

\subsubsection{The warm-up effect}

The purpose of a warm-up is to prepare an athlete for training or competition and can improve subsequent performance and lessen the risk of injury (116). The physiological benefits include increased muscle temperature and core temperature (23), viscosity of muscles and resistance of the vascular bed decrease with heating, hemoglobin and myoglobin link up with more oxygen, thus oxygen uptake increases (21).

While static stretching has been historically integrated into the warm-up routine, recent research has demonstrated that it can lead to a decrease in force production $(18,37,89,94,116$, 122), power production $(36,38,50)$, running speed (141), reaction and movement time (34), and strength endurance $(180,192)$. Conversely, dynamic stretching generally has been shown to 
improve performance while not eliciting negative effects that static $(17,93,94)$ and PNF stretching produce $(55,134)$.

According to Issurin (115), a warm-up should include a general warm-up that includes basic exercises in order to raise blood circulation, increase body circulation and facilitate the oxidative processes in working muscles. After completing this, a special warm-up should follow that includes specially selected drills that activate the coordination mechanisms that are involved in the technical skills to prevent failure in these coordinated skills and thus prevent injuries. With this information, a warm-up for a vertical jump protocol would include a basic dynamic warm-up followed by movements that mimic the vertical jump (188). Since this type of warmup is used in the field, it would be important to use this in a validity and reliability study. In addition, keeping the warm-up constant would be vital to eliminate changes in interday reliability.

\subsubsection{The arm-swing effect}

Countermovement jumps can be performed with arm swing, hands on hips, or with hands resting on a weightless bar across the back. Jumps that include an arm swing have been shown to contribute to $8-14 \%$ of the jumping height and thus give a more positive effect on the outcome $(55,88,94)$. Lees et al. (88) performed a study with college-age (age $=19.9 \pm 3.9$ years) males $(n=20)$. The subjects performed 3 countermovement jumps with and without armswing on a force platform. They also attached 3D markers and took electromyographical recordings. The results show that countermovement jump was increased with arm-swing and was due to increased height (28\%) and velocity (72\%) of the COM at take-off.

The increased height at take-off was due to the elevation of the arm segments and the increased velocity of take-off stemmed from a complex series of events which allowed the arms to build up energy from the shoulder, elbow and hip joints early in the jump and transfer it to the 
rest of the body during the later stages of the jump. This energy was used to increase several mechanisms. First, the energy increased the kinetic and potential energy of the arms at take-off. Second, the energy was stored and released from the muscles and tendons around the ankle, knee and hip joint. Lastly, the energy was used to pull on the body through upward force acting on the trunk at the shoulder. Since there is a large difference in how much arm swing plays a role in jump height, it could potentially be problematic for a validity and reliability study attempting to remove all extraneous variables. Therefore, the hands on hip or hands on a weightless bar method may be beneficial for a study including training status as a variable since the trained population would have a higher level of upper body strength. 


\subsection{Methods and Tools Measuring Human Force-time Curve Variables}

When examining the scientific literature, numerous methods have been performed for quantifying jump height and force-time curve variables during the vertical jump $(73,112,158)$. There are six common electronic methods used to measure jump height: single or multiple linear position transducers (154), force platforms $(31,41,123)$, combinations of linear position transducers and a force platform (31), accelerometers (123), switch mats (31, 41, 67), and optoelectronical systems $(30,120)$.

\subsubsection{Kinematic Methods}

One common method used in the calculation of force-time curve variables is the use of single $(13,14,30,31,44,49,171)$ or multiple linear position transducers $(92)$. The use of the linear position transducer method alone appears to over-estimate power output as a result of an increasing force output because the acceleration component of the force equation is not directly measured but rather dividing the directly measured velocity by time. Thus, suggesting that it is not the best method for accurately assessing vertical jump performance when used as the sole quantification method. Nonetheless, linear position transducers are more cost effective than force platforms. Generally, linear position transducers cost around $\$ 1,000$ or less, while the cost of a force platform is usually at least $\$ 20,000(91)$.

Either one or two linear position transducers can be used to measure jump height. If one linear position transducer is used, kinetic and kinematic variables are calculated from displacement data through double differentiation processes. Bar displacements are measured using one linear position transducer mounted directly above the subject. The linear position transducer produces a voltage signal that must then be collected using some form of analog-todigital conversion and is representative of the degree at which the linear position transducer is extended allowing for displacement-time data to be calculated (90). If a bar is placed on the 
subject's back, it is assumed that the body and the bar move together as a unit; thus, it is assumed that the bar velocity calculated is equivalent to the body velocity.

Instantaneous vertical velocity is calculated from displacement and time data at each sample using the process of double differentiation, which yields the following equation (95):

where $\mathrm{v}=$ instantaneous vertical velocity, $x=$ change in displacement, and $=$ change in time Acceleration is then calculated using a second-order derivative of the displacement data to yield the following equation (31):

where a acceleration, $x=$ change in displacement, and $t=$ change in time .

With this method, double differentiation of displacement data is used to calculate acceleration by tracking the rate of change of velocity between two consecutive points $(31,47)$, which is then coupled with the system mass to calculate a force output (31). Force (F) produced during the lift is determined by adding the acceleration of the system and acceleration resulting from gravity and then multiplying the total acceleration to the mass of the system to yield the following equation:

$$
F=\left(m_{s y s t e m}\right) \cdot\left(a+a_{g}\right)
$$

where $\mathrm{F}=$ force output, $\mathrm{a}=$ acceleration, $\mathrm{a}_{\mathrm{g}}=$ acceleration due to gravity 
Following these calculations, power is determined by multiplying force and velocity at each time point (31).

where $\mathrm{P}=$ power, $\mathrm{F}=$ force, and $\mathrm{v}=$ velocity.

This force output is then multiplied by the velocity-time curve, which is also created from the displacement data. This yields a power-time curve for the movement being analyzed. An outline of the single linear position transducer method can be seen in Figure 2.1.

Utilizing two linear position transducers allows for both vertical and horizontal movements to be measured and can be used to determine the vertical displacement of the bar (185). The linear position transducers are mounted above the subject anteriorly and posteriorly forming a triangle when attached to the barbell (187). To analyze the data, the known displacements are combined with the displacement measurements from the two linear position transducers in the equation and vertical displacement can be calculated. The two linear position transducers and the bar form a triangle in which the dimensions of the triangle are known, permitting both vertical and horizontal movements to be included in the calculation of vertical velocity (31). The same procedures for calculating velocity, acceleration, force, and power using the 1-linear position transducer are used in the displacement data derived from 2-linear position transducers. A summary of the mathematical procedures of the 2-linear position transducer method can be seen in Figure 2.2.

Cormie et al. (47) compared the 1-linear position transducer and 2-linear position transducer methods against a force platform +2 -linear position transducer system in the jump squat. Since a $0 \%$ load jump squat is essentially the same as a countermovement jump, accurate comparisons can be made between the two. The power output produced by both the 1-linear position transducer method and the 2-linear position transducer method were not significantly 
Figure 2.1: A mathematical outline of the LPT method

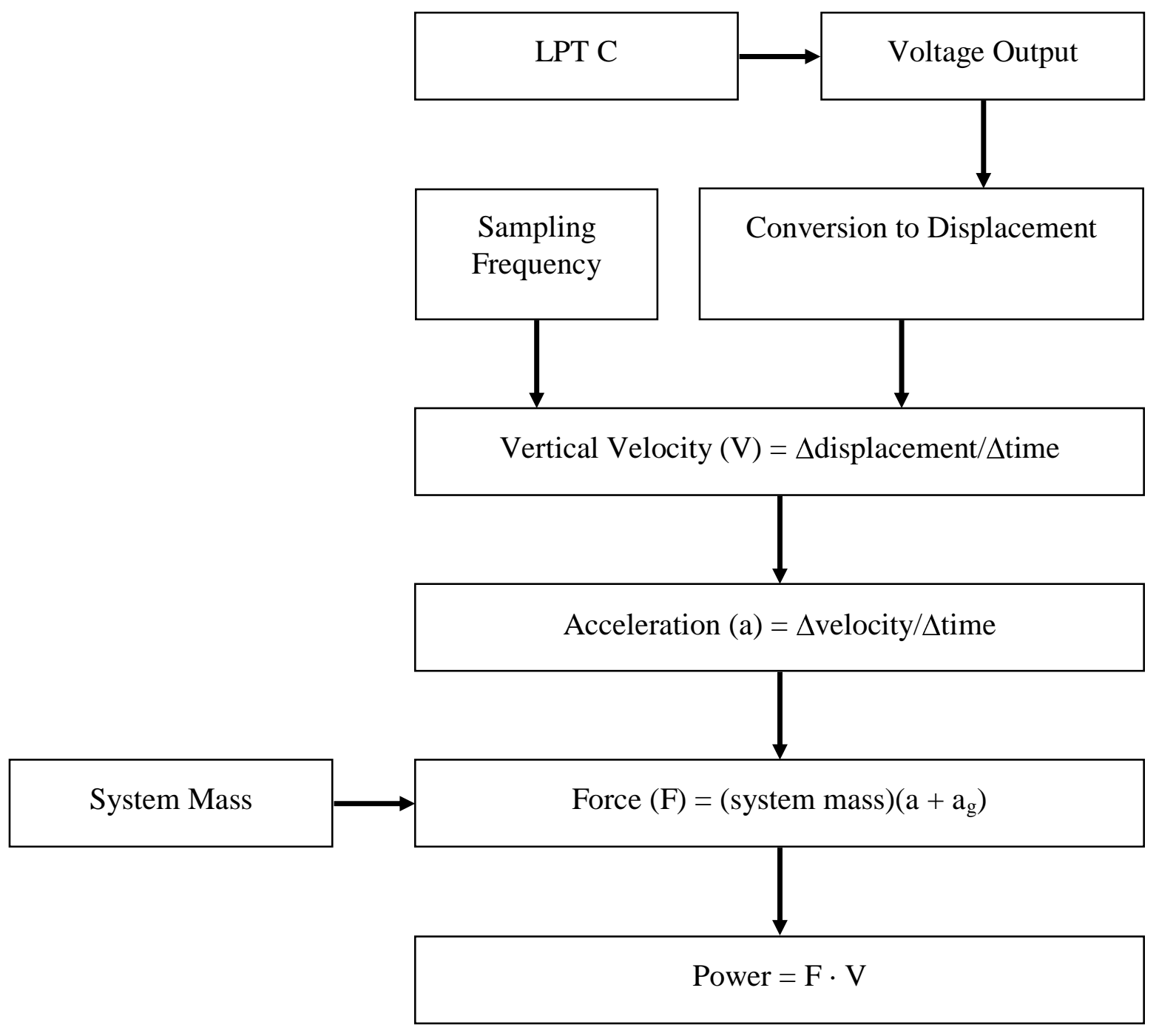


Figure 2.2: A mathematical outline of the 2-LPT method

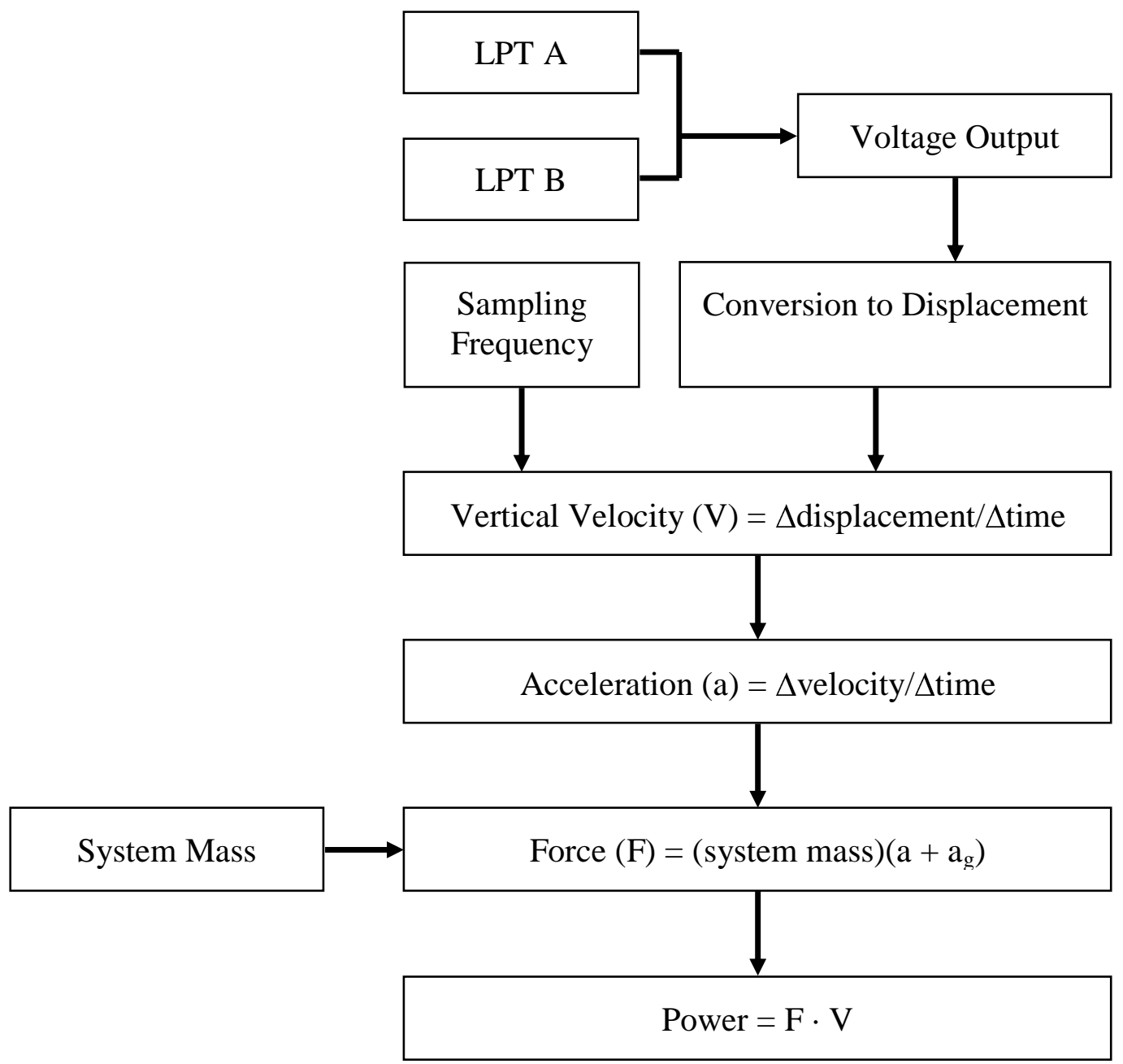


different than the power output produced by the force platform +2 -linear position transducer system.

Cronin et al. (31) tested the validity and reliability of the linear position transducer as a method of measuring jump performance by comparing the mean force, peak force, and time-topeak force measurements with data obtained simultaneously with a force platform in 25 college age males $(23.4 \pm 4.6)$. Mean force, peak force, and time-to-peak produced by the linear position transducer were tested against a force platform. The Pearson correlation coefficients across the 3 jumps for the mean force $(\mathrm{r}=0.95-0.96)$, peak force $(\mathrm{r}=0.86-0.93)$, and time-to-peak force $(\mathrm{r}=$ 0.92-1.0) were high, providing evidence that the linear position transducer and force platform measurements were similar.

Atkinson et al. (31) state that if a high $(\mathrm{r}>0.80)$ and statistically significant correlation coefficient is obtained between the 2 devices, the equipment is deemed to be sufficiently valid. This was the case for the linear position transducer as a method to measure force variables in the countermovement jump. Additionally, the intraday reliability of the jumps measured by the linear position transducer gave an intraclass correlation coefficient of $0.92-0.98$ for mean force, 0.98-0.98 for peak force, and 0.72-0.96 for time-to-peak force. Furthermore, the coefficients of variation were $2.1-4.5 \%$ for mean force, $2.5-8.4 \%$ for peak force, and $4.1-11.8 \%$ for time-topeak force. Thus, the calculations derived from the linear position transducer were very similar to those of the force platform, which provides evidence of the validity of this method for measuring force. Moreover, the data from the linear position transducer were also shown to be reliable as shown by the high ICCs, low CVs, absence of any statistical difference among trials, and similar values noted with the force platform. Therefore, based on these results, the authors suggest that the linear position transducer is a cost-effective, versatile, valid, and reliable means 
for the measurement of force.

\subsubsection{Kinetic Methods}

A second method for determining power output is based upon vertical ground reaction forces, which can be measured on a force platform. Dynamic movements such as the vertical jump are typically monitored with this device $(31,39,67,123,124,151)$. The force platform is then used to generate a force-time curve (41). Generally, a force platform is composed of two square or rectangular rigid structures, which are positioned parallel to the ground one above the other (57). The inferior structure is steady on the ground, while the superior structure is a lightly mobile platform where the subject stands (86). The two structures are linked by four force recorders positioned at each corner of the force platform (151). These force recorders are piezoelectric or strain gauge transducers, which transform the mechanical action of the subject on the platform into an electric signal (67).

This method of analyzing power is based upon impulse, and can be measured because the initial vertical velocity of the system is always zero (30), which is shown in the following equation (30):

where $\mathrm{F}=$ vertical ground reaction forces, $\mathrm{SM}=$ mass of the system, $\mathrm{i}=$ time point, and $\mathrm{a}=$ acceleration.

Acceleration due to gravity is then subtracted from the calculated acceleration data to ensure that only the acceleration produced by the subject is used to determine velocity (30). Instantaneous vertical velocity of the system's COM is determined by the product of acceleration and time data at each data point and is shown in the following equation (30): 
where $\Delta a=a_{(i-1)}-\mathrm{a}_{(i)}$ and $\Delta t=t_{(i-1)}-t_{(i)}$

where $\Delta t=$ change in time and $\Delta a=$ change in acceleration

The derived velocity data is then combined with the original force values in order to calculate the power output of each jump (31).

$$
\mathrm{P}=\mathrm{F} \text {. }
$$

where $\mathrm{p}=$ power, $\mathrm{F}=$ Force, and $\mathrm{v}=$ velocity

Force platforms can also be used to calculate jump height from creating a force-time curve and analyzing the flight time. Flight time is equal to the time when takeoff occurs until the time when landing occurs. However, this can result in an overestimation of jump height due to incorrect landing techniques (31). The incorrect landing technique is due to the ankle and knee joints being fully extended at takeoff, however they are slightly flexed at landing, which causes the subject's COM to be lower (31). A summary of the mathematical methods of the force platform can be seen in Figure 2.3.

Force platforms commonly sample at $1000 \mathrm{~Hz}(30,31,100)$. Recently, Hori et al. (30) examined the reliability and validity of commonly used performance measurements derived from ground reaction force (GRF)-time data during countermovement jumps and the influence of sampling at different frequencies. Twenty-four college age (25.0 \pm 4.4 years) males performed two countermovement jumps on a force platform. The sampling frequency was set at $500 \mathrm{~Hz}$ as a reference and the data were then re-sampled at 400, 250, 200, 100, 50 and $25 \mathrm{~Hz}$ by interpolating between points to assemble a series of data sets corresponding to these frequencies. They found that peak power, force, and velocity measurements derived from GRF were highly reliable $(\mathrm{ICC}=0.92-0.98, \mathrm{CV}=1.3-4.1)$ across the entire range of frequencies; however, peak rate of force development and time to peak power did not meet minimum acceptable ICC at several sampling frequencies. They observed a breakpoint in accuracy at less than $200 \mathrm{~Hz}$ where 
Figure 2.3: A mathematical outline of the force platform method

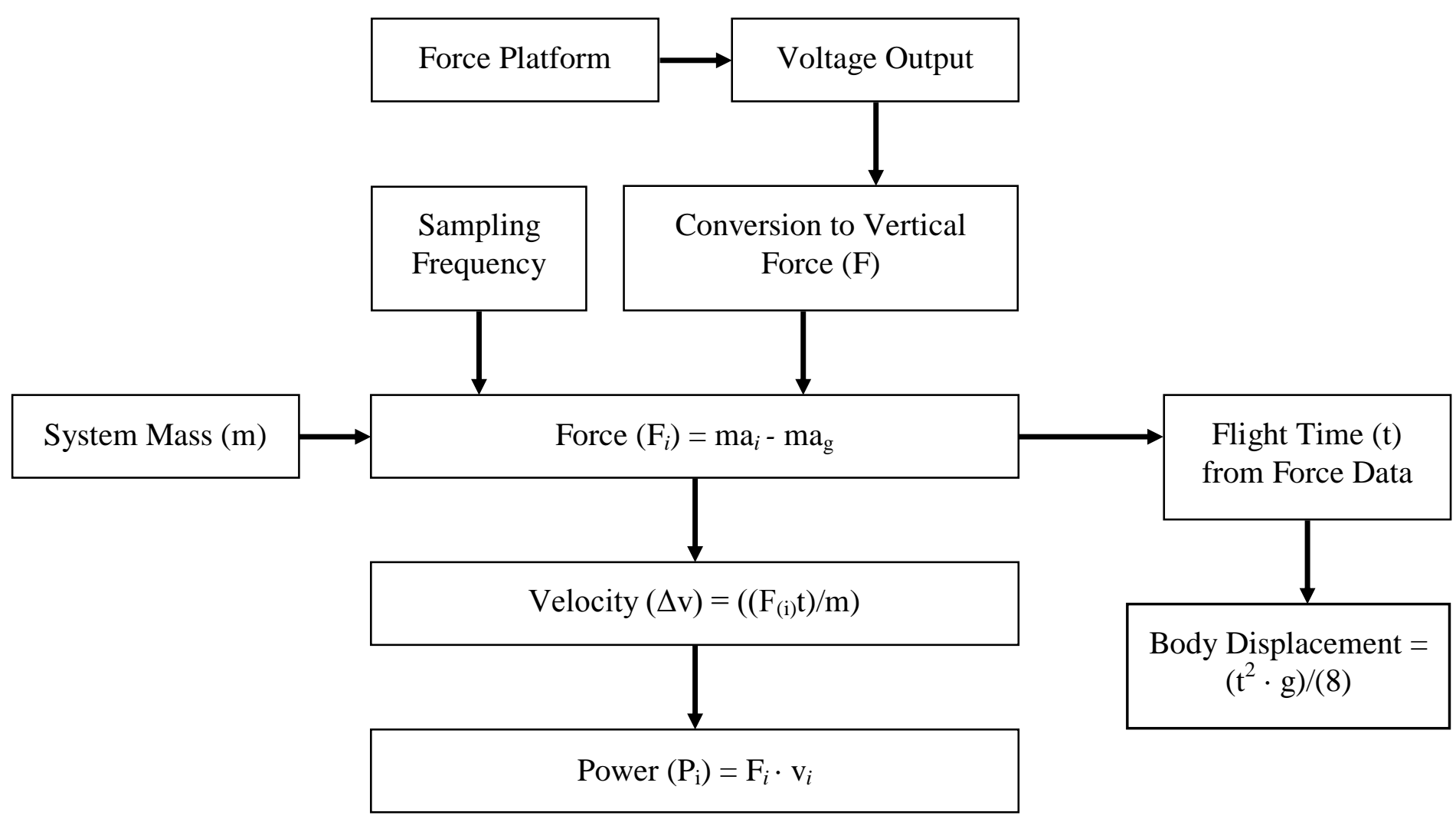


percentage differences increased in most of the measurements. At $200 \mathrm{~Hz}$, ranges in percentage differences were less than $\pm 2 \%$ in all measurements.

Hori et al. (62) also stated that when force is applied toward the force platform, it is apparent that the GRF can vary over time. The subject will continue to apply force throughout their time in contact with the force platform; however, GRF is recorded only at the time points determined by sampling frequency (i.e. every $0.002 \mathrm{~s}$ if sampling frequency is $500 \mathrm{~Hz})(10)$. Thus, a continuously varying phenomenon is being measured at discrete time points with the assumption that change between successive samples is linear (99). If changes in force are too rapid to record at the given sampling frequency, the changes in force occurring between two consecutive samples will not be accurately represented (81). Thus, the rapid change in force could be missed when GRF was sampled at lower frequencies (i.e. longer duration between two time points sampled), such as 25 or $50 \mathrm{~Hz}(81)$.

There is another potential problem with using a force platform as a methodology for estimating power output in the countermovement jump. The technique relies on inverse dynamics to calculate velocity from GRF, which requires extensive data manipulation (81). Similar to kinematic methods reliant on double differentiation, noise amplification and the subsequent risk of erroneous data restricts the force platform's ability to accurately assess power output (81). Cormie et al. (81) provide data to support this mentality. As previously stated, they tested 10 Division I male athletes and found the force platform only method to underestimate peak power by $\sim 1000 \mathrm{~W}$, which was significantly different from the force platform +2 -linear position transducer method (81). 
Hori et al. (31) measured peak rate of force development and time to peak force to examine whether there was any influence of reducing sampling frequencies on shape of the force-time curve. The reliability of peak rate of force development did not meet the minimum acceptable ICC even obtained from $500 \mathrm{~Hz}$. The rapid force development in countermovement jump is produced during the eccentric phase, and a good jumper can keep exerting high force rapidly (187). Therefore, peak rate of force development may appear during the eccentric phase for some athletes, and during the concentric phase for others, depending on each subject's jump technique (e.g. how rapidly and how deep he/she squats during the eccentric phase, how much force he/she generates during concentric phase). This inconsistency in the protocol may be responsible for the low reliability and high standard deviation of peak rate of force development. Normally, peak rate of force development is determined during a static jump, which is a concentric only vertical jump and could minimize these reliability issues. Additionally, sampling at $1000 \mathrm{~Hz}$ could allow for better tracking of rate of force development due to the increased number of samples. 


\subsubsection{Kinematic + Kinetic Methods}

A third method currently used to assess power output is by way of a force platform synchronized with two linear position transducers. If one linear position transducer is used with a force platform, a combination of displacement data and vertical ground reaction forces is used for calculation of power. Displacement data are collected via a single linear position transducer while a force platform measures the vertical force output of the system. The velocity of the system is determined using a first-order derivative of the displacement data. Power is calculated from the product of the velocity and force data. In this instance, force data obtained from the force platform is multiplied by the velocity data obtained from the linear position transducer (31). A mathematical outline of the linear position transducer + force platform method can be seen in Figure 2.4.

If two linear position transducers are used in conjunction with the force platform (Figure 2.5), signals from each linear position transducer are combined to determine vertical displacement, which is subsequently used in combination with time to calculate velocity. Power is then calculated by coupling this velocity with force data collected through the use of a force platform. The benefit of using two linear position transducers is that they account for horizontal movement, which may affect the vertical displacement measured during dynamic multidimensional movements. The two linear position transducers and the bar form a triangle in which the dimensions of the triangle are known, permitting both vertical and horizontal movements to be included in the calculation of vertical velocity. The system is advantageous to the single linear position transducer technique because it tracks displacement in the vertical and the horizontal planes, allowing for a more accurate calculation of power (31). Velocity is then calculated by combining signals from each linear position transducer to determine vertical displacement with time (81). Power is then calculated by coupling this velocity with force data 
Figure 2.4: A mathematical outline of the force platform + single LPT method

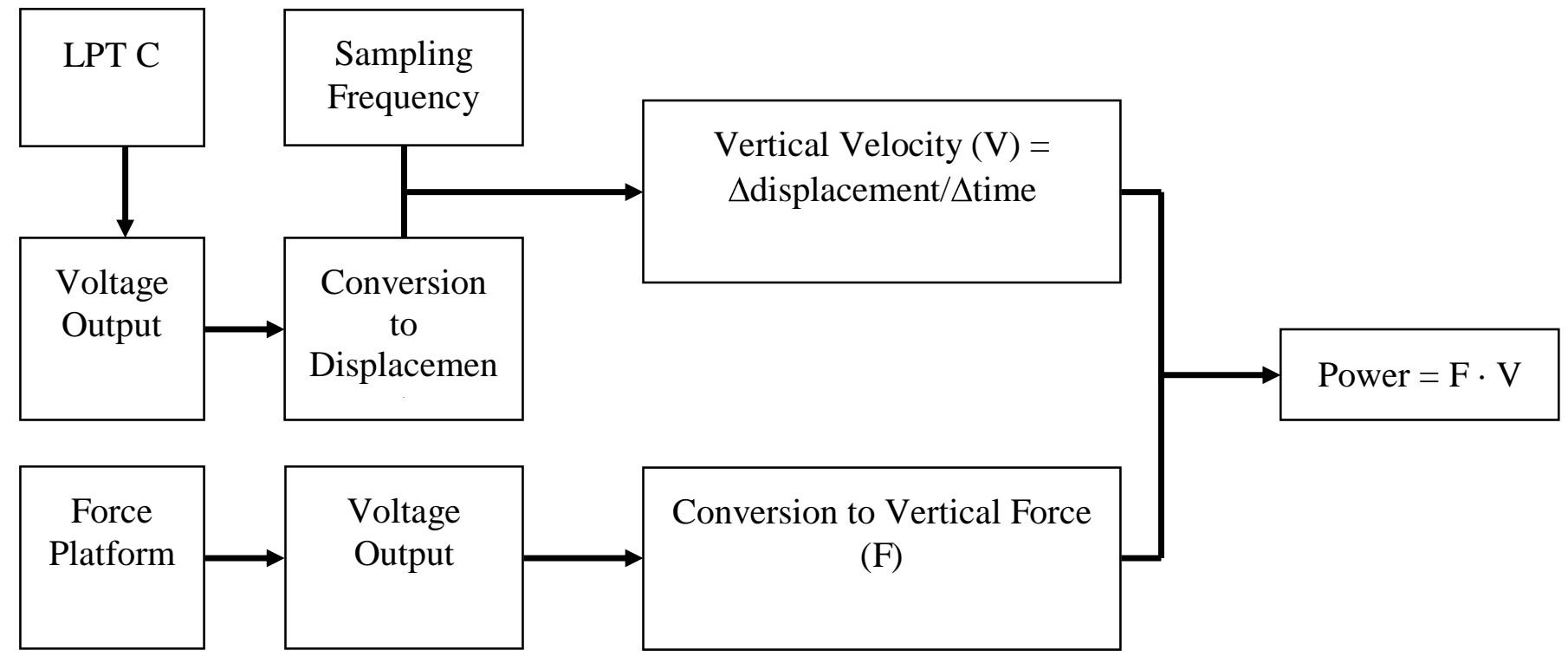


Figure 2.5: A mathematical outline of the force platform + 2-LPT method

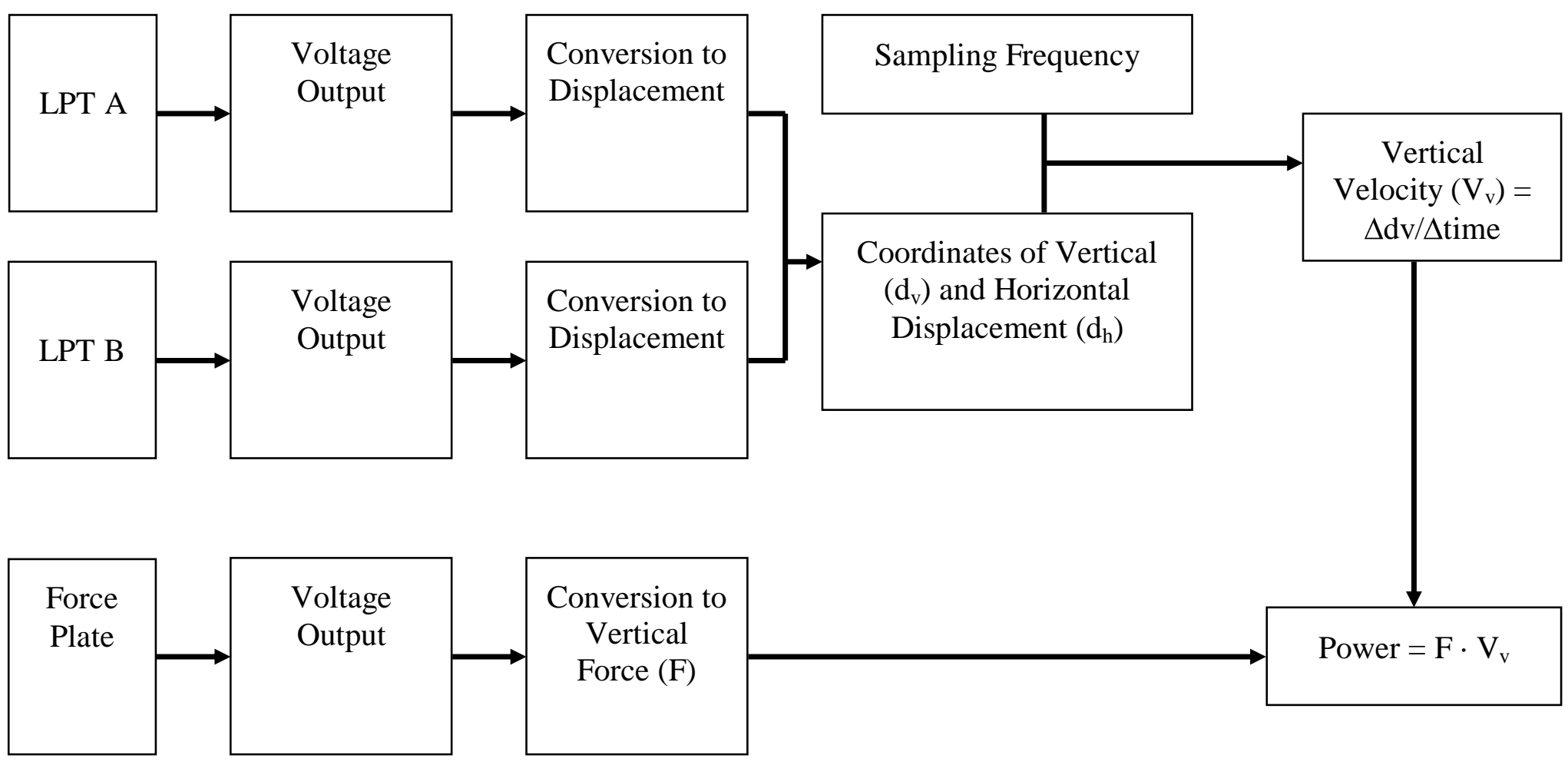


collected through the use of a force platform (143).

This type of methodology has been used in vertical jump testing (194). Additionally, this methodology has been used to test the jump squat, squat, and hang clean. Cormie et al. (31) hypothesized that the use of two linear position transducers and a force platform represent a more accurate measure of displacement, velocity, and power in multidimensional movements. The reliability of this method in the jump squat at $0 \%$ of 1-RM with ten division I male athletes has been reported to be highly reliable $(\mathrm{ICC}=0.95)$. Therefore, this method may be the best comparison point when attempting to determine the validity of accelerometer based testing devices.

\subsubsection{Contact Time Methods}

\subsubsection{Switch Mats}

Switch mats are often considered by strength \& conditioning coaches because flight-timebased measurements have been reported to have a small error of measurement when compared with video analysis (31). In addition, they are relatively inexpensive as the cost of switch-mat measurement systems (without the laptop) are typically less than $\$ 500.00$ (31). Switch mat systems use a basic kinematic equation to calculate jump height from flight time (31). The microswitches embedded in the mat $(0.6858$ by $0.6858 \mathrm{~m})$ time the interval between subject takeoff from the mat and their landing (120). The switch mat contains bars placed longitudinally above one another. These bars form an electric circuit that is closed when a load on the mat causes the bars to press together. The circuit opens as soon as the subject leaves the mat. A digital timer connected to the mat starts recording when the electric circuit opens and stops when it closes (106). Similar to the force platform, the sampling frequency of the digital timer is usually around $1000 \mathrm{~Hz}(7,29,87)$. A mathematical outline of the switch mat system can be seen in Figure 2.6. 
Figure 2.6: A mathematical outline of an optoelectronical/switch mat-based method

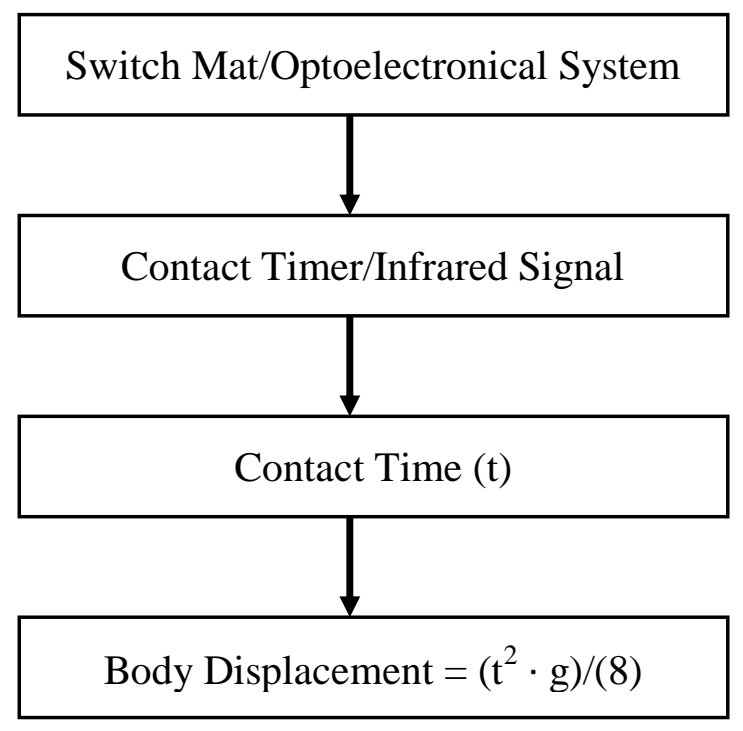


Switch mats are most often interfaced with a laptop computer that records flight time and determines the height of the jump (30). Switch mats can calculate maximal height (in), flight time (milliseconds), and maximum power $\left(\mathrm{P}_{\max }\right)$ estimation (30). They use the following formula (120):

where gravity $=g=9.81 \mathrm{~m} \mathrm{x} \mathrm{s}^{-2}$ and $t=$ flight time

Isaacs et al. (44) and Leard et al. (29) provide data supporting the higher accuracy of timing systems versus jump and reach devices such as the Vertec. Additionally, Moir et al. (100, 110) found that intersession systematic bias was not present when using the highest jump recorded from a contact mat during each testing session in physically active men and women. Furthermore, the validity of switch mats was examined by Garcia-Lopez et al. (87). There was a 6.9 $\pm 3.7 \mathrm{~ms}$ difference in contact times between a switch mat and a force platform sampling at $500 \mathrm{~Hz}$. Also, a $9.2 \pm 1.3 \mathrm{~ms}$ difference in flight times comparing the two systems was determined. The authors state that differences in the switch mat and force platform can be attributed to body mass differences and differences in flight time. The correlation of flight time between the switch mat and force platform was $\mathrm{r}=0.97, \mathrm{p}<0.001$ and the correlation of contact times between the two was $r=0.99, \mathrm{p}<0.001$. The authors suggest the switch mat system overestimates flight time and underestimates contact time. In turn, when calculating power output from prediction equations, this overestimation could further lead to a distortion of power output. 


\subsubsection{Optoelectronical Systems}

Optoelectronical systems are a way of assessing power output by infrared technology.

These systems use infrared signals that are triggered by the feet of the subject at the instant of take-off and on landing to measure the jump height on the basis of flight time $(111,131)$.

Similar to the force platform, optoelectronical systems record data with a sampling frequency of $1000 \mathrm{~Hz}$. This method consists of two connected bars $(100 \times 4 \times 3 \mathrm{~cm})$, which send each other photoelectric signals. One bar is involved in the sending and reception of signals, while the other in their transmission. The two bars are placed parallel on the ground at a distance of 1 meter to each other and are connected to a computer with specific analysis software that can display the data immediately (130). When a body stands between the two bars, all the infrared signals cannot be transmitted and received. Similar to switch mats, a digital timer is connected to the cells and recording starts when the last part of the subject's body leaves the ground, and consequently all the infrared signals can be transmitted and received by the two bars (62). Optoelectronical systems record flight time and calculate jump performance with the flight time method (49).

The validity and reliability of optoelectronical systems were examined by Glatthorn et al. (171) and showed optoelectronical systems to be valid and reliable when compared to a force platform. However, they systematically underestimated the body displacement by $1.5 \mathrm{cms}$. This is due to the fact that the photocells are elevated from the ground, which causes the recorded flight time to be reduced.

\subsubsection{Acceleration-based Methods}

A fourth method for assessing power output in jumping activities is the use of accelerometers (30). Accelerometers measure acceleration using a transducer that outputs a voltage proportional to the gravitational acceleration $\left(\mathrm{a}_{\mathrm{g}}\right)$ plus any linear acceleration the body is 
experiencing (30). This signal is recorded by an analog-to-digital system and computer software (30). Most accelerometers contain a three-axis accelerometer, which means they are capable of detecting either movement or the pull of gravity in three-dimensional space (64).

Very little data exist in the scientific literature on the reliability and validity of accelerometers as measurement tools in jumping activities. In the scientific literature, the accelerometer has been shown to be very comparable to the linear position transducer methods (95). An example of a force-time curve graph derived from acceleration data is shown in Figure 7 and a mathematical outline of the acceleration-based method is shown in Figure 8.

When calculating force-time curve variables from acceleration data, force is the first variable to be calculated. Force can be calculated simply by multiplying the acceleration at any given time point by the mass of the body (47):

$$
\mathrm{F}_{(\mathrm{i})}=\mathrm{ma}_{(\mathrm{i})}-\mathrm{ma}_{(\mathrm{g})}
$$

where $\mathrm{F}=$ force, $\mathrm{m}=$ mass of system, $\mathrm{i}=$ time point, $\mathrm{a}=$ acceleration, and $\mathrm{a}_{\mathrm{g}}=$ acceleration due to gravity

Velocity data are derived by single integration of the acceleration data with respect to time (47):

$$
\mathrm{v}_{(\mathrm{i})}=
$$

where $\mathrm{v}=$ velocity and $\mathrm{t}=1 /$ sampling frequency

Power can then be calculated based on the force and velocity calculated from the previous two equations (119):

$$
\mathrm{P}_{(\mathrm{i})}=\mathrm{F}_{(\mathrm{t})} \cdot \mathrm{V}_{(\mathrm{i})}
$$

where $\mathrm{P}=$ power, $\mathrm{F}=$ force, $\mathrm{v}=$ velocity, $\mathrm{t}=1 /$ sampling frequency, and $\mathrm{i}=$ time point 
Figure 2.7: Force-time Curve Variables Derived from Acceleration Data

Force-time Curve Variables Derived from Acceleration Data

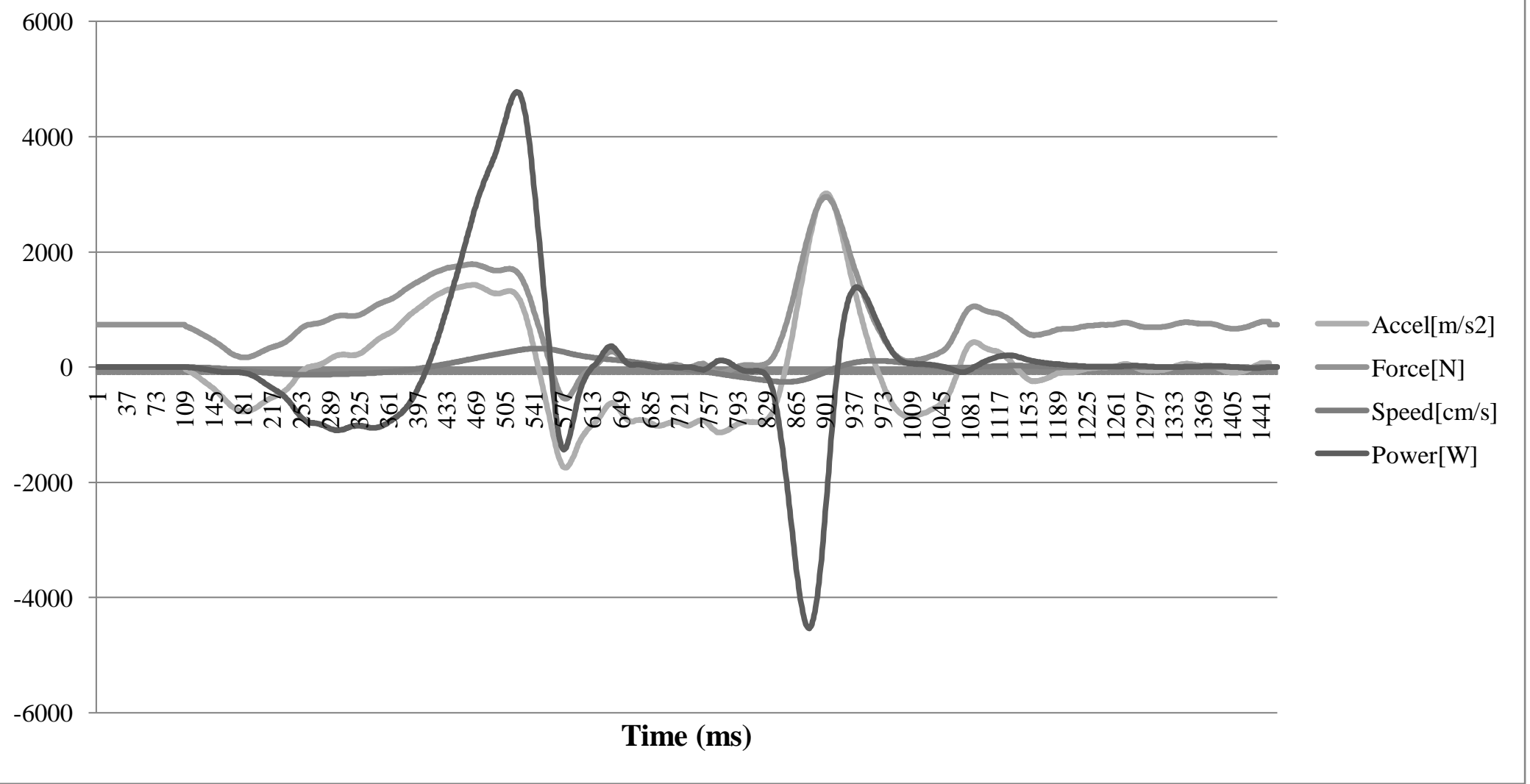


Figure 2.8: A mathematical outline of the acceleration-based method

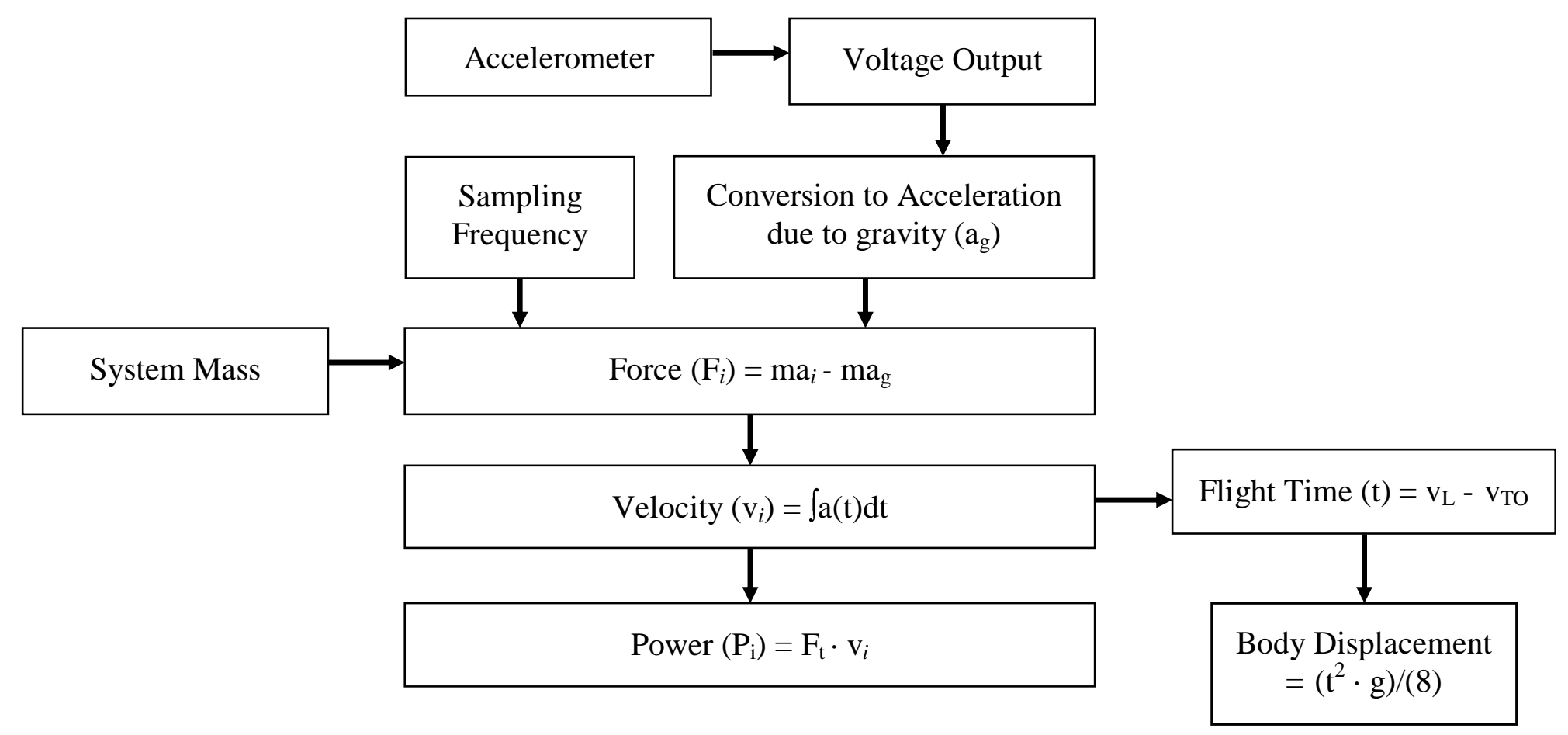


Previously, accelerometers used the vertical takeoff velocity method to measure the jump height.

Recently, the maximal positive vertical velocity $v_{\max }$ replaced the vertical takeoff velocity (95):

where $y_{\mathrm{p}}=$ the position of the subject's COM at the peak of the jump, $\mathrm{v}_{\max }=$ maximal positive vertical velocity, and $g=$ verticlal acceleration due to gravity

The new method involves the use of the flight time method (47):

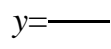

where $\mathrm{FT}=$ flight time $=$ the time interval between the maximal positive and maximal negative velocity of the body during the vertical jump and $g=$ verticlal acceleration due to gravity.

This equation is derived from the following equation:

$$
v_{\mathrm{L}}=v_{\mathrm{TO}}-g \mathrm{t}
$$

where $v_{\mathrm{L}}=$ vertical velocity at landing position, $v_{\mathrm{TO}}=$ vertical velocity at take off, $g=$ vertical acceleration due to gravity, and $t=$ time between landing and takeoff

Since one can assume the subject's landing COM to be equal to its takeoff COM, the equation can be reduced:

$$
\mathrm{v}_{\mathrm{L}}=-\mathrm{v}_{\mathrm{TO}}
$$

The time between takeoff and landing position is included as the flight time (FT):

$$
\mathrm{v}_{\mathrm{L}}=\mathrm{v}_{\mathrm{TO}}-g F T
$$

Substituting $-\mathrm{v}_{\text {TO }}$ for $\mathrm{v}_{\mathrm{L}}$ yields the following equation:

$$
-2 v_{\mathrm{TO}}=-\mathrm{g} F T
$$

and can be reduced to the following equation:

Then this equation can be substituted into the equation for vertical velocity: 
and reduced to:

There are a few potential problems with the accelerometer method. First, the use of accelerometry has the disadvantage that the signal must be integrated to derive velocity data, and this can be error prone (47). In addition, the axis of the accelerometer must remain aligned with the plane of movement, or the acceleration will not be accurately measured (47). Lastly, because of their construction, the appropriate accelerometers are somewhat delicate and could possibly be damaged by any shock that would occur if they were dropped or impacted (30).

To the author's knowledge, there have only been two studies performed examining validity and reliability of the accelerometer to date $(30,47)$. The first study tested vertical acceleration on the bench press. The accelerometer exhibited valid and reliable data until $70 \% 1$ RM. Above 70\% 1-RM, the movement became slower, which caused the accelerometer's data to lose validity and reliability. The second study tested the accelerometer's validity and reliability during vertical jump. The results of this study suggest that accelerometers do not accurately measure the absolute value of the acceleration and consequently the velocity. Thus, this methodology based on flight time provides valid and reliable data. While this study proved validity and reliability by way of flight time, a more accurate way to test flight time is by way of a force platform.

The available data suggest that accelerometers produce reliable velocity and power data in both the back squat and bench press (47). Recently, our laboratory produced pilot data for accelerometers that sample at $200 \mathrm{~Hz}$. The accelerometers were shown to be reliable in the following parameters: vertical jump displacement $[\mathrm{CV} \%=3.1$ (Confidence Interval = 2.4-4.7; ICC $=0.98$ (Confidence Interval $=0.95-0.99)]$, velocity $[C V \%=5.7($ Confidence Interval $=4.5-$ 
9.0); ICC $=0.80($ Confidence $=0.57-0.92)]$, and force output $[\mathrm{CV} \%=2.9($ Confidence Interval $=$ 2.2-4.4); ICC $=0.98$ (Confidence $=0.96-0.99)]$. However, the power outputs determined by this technology demonstrates a very poor reliability $[\mathrm{CV} \%=21.3($ Confidence Interval $=18-38.7)$; ICC $=0.49$ (Confidence Interval $=0.14-0.75)$. Additionally, when the accelerometer data $(200$ $\mathrm{Hz}$ ) were compared to force platform data, which samples at $1000 \mathrm{~Hz}$, velocity, displacement, and power were all significantly different. One potential reason for this lack of validity may be related to the fact that the force platform sampled at $1000 \mathrm{~Hz}$ and the accelerometer used in the pilot work sampled at $200 \mathrm{~Hz}$. Based upon the fact that Hori et al. (47) displayed a large drop off in percentage difference of the force platform once the sampling frequency dropped below 200 $\mathrm{Hz}$, further research is warranted on the effect of lowering sampling frequency in accelerometers in order to determine the cutoff sampling frequency required to maintain reliability. 


\subsubsection{Summary of Methods and Tools Measuring Human Force-time Curve Variables}

Switch mat and accelerometer systems that use flight time to assess jump height are

beneficial because the test is simple and quick to perform, very portable, and cost effective. The optoelectronical method is also simple and quick to perform, but is slightly less portable and cost effective. These systems, in addition to the force platform only system, are useful because arm swing is not required, so the test can focus on lower body power. Additionally, they are not reliant on the subject timing their jump to touch something at the peak of their jump as in a jump and reach method such as a Vertec. However, the subject must land on the mat with their legs almost fully extended to produce a valid result. If the legs are bent when landing, an overestimation of jump height will result. All of the aforementioned methods may be slightly lower than methods with something to reach for at the top for motivation.

Each of these techniques are based on valid but different mathematical premise; however, in each case described in this thesis, the data are manipulated, differentiated, or integrated, which amplifies any noise in the raw signal (30). The increased data manipulation leads to a greater risk of accumulating error in the results and reduces the validity and reliability of the calculated power output (95). However, this is to a much lesser degree in the force platform + linear position transducer method because it can measure force and displacement directly where the other methods must differentiate or integrate one of these variables (95). In addition to the disadvantage of excessive data manipulation, the kinetic-only technique requires at least 1 point within the data where velocity is zero (81). This is necessary to use the impulse-momentum approach, which is very sensitive to this condition (47). Therefore, as previously stated, the force platform + linear position transducer is considered the gold standard for estimating forcetime curve variables in the countermovement jump. Thus, when determining the validity of accelerometers, it would be warranted to compare the results to the force platform + linear 
position transducer device.

\subsection{Validity \& Reliability}

\subsubsection{Validity}

Validity is defined as the extent to which a method test measures what it is supposed to measure. Globally, there are three type of validity: internal, external, and prediction validity (47). Internal validity refers to how well the tool measures the variable in question (47).

External validity concerns the ability of the tool to predict changes in a population other than the one being studied (47). Prediction validity refers to the ability to predict one variable from another (47). Sensitivity is also a part of validity. Sensitivity of measurement is the degree that small differences can be detected. More sensitive instruments allow greater precision of measurement and so enhance validity (168). There are several different types of validity used in exercise physiology research that can be classified as either logical or statistical validity (168).

Logical validity is a relatively weak type of validity that doesn't use statistics or numerical values to express the degree of accuracy of a test or instrument. Logical validity is further divided into face and content validity. Face validity, the weakest type of validity, simply says that the device is measuring what it's supposed to be measuring by way of look. For example, the vertical jump test being used to measure jump height would have high face validity. The other type of logical validity, content validity, is the extent to which the items or questions accurately measure the desired information, such as on a questionnaire (168).

Conversely, statistical validity is considered to be stronger than logical validity because it has a numerical value, which can be compared to a standard value. Statistical validity can then be broken down into criterion-based validity, concurrent validity, and predictive validity. Criterion-based validity is the degree to which scores on a test are related to some recognized standard or criterion (168). Concurrent validity includes two measures of the same variable 
obtained within a close period. A criterion test is applied in addition to a test or instrument to be validated. From these two tests, accuracy can be determined by the degree of statistical relationship between the two measures. Another type of statistical validity, predictive validity, uses a test or instrument to predict the occurrence of some future event (19). Lastly, construct validity is used when the variable of interest, the construct variable, has no definitive criterion, is difficult to measure, or cannot be directly observed. This reflects the ability of a method to measure an abstract concept (19). Furthermore, reliability constitutes an integral part of validity. A test cannot be considered valid if it is not reliable (19).

\subsubsection{Reliability}

Reliability can be defined as the consistency or repeatability of test scores or data (173).

It means that repeated measurements of the same variable under the same conditions should give similar scores. Reliability gives an index for the amount of measurement error. Whether a method can be considered as reliable or not depends on the acceptability of the measurement error for practical use (19). Unlike validity, a method can be reliable without being valid. This is the reason why the reliability of a method should be tested before its validity in a validation study (173). Test-retest reliability has to do with the degree to which an instrument can produce the same measurements at different times under the same conditions (173). To provide an accurate assessment of reliability, the effects of learning should be removed to minimize systematic error $(9,19)$. Familiarization sessions should be performed to ensure performance changes are not the result of learning effects (9).

There are four different measurement error sources: the participant, the testing, the scoring and the instrumentation (168). The errors that are caused by the participants are due to fluctuation in mood and motivation, to fatigue and to familiarization with the test protocol between the repeated tests. The errors that are due to testing are caused by differences in the 
management of the protocol and/or in the instruction of the participants between the tests; thus, strengthening the need for standardization of the methods. Scoring measurement errors are produced by incompetence or inexperience of the tester during the evaluation of the measurements.

There are then measurement errors due to the instrumentation or instrumentation measurement methodology. Methods of establishing reliability include intraclass correlation (ICC) and the coefficient of variance (CV) (79). For researchers interested in retest correlation as a measure of reliability, the ICC derived from a mixed model is unbiased for any sample size (80). The ICC is typically a ratio of the variance of interest over the sum of the variance of interest plus error (130). According to Hopkins (173), use of the intraclass correlation is also the only sensible approach to computing an average correlation between more than two trials. In addition, the $\mathrm{CV}$ is a normalized measure of dispersion or a probability distribution. The $\mathrm{CV}$ is expressed in the following equation (168):

where $=$ standard deviation and = mean

\subsubsection{Sensitivity}

Sensitivity of measurement is the degree that small differences can be detected. More sensitive instruments allow greater precision of measurement and so enhance validity (168). More sensitivity is usually better when dealing with accelerometers. For example, for a given change in acceleration, there will be a larger change in signal and since larger signal changes are easier to measure, you will get more accurate readings. Accelerometers have been shown to have a sensitivity of $99 \%$ (168). However, this measure may be affected by sampling rate as the change in acceleration may be more difficult to read as fewer samples per second are collected. 


\subsection{Summary and Implications of Literature Review}

The assessment and development of athletes' capability of power output is an important research topic among sports scientists and practitioners $(19,51,168)$. The power output during the vertical jump is often considered to represent athletic potential because it indicates underlying leg extensor qualities due to the specificity of muscle groups involved, types of muscle actions, range of motion, and pattern of movements $(19,81)$. Although Hori(136) examined the reliability and validity of mechanical quantities (i.e. power, velocity and force over time) during the vertical jump movement on a force platform, these variables need to be thoroughly examined with accelerometer technology. Furthermore, the influence of the frequencies at which data are sampled has not been examined outside of Hori's work; thus, further research is warranted in this area.

While the importance of power output is widely accepted, not all strength \& conditioning coaches have access to expensive, immovable equipment such as a force platform or linear position transducer (135). Thus, it is paramount to examine the validity and reliability of measurements that can be easily administered in the strength \& conditioning facility (81). The two "field test" methods mentioned in this thesis are the switch mat and accelerometer. The switch mat has been shown to be valid and reliable against a force platform; however, the accelerometer provides additional data about force-time curve variables that the switch mat cannot give. The vertical jump test is an excellent indicator of power output $(81,137)$ and thus playing ability (81). Therefore, accelerometers can give strength \& conditioning coaches very useful information for monitoring training progression and indicating potential sports performance if proven valid and reliable. 
To prove these systems valid and reliable, the study's protocol must be standardized in the amount of familiarization time, warm-up, time-of-day, arm swing, self-talk, and rest interval. A familiarization session should be included to prevent a learning curve for novice, untrained subjects and to become familiarized with the laboratory setting and electronic devices used in the study. The warm-up should be dynamic in nature and include activities that emulate the vertical jump. Additionally, for multiple vertical jumps, the rest interval between warm-up and test sets should be close to three minutes. Furthermore, the subjects must come at the same time-of-day each session to prevent diurnal effects. Arm-swing should be eliminated to examine strictly the lower body power and eliminate differences in subjects with a higher level of upper body strength. The self-talk should be instructional in nature and allow the athlete to reach their highest capable vertical jump, such as one they would produce in strength \& conditioning settings. 


\section{REFERENCES}

1. Aagaard P, Andersen JL, Dyhre-Poulsen P, Leffers AM, Wagner A, Magnusson SP, Halkjaer-Kristensen J, Simonsen EB. A mechanism for increased contractile strength of human pennate muscle in response to strength training: changes in muscle architecture. J Physiol. 2001;534(Pt. 2):613-23.

2. Aagaard P, Simonsen EB, Andersen JL, Magnusson P, Dyhre-Poulsen P. Increased rate of force development and neural drive of human skeletal muscle following resistance training. J Appl Physiol. 2002;93(4):1318-26.

3. Aagaard P, Simonsen EB, Andersen JL, Magnusson SP, Halkjaer-Kristensen J, DyhrePoulsen P. Neural inhibition during maximal eccentric and concentric quadriceps contraction: effects of resistance training. J Appl Physiol. 2000;89(6):2249-57.

4. Ahtiainen JP, Pakarinen A, Alen M, Kraemer WJ, Hakkinen K. Muscle hypertrophy, hormonal adaptations and strength development during strength training in strength-trained and untrained men. Eur J Appl Physiol. 2003;89(6):555-63.

5. Anderson L, Aagaard P. Influence of maximal muscle strength and intrinsic muscle contractile properties on contractile rate of force development. Eur J Appl Physiol. 2006;96(1):46-52.

6. Aragon-Vargas LF. Evaluation of Four Vertical Jump Tests: Methodology, reliablility, validity, and accuracy. Meas Phys Educ Exerc Sci. 2000;4(4):215-28.

7. Asmussen E, Bonde-Petersen F. Storage of elastic energy in skeletal muscles in man. Acta Physiol Scand. 1974;91(3):385-92.

8. Atkinson G, Nevill AM. Statistical methods for assessing measurement error (reliability) in variables relevant to sports medicine. Sports Med. 1998;26(4):217-38. 
9. Baca A. A comparison of methods for analyzing drop jump performance. Med Sci Sports Exerc. 1999;31(3):437-442.

10. Baker D. Improving vertical jump performance through general, special, and specific strength training: a brief review. J Strength Cond Res. 1996;10(2):131-6.

11. Baker D. Differences in strength and power among junior- high, senior-high, college-aged, and elite professional rugby league players. J Strength Cond Res. 2002;16(4):581-5.

12. Baker D, Nance $\mathrm{S}$. The relation between running speed and measures of strength and power in professional rugby league players. J Strength Cond Res. 1999;13(3):230-5.

13. Baker D, Nance S, Moore $M$. The load that maximizes the average mechanical power output during jump squats in power-trained athletes. J Strength Cond Res. 2001;15(1):92-7.

14. Barfield JP, Sells PD, Rowe DA, Hanningan K. Practice effect of the wingate anaerobic test. J Strength Cond Res. 2002;16(3):472-273.

15. Barnes JL. Relationship of jumping and agility performance in female volleyball athletes. $J$ Strength Cond Res. 2007;21(4):1192-6.

16. Behm D. Effect of acute static stretching on force, balance, reaction time, and movement time. Med Sci Sports Exerc. 2004;36(8):1397-402.

17. Behm DG, Button DC, Butt JC. Factors affecting force loss with prolonged stretching. Can J Appl Physiol. 2001;26(3):261-72.

18. Berg KE and Latin RW. Essentials of Reserach Methods in Health, Physical Education, Exercise Science, and Recreation. Philadelphia (PA): Lippincott Williams \& Wilkins; 2008, 300 p. 
19. Bergh U, Thorstensson A, Sjodin B, Hulten B, Piehl K, Karlsson J. Maximal oxygen uptake and muscle fiber types in trained and untrained humans. Med Sci Sports Exerc. $1978 ; 10(3): 151-4$.

20. Bernard T. Time-of-day effects in maximal anaerobic leg exercise. Eur J Appl Physiol Occup Physiol. 1998;77(1-2):133-8.

21. Bobbert MF, Van Soest AJ. Effects of muscle strengthening on vertical jump height: a simulation study. Med Sci Sports Exerc. 1994;26(8):1012-1020.

22. Bompa TO, Haff GG. Periodization: Theory and Methodology of Training. 5th ed. Champaign (IL): Human Kinetics Publishers; 2009, 384 p.

23. Bosco C, Tarkka I, Komi P. Effect of elastic energy and myoelectrical potentiation of triceps surae during stretch-shortening cycle exercise. Int J Sports Med. 1982;3(3):137-40.

24. Bottinelli R, Canepari M, Pellegrino MA, Reggiani C. Force-velocity properties of human skeletal muscle fibres: myosin heavy chain isoform and temperature dependence. $J$ Physiol. 1996;495( Pt 2):573-86.

25. Bret C. Leg strength and stiffness as ability factors in $100 \mathrm{~m}$ sprinting. J Sports Med Phys Fitness. 2002;42(3):274-81.

26. Campos GE, Luecke TJ, Wendeln HK, Toma K, Hagerman FC, Murray TF, Ragg KE, Ratamess NA, Kraemer WJ, Staron RS. Muscular adaptations in response to three different resistance-training regimens: specificity of repetition maximum training zones. Eur J Appl Physiol. 2002;88(1-2):50-60.

27. Canavan PK, Garret GE, Armstrong LE. Kinematic and kinetic relationships between an olympic-style lift and the vertical jump. J Strength Cond Res. 1996;10(2):127-30. 
28. Carlock J, Smith A, Hartman M, Morris R, Ciroslan D, Pierce KC, Newton RU, Stone MH. The relationship between vertical jump power estimates and weightlifting ability: a field test approach. J Strength Cond Res. 2004;18(3):534-9.

29. Casartelli N. Validity and reliability of the Myotest measurement system assessing vertical jump performance [thesis]. Zurich (CH): ETH Zurich; 2009. 62 p.

30. Cormie P, McBride JM, McCaulley GO. Validation of power measurement techniques in dynamic lower body resistance exercises. J Appl Biomech. 2007;23(2):103-18.

31. Cormie P, McBride JM, McCaulley GO. Power-time, force-time, and velocity-time curve analysis during the jump squat: impact of load. J Appl Biomech. 2008;24(2):112-20.

32. Cormie P, McCaulley GO, McBride JM. Power versus strength-power jump squat training: influence on the load-power relationship. Med Sci Sports Exerc. 2007;39(6):996-1003.

33. Cornwell A. Acute effects of stretching on the neuromechanical properties of the triceps surae muscle complex. Eur J Appl Physiol. 2002;86(5):428-34.

34. Costill DL, Miller SJ, Myers WC, Kehoe FM, Hoffman WH. Relationship among selected tests of explosive leg strength and power. Res $Q .1968 ; 39(3): 785-7$.

35. Cramer JT, Housh TJ, Johnson GO, Miller JM, Coburn JW, Beck TW. Acute effects of static stretching on peak torque in women. J Strength Cond Res. 2004;18(2):236-41.

36. Cramer JT, Housh TJ, Weir JP, Johnson GO, Coburn JW, Beck TW. The acute effects of static stretching on peak torque, mean power output, electromyography, and mechanomyography. Eur J Appl Physiol. 2005;93(5-6):530-9.

37. Cramer JT, Housh TJ, Coburn JW, Beck TW, Johnson GO. Acute effects of static stretching on maximal eccentric torque production in women. $J$ Strength Cond Res. 2006;20(2):354-8. 
38. Cronin JB, Hing RD, McNair PJ. Reliability and validity of a linear position transducer for measuring jump performance. J Strength Cond Res. 2004;18(3):590-3.

39. Cronin JB, Hansen KT. Strength and power predictors of sports speed. J Strength Cond Res. 2005;19(2):349-57.

40. Delecluse C, Roelants M, Diels R, Koninckx E, Verschueren S. Effects of whole body vibration training on muscle strength and sprint performance in sprint-trained athletes. Int $J$ Sports Med. 2005;26(8):662-8.

41. Deschenes M. Short review: rate coding and motor unit recruitment patterns. J Appl Sports Sci Res. 1989;3(2):33-9.

42. Desmedt JE, Godaux E. Ballistic contractions in man: characteristic recruitment pattern of single motor units of the tibialis anterior muscle. J Physiol (Lond). 1977;264(3):673-93.

43. Ditroilo $\mathrm{M}$, Fernàndez $\mathrm{PE}$, Benelli $\mathrm{P}$. The measurement of vertical jump performance. Coach \& Sport Sci J. 2005;1:26-30.

44. Dowling JJ, Vamos L. Identification of kinetic and temporal factors related to vertical jump performance. J Appl Biomech. 1993;9:95-110.

45. Duchateau J, Semmler JG, Enoka RM. Training adaptations in the behavior of human motor units. J Appl Physiol. 2006;101(6):1766-75.

46. Dugan EL, Doyle TL, Humphries B, Hasson CJ, Newton RU. Determining the optimal load for jump squats: a review of methods and calculations. J Strength Cond Res. 2004;18(3):668-74.

47. Enoka RM. Muscle strength and its development: new perspective. Sports Med. 1988;6:146-68. 
48. Erculj F. Impact of fatigue on the position of the release arm and shoulder girdle over a longer shooter distance for an elite basketball player. J Strength Cond Res. 2009;23(3):1029-36.

49. Evetovich TK. Impact of prior exercise on hamstring flexibility: a comparison of proprioceptive neuromuscular facilitation and static stretching. J Strength Cond Res. 2003;17(3):484-8.

50. Faber M. Applicability of the coefficient of variation method for analyzing synaptic plasticity. Biophys J. 1991;60(5):1288-94.

51. Flanagan EP. Reliability of the reactive strength index and time to stabilization during depth jumps. J Strength Cond Res. 2008;22(5):1677-82.

52. Fleck S, Kraemer WJ. Designing Resistance Training Programs. 3rd ed. Champaign (IL): Human Kinetics; 2004, 375 p.

53. Fleck SJ, Kraemer WJ. Resistance training: physiological responses and adaptation (part 3 of 4). Phys Sportsmed. 1988;16(5):63-6;72;4;6.

54. Fletcher IM, Jones B. The effect of different warm-up stretch protocols on 20 meter sprint performance in trained rugby union players. J Strength Cond Res. 2004;18(4):885-8.

55. Folland JP, Williams AG. The adaptations to strength training: morphological and neurological contributions to increased strength. Sports Med. 2007;37(2):145-68.

56. French DN, Gomez AL, Volek JS, Rubin MR, Ratamess NA, Sharman MJ. Longitudinal tracking of muscular power changes of NCAA Division I collegiate women gymnasts. $J$ Strength Cond Res. 2005;18(1):101-7.

57. Fry AC. The role of resistance exercise intensity on muscle fibre adaptations. Sports Med. 2004;34(10):663-79. 
58. Fry AC, Schilling BK, Staron RS, Hagerman FC, Hikida RS, Thrush JT. Muscle fiber characteristics and performance correlates of male olympic-style weightlifters. J Strength Cond Res. 2003;17(4):746-54.

59. Fry AC, Webber JM, Weiss LW, Harber MP, Vaczi M, Pattison NA. Muscle fiber characteristics of competitive power lifters. J Strength Cond Res. 2003;17(2):402-10.

60. Gabriel DA, Kamen G, Frost G. Neural adaptations to resistive exercise: mechanisms and recommendations for training practices. Sports Med. 2006;36(2):133-49.

61. Garcia-Lopez D. The validation of a new method that measures contact and flight times during vertical jump. Intl J Sport Physiol. 2005;26(4): 294-302

62. Garhammer J. Equipment for the development of athletic strength and power. Nat Strength Coaches Assoc J. 1981;3(6):24-6.

63. Glatthorn JF, Gouge S, Impellizzeri FI, Maffiuletti NA. Validity and reliability of optojump photoelectric cells for measuring vertical jump height. J Sci Med Sport. (in press).

64. Gollhofer A. Behavior of triceps surae muscle-tendon complex in different jump conditions. Eur J Appl Physiol. 1992;64(4):283-91.

65. Green HB. Regulation of fiber size, oxidative potential, capillarization in human muscle by resistance exercise. Am J Physiol. 1998;276(45):591-6.

66. Haff GG, Stone MH, O'Bryant HS, Harman E, Dinan CN, Johnson R, Han KH. Force-time dependent characteristics of dynamic and isometric muscle actions. J Strength Cond Res. 1997;11(4):269-72.

67. Haff GG, Whitley A, Potteiger JA. A brief review: explosive exercises and sports performance. Natl Strength Cond Assoc. 2001;23(3):13-20. 
68. Häkkinen K. Neuromuscular adaptations during strength training, aging, detraining, and immobilization. Crit Rev Physic Rehab Med. 1994;6:161-98.

69. Hakkinen K, Komi P, Kauhanen H. Isometric force and relaxation-time, electromyographic and muscle fiber characteristics of leg extensor muscles. Acta Physiol Scand. $1985 ; 125: 144-51$.

70. Hannie PQ, Hunter G, Kekes-Sabo T, Nicholson C, Harrison PC. The effects of recovery on force production, blood lactate, and work performed during bench press exercise. $J$ Strength Cond Res. 1995;9(1):8-12.

71. Hardy J. Speaking clearly: a critical review of the self-talk literature. Psychol Sport Exerc. 2006;7(1):81-97.

72. Harman EA, Rosenstein MT, Frykman PN, Rosenstein RM. The effects of arms and countermovement on vertical jumping. Med Sci Sports Exerc. 1990;22(6):825-33.

73. Harman EA, Rosenstein MT, Frykman PN, Rosenstein RM, Kraemer W. Estimation of human power output from vertical jump. J Appl Sport Sci Res. 1991;5(3):116-20.

74. Hather BM, Tesch PA, Buchanan P, Dudley GA. Influence of eccentric actions on skeletal muscle adaptations to resistance training. Acta Physiol Scand. 1991;143(2):177-85.

75. Henneman E, Somjen G, Carpenter DO. Excitability and inhibitability of motoneurons of different sizes. J Neurophysiol. 1965;28(3):599-620.

76. Holcomb WR, Lander JE, Rutland RM, Wilson GD. A biomechanical analysis of the vertical jump and three modified plyometric depth jumps. J Strength Cond Res. $1996 ; 10(2): 83-8$. 
77. Holtermann A, Roeleveld K, Engstrom M, Sand T. Enhanced H-reflex with resistance training is related to increased rate of force development. Eur J Appl Physiol. 2007;101(3):301-12.

78. Hopkins WG. Measures of reliability in sports medicine and science. Sports Med. 2000;30(1):1-15.

79. Hopkins WG. Reliability of power in physical performance tests. Sports Med. 2001;31(3):211-34.

80. Hori N. Assessment and enhancement of lower extremity power of athletes. joondalup, western australia [dissertation]. Perth, (AS): Edith Cowan Univeristy; 2007. 185 p.

81. Hori N, Newton RU, Andrews WA, Kawamori N, McGuigan MR, Nosaka K. Comparison of four different methods to measure power output during the hang power clean and the weighted jump squat. J Strength Cond Res. 2007;21(2):314-20.

82. Hori N, Newton RU, Nosaka K, McGuigan MR. Comparison of different methods of determining power output in weightlifting exercises. Strength and Cond J. 2006;28(2):3440.

83. Houston ME, Froese EA, Valeriote SP, Green HJ, Ranney DA. Muscle performance, morphology and metabolic capacity during strength training and detraining: a one leg model. Eur J Appl Physiol. 1983;51(1):25-35.

84. Ikai M, Fukunaga T. A study of training effect on strength per unit cross sectional area of muscle by means of ultrasonic measurement. Inter Z Angew Physiol. 1970;28(3):173-80.

85. Iossifidou A, Baltzopoulos V, Giakas G. Isokinetic knee extension and vertical jumping: are they related? J Sports Sci. 2005;23(10):1121-7. 
86. Isaacs SD. Comparison of the vertec and just jump systems for measuring height of vertical jump by young children. Percept Mot Skills. 1998;86(2):659-63.

87. Issurin V. Block periodization versus traditional training theory: a review. J Sports Med Phys Fitness. 2008;48(1):65-75.

88. Issurin V. Block Periodization: Breakthrough in Sports Training. Michigan (US): Ultimate Athlete Concepts; 2008, 213 p.

89. Izquierdo M, Hakkinen K, Gonzalez-Badillo JJ, Ibanez J, Gorostiaga EM. Effects of longterm training specificity on maximal strength and power of the upper and lower extremities in athletes from different sports. Eur J Appl Physiol. 2002;87(3):264-71.

90. Izquierdo M, Hakkinen K, Ibanez J, Garrues M, Anton A, Zuniga A, Larrion JL, Gorostiaga EM. Effects of strength training on muscle power and serum hormones in middle-aged and older men. J Appl Physiol. 2001;90(4):1497-507.

91. Izquierdo M, Ibanez J, Gorostiaga E, Garrues M, Zuniga A, Anton A, Larrion JL, Hakkinen K. Maximal strength and power characteristics in isometric and dynamic actions of the upper and lower extremities in middle-aged and older men. Acta Physiol Scand. 1999;167(1):57-68.

92. Jackson AS, Pollock ML. Generalized equations for predicting body density of men. $B r J$ Nutr. 1978;40(3):497-504.

93. Jeffreys I. Warm-up and Stretching. In: Baechle TR, Earle RW, editors. Essentials of Strength Training and Conditioning. Champaign (IL): Human Kinetics Publishers; 2008, pp. 296-76. 
94. Jidvotseff B, Croisier JL, Lhermerout C, Serre L, Sac D, Crielaard JM. The concept of isoinertial assessment: reproducibility analysis and descriptive data. Isokin Exerc Sci. 2006;14(1):53-62.

95. Johnson DL, Bahamonde R. Power output estimate in university athletes. J Strength Cond Res. 1996;10(3):161-6.

96. Kamen $\mathrm{G}$. The acquisition of maximal isometric plantar flexor strength: a force time curve analysis. J Motor Behav. 1983;15(1):63-73.

97. Kawamori N, Rossi SJ, Justice BD, Haff EE, Pistilli EE, O'Bryant HS, Stone MH, Haff GG. Peak force and rate of force development during isometric and dynamic mid-thigh clean pulls performed at various intensities. J Strength Cond Res. 2006;20(3):483-91.

98. Kibele A. Possibilities and limitations in the biomechanical analysis of countermovement jumps: a methodological study. J Applied Biomech. 1998;14(1):105-17.

99. Klavora P. Vertical-jump tests: a critical review. Strength and Cond J. 2000;22(5):70-5.

100. Komi P. Signal Characteristics of EMG at different levels of muscle tension. Acta Physioligica Scandinavica. 1976;96(2):267-76.

101. Komi PV. How important is neural drive for strength and power development in human skeletal muscle? In: Saltin B, editor. Biochemistry VI. Champaign (IL): Human Kinetics; 1986, pp. 515-29.

102. Komi PV. Training of muscle strength and power: interaction of neuromotoric, hypertrophic, mechanical factors. Int J Sports Med. 1986;7(Suppl 1):10-5.

103. Komi PV. Stretch-shortening cycle: a powerful model to study normal and fatigued muscle. J Biomech. 2000;33(10):1197-206. 
104. Komi PV. Strength and Power In Sport. 2nd ed. Malden (MA): Blackwell Scientific; 2003, $523 \mathrm{p}$.

105. Komi PV, Bosco C. Utilization of stored elastic energy in leg extensor muscles by men and women. Med Sci Sports Exerc. 1978;10(4):261-5.

106. Kraemer WJ, Patton JF, Gordon SE, Harman EA, Deschenes MR, Reynolds K, Newton RU, Triplett NT, Dziados JE. Compatibility of high-intensity strength and endurance training on hormonal and skeletal muscle adaptations. J Appl Physiol. 1995;78(3):976-89.

107. Kraemer WJ, Volek JS. Creatine supplementation. Its role in human performance. Clin Sports Med. 1999;18(3):651-66, ix.

108. Kraska JM, Ramsey MJ, Haff G, Fethke N, Sands WA, Stone ME, Stone MH. Relationship between strength characteristsics and unweighted and weighted vertical jump. Int J Sport Physiol Perf. 2009;4(4):461-73

109. Lamont HS. Effects of 6 weeks of periodized squat training with or without whole-body vibration on short-term adaptations in jump performance within recreationally resistance trained men. J Strength Cond Res. 2008;22(6):1882-93.

110. Leard J. Validity of two alternative systems for measuring vertical jump height. J Strength Cond Res. 2007;21(4):1296-9.

111. Lees A, Vanrenterghem J, De Clercq DJ. Understanding how an arm swing enhances performance in the vertical jump. J Biomech. 2004;37(12):1929-40.

112. Lieber RL. Skeletal Muscle Structure, Function, \& Plasticity: The Physiological Basis of Rehabilitation. Baltimore (MD): Lippincott Williams \& Wilkins; 2002. 448 p.

113. Liebermann DG, Katz L. On the assessment of lower-limb muscular power capability. Isokin Exerc Sci. 2003;11(2):87-94. 
114. Little T, Williams AG. Effects of differential stretching protocols during warm-ups on high-speed motor capacities in professional soccer players. J Strength Cond Res. 2006;20(1):203-7.

115. Lundeen WA, Hayes DK, Pauly JE, Reiter RJ. Chronobiology: Its Role in Clinical Medicine, General Biology and Agriculture. In: Proceedings of the Xix International Conference of the International Society for Chronobiology; 1990 Jun 20-24: Bethesda, (MD).

116. MacDougall JD, Ward GR, Sale DG, Sutton JR. Biochemical adaptation of human skeletal muscle to heavy resistance training and immobilization. J Appl Physiol. 1977;43(4):700-3.

117. Major JA, Sands WA, McNeal JR, Paine DD, Kipp R. Design, construction, and validation of a portable one-dimensional force platform. J Strength Cond Res. 1998;12(1):37-41.

118. Mark D, LaMarche J. Beginning iPhone development: exploring the iPhone SDK. Berkeley (CA): Apress; 2008, 584 p.

119. Markovic G, Dizdar D, Juckic I, Cardinale M. Reliability and factorial validity of squat and countermovement jump tests. J Strength Cond Res. 2004;18(3):551-5.

120. Maughan R. Relationship between muscle strength and muscle cross-sectional area. Implications for training. Sports Med. 1984;1(4):263-9.

121. Mcardle WD, Katch FI, Katch VL. Exercise Physiology: Energy, Nutrition, and Human Performance. 6th ed. Baltimore (MD): Lippincott, Williams \& Wilkins; 2007, 1067 p.

122. McBride JM, Triplett-McBride T, Davie A, Newton RU. A comparison of strength and power characteristics between power lifters, olympic lifters, and sprinters. J Strength Cond Res. 1999;13(1):58-66. 
123. McBride JM, Triplett-McBride T, Davie A, Newton RU. The effect of heavy- vs. light-load jump squats on the development of strength, power, and speed. J Strength Cond Res. 2002;16(1):75-82.

124. McGinnis PM. Technology in biomechanics. In: Biomechanics of Sport and Exercise. Champaign (IL): Human Kinetics; 2005, pp. 361-70.

125. McGuigan MR, Doyle TL, Newton M, Edwards DJ, Nimphius S, Newton RU. Eccentric utilization ratio: effect of sport and phase of training. J Strength Cond Res. 2006;20(4):9925 .

126. Mero A, Luhtanen P, Viitasalo JT, Komi PV. Relationships between maximal running velocity, muscle fiber characteristics, force production and force relaxation of sprinters. Scand J Sports Sci. 1981;3(1):16-22.

127. Milner-Brown HS, Stein RB, Lee RG. Synchronization of human motor units: possible roles of exercise and supraspinal reflexes. Electroencephalogr Clin Neurophysiol. $1975 ; 38(3): 245-54$.

128. Mirkov DM, Nedeljkovic A, Milanovic S, Jaric S. Muscle strength testing: evaluation of tests of explosive force production. Eur J Appl Physiol. 2004;91(2-3):147-54.

129. Moir G, Sanders R, Button C, Glaister M. The influence of familiarization on the reliability of force variables measured during unloaded and loaded vertical jumps. J Strength Cond Res. 2005;19:140-5.

130. Moir G, Shastri P, Connaboy C. Intersession reliability of vertical jump height in women and men. J Strength Cond Res. 2008;22(6):1779-84.

131. Moritani T, Sherman WM, Shibata M, Matsumoto T, Shinohara M. Oxygen availability and motor unit activity in humans. Eur J Appl Physiol Occup Physiol. 1992;64(6):552-6. 
132. Mortini T, deVries HA. Neural factors versus hypertrophy in the time course of muscle strength gain. Am J Phys Med. 1979;58(3):115-30.

133. Nelson AG, Driscoll NM, Landin DK, Young MA, Schexnayder IC. Acute effects of passive muscle stretching on sprint performance. J Sports Sci. 2005;23(5):449-54.

134. Newton RU. Developing explosive muscular power: implications for a mixed method training strategy. Strength and Cond J. 1994;16(5):20-31.

135. Newton RU, Dugan E. Application of Strength Diagnosis. Strength and Cond J. 2002;24(5):50-9.

136. Newton RU, Hakkinen K, Hakkinen A, McCormick M, Volek J, Kraemer WJ. Mixedmethods resistance training increases power and strength of young and older men. Med Sci Sports Exerc. 2002;34(8):1367-75.

137. Plisk SS, Stone MH. Periodization strategies. Strength and Cond J. 2003;25(6):19-37.

138. Ploutz LL, Tesch PA, Biro RL, Dudley GA. Effect of resistance training on muscle use during exercise. J Appl Physiol. 1994;76(4):1675-81.

139. Ploutz-Snyder LL, Giamis EL. Orientation and familiarization to 1RM strength testing and young women. J Strength Cond Res. 2001;15(4):519-23.

140. Power KE. An acute bout of static stretching: effects on force and jumping performance. Med Sci Sports Exerc. 2004;36(8):1389-96.

141. Read M, Cisar CJ. The influence of varied rest interval lengths on depth jump performance. J Strength Cond Res. 2001;15(3):279-83.

142. Reiser RF, 2nd, Rocheford EC, Armstrong CJ. Building a better understanding of basic mechanical principles through analysis of the vertical jump. Strength and Cond J. 2006;28(4):70-80. 
143. Robinson JM, Stone MH, Johnson RL, Penland CM, Warren BJ, Lewis RD. Effects of different weight training exercise/rest intervals on strength, power, and high intensity exercise endurance. J Strength and Cond Res. 1995;9(4):216-21.

144. Roman WJ. Adaptations in elbow flexors of elderly males after heavy-resistance training. $J$ Appl Physiol. 1993;74(2):750-4.

145. Rotto DM, Kaufman MP. Effect of metabolic products of muscular contraction on discharge of group III and IV afferents. J Appl Physiol. 1988;64(6):2306-13.

146. Ruther C. Hypertrophy, Resistance Training, and the Nature of Skeletal Muscle Activation. J Strength Cond Res. 1995;9(3):155-9.

147. Sahlin K, Ren JM. Relationship of contraction capacity to metabolic changes during recovery from a fatiguing contraction. J Appl Physiol. 1989;67(2):648-54.

148. Sale DG. Effect of strength training upon motorneuron excitability in man. Med Sci Sports Exerc. 1983;15(1):57-62.

149. Sale DG. Neural Adaptations to Strength Training. Med Sci Sport Exerc.1988; 20(5); S13545 .

150. Sands WA, Smith LS, Kivi DM, McNeal JR, Dorman JC, Stone MH. Anthropometric and physical abilities profiles: US national skeleton team. Sport Biomech. 2005;4(2):197-214.

151. Sayers SP, Harackiewicz DV, Harman EA, Frykman PN, Rosenstein MT. Cross-validation of three jump power equations. Med Sci Sports Exerc. 1999;31(4):572-7.

152. Schmidtbleicher D. Training for power events. In: Komi PV, editor. Strength and Power in Sport. Oxford (UK): Blackwell; 1992, pp. 381-5.

153. Semmler JG. Motor unit synchronization and neuromuscular performance. Exerc Sport Sci Rev. 2002;30(1):8-14. 
154. Semmler JG, Kornatz KW, Dinenno DV, Zhou S, Enoka RM. Motor unit synchronisation is enhanced during slow lengthening contractions of a hand muscle. J Physiol. 2002;545(Pt 2):681-95.

155. Siff MC. Biomechanical foundations of Strength and power training. In: V. Zatsiorsky editor. Biomechanics in Sport. London (UK), Blackwell Scientific Ltd; 2001. p. 103-139.

156. Siff MC. Supertraining. 6th ed. Denver (CO): Supertrainig Institute; 2003, 496 p.

157. Slinde F. Test-Retest Reliability of Three Different Countermovement Jumping Tests. $J$ Strength Cond Res. 2008;22(2):640-4.

158. Staron RS. Human skeletal muscle fiber types: delineation, development, and distribution. Can J Appl Physiol. 1997;22(4):307-27.

159. Staron RS, Karapondo DL, Kraemer WJ, Fry AC, Gordon SE, Falkel JE, Hagerman FC, Hikida RS. Skeletal muscle adaptations during early phase of heavy-resistance training in men and women. J Appl Physiol. 1994;76(3):1247-55.

160. Staron RS, Leonardi MJ, Karapondo DL, Malicky ES, Falkel JE, Hagerman FC, Hikida RS. Strength and skeletal muscle adaptations in heavy-resistance-trained women after detraining and retraining. J Appl Physiol. 1991;70(2):631-40.

161. Staron RS, Malicky ES, Leonardi MJ, Falkel JE, Hagerman FC, Dudley GA. Muscle hypertrophy and fast fiber type conversions in heavy resistance-trained women. Eur J Appl Physiol. 1990;60(1):71-9.

162. Stone MH. Literature review: explosive exercises and training. Natl Strength Cond Assoc J. $1993 ; 15(3): 7 ; 9-15$.

163. Stone MH, Johnson RL, Carter DR. A short term comparison of two different methods of resistance training on leg strength and power. J Athl Training. 1979:158-60. 
164. Stone MH, O'Bryant HO. Weight Training: A Scientific Approach. Edina (MN): Burgess; 1987. $364 \mathrm{p}$.

165. Stone MH, O'Bryant HS, McCoy L, Coglianese R, Lehmkuhl M, Schilling B. Power and maximum strength relationships during performance of dynamic and static weighted jumps. J Strength Cond Res. 2003;17(1):140-7.

166. Stone MH, Sanborn K, O'Bryant HS, Hartman M, Stone ME, Proulx C, Ward B, Hruby J. Maximum strength-power-performance relationships in collegiate throwers. J Strength Cond Res. 2003;17(4):739-45.

167. Stone MH, Stone ME, Sands WA. Principles and Practice of Resistance Training. Champaign (IL): Human Kinetics Publishers; 2007, 376 p.

168. Sukop J, Nelson R. Effect of isometric training on the force-time characteristics of muscle contraction. In: RC Nelson and CA Morehouse editors. Biomechanics IV. Baltimore (MD): University Park Press; 1974, p. 440-7.

169. Tesch PA, Karlsson J. Muscle fiber types and size in trained and untrained muscles of elite athletes. J Appl Physiol. 1985;59(6):1716-20.

170. Tessitore A, Meeusen R, Pagano R, Benvenuti C, Tiberi M, Capranica L. Effectiveness of active versus passive recovery strategies after futsal games. J Strength Cond Res. 2008;22(5):1402-12.

171. Theodorakis Y. The effects of motivational versus instructional self-talk on improving motor performance. Sport Psychol. 2000;14:253-72.

172. Thomas JR, Nelson JK, Silverman SJ. Research Methods in Physical Activity. Champaign (IL): Human Kinetics; 2005. 472 p. 
173. Thorstensson A, Karlsson J, Viitasalo JH, Luhtanen P, Komi PV. Effect of strength training on EMG of human skeletal muscle. Acta Physiol Scand. 1976;98(2):232-6.

174. Tod D. Effects of instructional and motivational self-talk on the vertical jump. J Strength Cond Res. 2009;23(1):196-202.

175. Van Cutsem M, Duchateau J, Hainaut K. Changes in single motor unit behaviour contribute to the increase in contraction speed after dynamic training in humans. $J$ Physiol. 1998;513(Pt 1):295-305.

176. Viitasalo JT, Hakkinen K, Komi PV. Isometric and dynamic force production and muscle fiber composition in man. J Human Mov Studies. 1981;7:199-209.

177. Volek J, Kraemer W. Creatine supplementation: its effect on human muscular performance and body composition. J Strength Cond Res. 1993;10:200-10.

178. Waggener GT, Sessoms ED, Barfield WR. Prediction of maximal vertical jump height, revisited. Int Sports J. 2002;6(1):107-11.

179. Wallman H. Surface electromyographic assessment of the effect of static stretching of the gastrocnemius on vertical jump performance. J Strength Cond Res. 2005;19(3):684-8.

180. Walsh M. The Validation of A Portable Force Plate for Measuring Force-Time Data During Jumping and Landing Tasks. J Strength Cond Res. 2006; 20(4):730-4.

181. Walshe AD, Wilson GJ, Murphy AJ. The validity and reliability of a test of lower body musculotendinous stiffness. Eur J Appl Physiol. 1996;73(3-4):332-9.

182. Widrick JJ, Stelzer JE, Shoepe TC, Garner DP. Functional properties of human muscle fibers after short-term resistance exercise training. Am J Physiol Regul Integr Comp Physiol. 2002;283(2):R408-16. 
183. Wilson GJ, Lyttle KJ, Ostrowski KJ, Murphy AJ. Assessing dynamic performance: a comparison of rate of force development tests. J Strength Cond Res. 1995;9(3):176-81.

184. Winter DA. Biomechanics and Motor Control of Human Movement. 2nd ed. New York (NY): John Wiley and Sons, Inc.; 1992. 277 p.

185. Wisløff U, Castagna C, Helgerud J, Jones R, Hoff J. Strong correlation of maximal squat strength with sprint performance and vertical jump height in elite soccer players. $\mathrm{Br} J$ Sports Med. 2004;38(3):285-8.

186. Wood GA. Data smoothing and differentiation procedures in biomechanics. Exercise and Sport Science Reviews. 1982;10:308-62.

187. Yamaguchi T. Effects of static stretching for 30 seconds and dynamic stretching on leg extension power. J Strength Cond Res. 2005;19(3):677-83.

188. Yao W, Fuglevand RJ, Enoka RM. Motor-unit synchronization increases EMG amplitude and decreases force steadiness of simulated contractions. J Neurophysiol. 2000;83(1):44152.

189. Young W. Laboratory strength assessment of athletes. N Stud Athletics. 1995;10(1):89-96.

190. Young W, MacDonald C, Heggen T, Fitzpatrick J. An evaluation of the specificity, validity and reliability of jumping tests. J Sports Med Phys Fitness. 1997;37(4):240-5.

191. Young WB, Behm DG. Effects of running, static stretching and practice jumps on explosive force production and jumping performance. J Sports Med Phys Fitness. 2003;43(1):21-7.

192. Young WB, Bilby GE. The effect of voluntary effort to influence speed of contraction on strength, muscular power, and hypertrophy development. J Strength Cond Res. 1993;7(3):172-8. 
193. Young WB, Newton RU, Doyle TL, Chapman D, Cormack S, Stewart G. Physiological and anthropometric characteristics of starters and non-starters and playing positions in elite australian rules football: a case study. J Sci Med Sport. 2005;8(3):333-45.

194. Zinsser N. Cognitive techniques for building confidence and enhancing performance. In: J. M. Williams, editor. Applied sport psychology: Personal growth to peak performance. 4th ed. Mountain View (CA): McGraw-Hill; 2006. p. 284-311. 


\title{
Chapter 3. Manuscript written in MSSE style of all data
}

\section{Validity and reliability of accelerometers for examining vertical jump performance}

\author{
Ryan M. Ruben ${ }^{1}$; Prue Cormie ${ }^{2}$; Heather Saffel ${ }^{1}$; Jean L. McCrory ${ }^{1}$; Randall W. Bryner ${ }^{1}$; G. \\ Gregory Haff ${ }^{1}$ \\ ${ }^{1}$ Division of Exercise Physiology, West Virginia University School of Medicine, Morgantown, \\ WV 26505 \\ ${ }^{2}$ Edith Cowan University, Perth, AUSTRALIA
}

Running Head: $\quad$ Validity and reliability of accelerometers

\section{Corresponding Author:}

\author{
G. Gregory Haff \\ WVU School of Medicine \\ Division of Exercise Physiology \\ West Virginia University \\ P.O. Box 9227 \\ Morgantown, WV 26505-9227 \\ Phone: 304-293-4299 \\ Fax: 304-293-7105 \\ Email: ghaff@hsc.wvu.edu
}

\section{Disclosure Statement:}

Myotest Inc. donated the accelerometer systems that were used in the performance of this investigation. 


\section{ABSTRACT}

Purpose: The objectives of this study were to determine the validity and reliability of an accelerometer as a device for estimating performance characteristics and to examine the effect of reducing sampling frequency. Methods: Sixty college aged men and women (age=23.6 $\pm 3.1 \mathrm{y}$; height $=180.1 \pm 6.3 \mathrm{~cm}$; weight $=85.0 \pm 15.2 \mathrm{~kg}$; body fat $=14.2 \pm 6.5 \%)$ performed 10 restricted countermovement vertical jumps with no external load each for a total of 600 jumps. Peak force, rate of force development (RFD), peak power output, peak velocity, flight time and peak vertical displacement were assessed with the use of a tri-axial accelerometer and compared to a force platform + linear position transducer system. The data from the accelerometer were then resampled to determine the optimum sampling frequency. Reliability was assessed by intraclass correlation (ICC) and coefficient of variance (CV). Validity was evaluated by a linear regression analyses to determine a calibration equation, the standard error of the estimate (SEE) and a validity correlation coefficient. Results: The accelerometer was found to be reliable for peak force, peak power, peak velocity and peak displacement for each sampling frequency and had the highest validity at $250 \mathrm{~Hz}$. Regardless of the sampling frequency, the accelerometer significantly overestimated peak force, peak RFD, flight time and displacement while it underestimated peak velocity and peak power. Conclusions: The accelerometer lacks the precision of a force platform + linear position transducer system; however, it can be considered a valid and reliable device for measuring peak power, peak force and flight time at frequencies as low as $50 \mathrm{~Hz}$.

Key Words: Performance Testing, Athlete Monitoring, Power, Countermovement Jump 


\section{INTRODUCTION}

Paragraph 1 . The vertical jump test is an easily measured activity that has been observed to have mechanical similarities to weightlifting movements $(4,8,12)$ as well as other athletic activities $(2,28,33)$. For example, the vertical jump has been positively correlated to the clean and jerk (5) and other markers of performance, such as sprinting (8), agility (2), maximal running velocity (28), and maximal strength (33). One of the benefits of the vertical jump test is it can be carried out quickly and with relatively little interference with training (5). The results of such field tests can provide the coach with valuable information about the potential of the athlete, monitor fluctuations in fatigue and preparedness that occur during a periodized training program, and help provide information about the athlete that can be used to guide the training program (5, $35)$.

Paragraph 2. There are various ways to estimate vertical jump height including single or multiple linear position transducers (7), force platforms $(9,14,27)$, combinations of linear position transducers and a force platform (7), accelerometers (6), jump-and-reach methods (20), switch mats (26), and optoelectronical systems $(11,31)$. Although these devices have been shown to be valid and reliable, some are not precise and each has limitations that affect their usefulness. For example, the switch mat, optoelectronical system, and the jump and reach method fail to give information about performance characteristics including force, power, and velocity. In order to quantify these values, an accelerometer, force platform, linear position transducer system or some combination of these methods must be utilized (19). While the force platform and linear position transducer systems give insight into force-time characteristics, they are expensive, hard to transport, and often require a degree of technical skill that most coaches do not posses (25). 
Paragraph 3. Recently, the use of accelerometers to test vertical jump height has become increasingly popular (29). Most accelerometers contain a tri-axial accelerometer that measures acceleration of the load (i.e. body mass) using a transducer that outputs a voltage proportional to the gravitational acceleration $\left(\mathrm{ag}_{\mathrm{g}}\right)$ plus any linear acceleration the body is experiencing in three dimensional space (10). This signal is recorded by an analog-to-digital system and computer software (10), from which performance variables can be calculated. Accelerometers offer several advantages over the force platform and linear position transducer system including increased portability and automated analysis procedures. However, the information given from the accelerometer must be valid and reliable to effectively aid the coach in improving athletic performance $(5,35)$.

Paragraph 4. When testing performance variables with these types of devices, sampling frequency is a critical variable to consider because reducing it can have an impact on the sensitivity and accuracy of the data in high speed movements (19). Generally, expensive hardware, such the force platform or linear position transducer, is required to generate high frequencies and, as a result, generate larger data files and thus need disk storage space (19). Conversely, a device such as an accelerometer with high portability usually possesses a lower sampling frequency (19). This lower sampling frequency allows for more files to be stored on each unit and enables quicker upload times. However, lowering the sampling frequency can also affect the validity and reliability of the device (19). Thus, it is important for the sport scientists and strength and conditioning coaches to consider what would be the minimum required sampling frequency of an accelerometer to accurately measure performance variables during the vertical jump. 
Paragraph 5. Sato et al. (30) observed that accelerometers are highly correlated with acceleration derived from video analysis in a high pull; however, there have been no published studies to the author's knowledge on the validity and reliability of a tri-axial accelerometer for measuring vertical jump performance variables. Currently, a force platform + linear position transducer system is considered to be an effective method for measuring performance variables, such as those seen in the vertical jump test because the force platform directly measures force and the linear position transducers directly measure displacement and velocity (7). However, there is a paucity of research comparing the accelerometer and force platform + linear position transducer methodologies. This lack of scientific support limits the usefulness of accelerometers to sport scientists and practitioners who often need to provide scientific evidence for the instruments they use. Hence, the central purposes of this original investigation are to examine if an accelerometer is a valid and reliable tool for estimating performance characteristics during a countermovement vertical jump test and if so, establish the optimal sampling frequency required for an accelerometer to produce valid, reliable and accurate data.

\section{METHODS}

Paragraph 6. Experimental Design. This original investigation used a within-

participant repeated-measures design. Testing sessions involved one familiarization session and one testing session each separated by seven days. The countermovement jump performance data obtained with an accelerometer was tested against kinetic and kinematic data obtained from a force platform + linear position transducer system as described previously (7).

Paragraph 7. Subjects. 30 men $($ age $=22.1 \pm 2.3$ years, height $=1.80 \pm 0.07 \mathrm{~m}$, mass $=$ $83.3 \pm 13.5 \mathrm{~kg}$, body fat $=15.1 \pm 5.3 \%)$ and 30 women $($ age $=20.3 \pm 1.4$ years, height $=1.70 \pm$ $0.10 \mathrm{~m}$, mass $=64.8 \pm 9.5 \mathrm{~kg}$, body fat $=21.7 \pm 7.1 \%)$ for a total of 60 subjects $($ age $=21.2 \pm$ 
2.1 years, height $=1.75 \pm 0.10 \mathrm{~m}$, mass $=70.0 \pm 14.9 \mathrm{~kg}$, body fat $=18.4 \pm 7.0 \%$ ) with a wide variety of training backgrounds were recruited for participation in this original investigation. Hopkins et al. (16) found that a reasonable precision for estimates of reliability requires approximately 50 study participants and at least 3 trials. Additionally, the subject pool included trained and untrained men and women to determine if the accelerometer is valid and reliable in the whole spectrum of jumping abilities.

Paragraph 8. Subjects were asked to refrain from exercise for 48 hours before each testing session. Additionally, subjects reported to the West Virginia University Instructional Laboratory where they voluntarily read and signed a written informed consent form, a training history questionnaire, and completed a health history questionnaire in accordance with the American College of Sports Medicine Guidelines (32) and the guidelines set by the West Virginia University Institutional Review Board (H-21109) before participating in the study. All tests were performed at the same time of day in order to minimize any diurnal performance effects.

Paragraph 9. Biometric Data. The preliminary testing session was used to determine the subject's height, body mass, and body composition. This information was used to give a descriptive analysis about the subjects' body characteristics. Body mass was measured using a calibrated electronic scale (BOD POD, Concord, CA, USA) to within $0.01 \mathrm{~kg}$. Height was measured to the nearest $0.1 \mathrm{~cm}$ using a stadiometer. Body composition was then assessed with the use of the Bod Pod (BOD POD, Concord, CA, USA). The body density determined by the Bod Pod was then placed into the Siri Equation to determine the subject percent body fat (32). The reliability of the Bod Pod system in our laboratory has consistently produced intraclass correlation coefficient (ICC) values greater than 0.90 . 
Paragraph 10. Warm-up Procedures. The familiarization and testing sessions began with a 5-minute warm-up on a cycle ergometer (Cyclops, Saris, Inc, WI) performed at a cadence of $70 \mathrm{rpm}$ with a resistance that yielded an intensity between 90-100 Watts. Following the cycling portion of the warm-up, each subject performed a standardized 5-minute dynamic warmup that consisted of high-knees, lunges, power skips, side shuffles, inchworms and toe touches $(21,22,29)$. The dynamic movement warm-up was carefully monitored during each session to ensure that the each session was performed in the exact same fashion. The exact same warm-up was performed before each testing session.

Paragraph 11. Performance Testing. After completing the preliminary testing and the dynamic warm-up, the subjects were familiarized with the jumping protocol and the use of an accelerometer. Subjects first performed five countermovement jump vertical jumps. Then, the subjects rested passively for three minutes after which they performed a second set of five countermovement jumps for a total of ten jumps.

Paragraph 12. Data Acquisition and Analysis Procedures. A tri-axial inertial accelerometer (54.2 x 102.5 x $10.7 \mathrm{~mm}$ ) (Myotest Inc., Royal Oaks, MN) sampling at $500 \mathrm{~Hz}$ was used to determine force, power, velocity and jump height. The subjects performed the jumps while holding a PVC pipe $(0 \mathrm{~kg})$ on the base of their neck just below $\mathrm{C} 7$, which the accelerometer was attached to (7). All vertical jump testing was performed with the subjects standing on a force platform (Rice Lake Scales, Fairmont WV) with the left and right side of the PVC pipe each attached to two linear position transducers (Celesco PT5A-150; Chatsworth, CA). The data from the left side of the bar were used for analysis. Analog signals from the force place and four linear position transducers were collected for every trial at 1,000 Hz using a BNC-2010 interface box with an analog-to-digital card (National Instruments PCI-6014; Austin, TX). 
LabVIEW (National Instruments, Version 8.6) was used for recording and analyzing the data. Signals from the force platform and linear position transducers were filtered using a fourth order, low pass Butterworth digital filter with a cut off frequency of $50 \mathrm{~Hz}$ and $10 \mathrm{~Hz}$ respectively. The force platform and linear position transducer voltage outputs were converted into vertical ground-reaction force and displacement, respectively, from laboratory calibrations. The rate of force development (RFD) was determined by calculating the rate of change of force from the trough of the eccentric muscle action to the peak of the concentric muscle action in the force time curve (24). The vertical velocity of the movement was determined using a first-order derivative of the displacement data. Power output was calculated as the product of the vertical velocity and vertical ground-reaction force data. This data collection and analysis procedure has been validated previously (7).

Paragraph 13. Additionally, the accelerometer data was imported and into LabVIEW (National Instruments, Version 8.6) and analyzed. Force was calculated by multiplying the acceleration at any given time point by the mass of the body. Velocity data were derived by single integration of the acceleration data with respect to time. Power output was then calculated as the product of force and velocity. Additionally, flight time was calculated by determining the time between the highest and lowest vertical velocity and then placed in an equation along with gravity to determine displacement (6).

Paragraph 14. Furthermore, the data was downsampled by removing an even number of samples along the curve (i.e. every other data point was removed to downsample from 500 to 250, every forth data point was removed to downsample from 500 to 125 and every tenth data point was removed to downsample from 500 to 50). This method allowed for the comparison of 
sampling rates between the force platform + linear position transducer system frequency of 1000 $\mathrm{Hz}$ and the accelerometer frequencies of $500 \mathrm{~Hz}, 250 \mathrm{~Hz}, 125$, and $50 \mathrm{~Hz}$.

Paragraph 15. Statistical Analyses. Statistical analyses were carried out using SPSS 17.0 for Windows (SPSS, Inc., Chicago, IL). An alpha level of 0.05 was used to indicate statistical significance with all analyses. All values are reported as means \pm standard deviations (SD). All analyses were performed on the total subject population as well as the population subdivided by gender.

Paragraph 16. To examine the reliability of the accelerometer and the force platform + linear position transducer system, several measurements were used: 1) coefficients of variance (CV) and 2) intra-class correlations (ICC) between the individual jump trials were calculated for the force platform + linear position transducer system and the accelerometer data. With both analyses, the $90 \%$ confidence intervals were calculated and reported. A minimum lower limit for the $90 \%$ confidence interval was set at ICC as being $>0.70$ in order to be considered reliable (3). As a general rule, the smaller the CV the more reliable the measure (17). However, for most biological research a $\mathrm{CV}<15 \%$ is considered as having acceptable reproducibility (1). Therefore, the upper limit for reliability for the tests performed in this investigation is a $\mathrm{CV}$ of $15 \%$.

Paragraph 17. A $1 \times 5$ repeated measures analysis of variance (ANOVA) was used to determine if significant differences existed between the force platform-linear position transducer system and the multiple sampling frequencies tested with the accelerometer system. When significant $\mathrm{F}$ values were determined, paired comparisons coupled with a Holm's Bonferroni adjustment to control for type I errors were used to determine the significant differences (15). 
Paragraph 18. In order to determine the validity of the accelerometer system, the methods of Hopkins et al. (18) in which linear regression analyses were used to determine a calibration equation, the standard error of the estimate (SEE), and a validity correlation coefficient. Simple linear regression analyses were performed because they provide superior statistics when performing validity studies (18). Specifically, the regression equation provides a trustworthy estimate of the bias of the test to the criterion measure, while the SEE and validity correlation help in the determination of the suitability of the measure for clinical assessments and a minimum limit of $r>0.80$ was set for a correlation to be considered high. Additionally, the linear regression analysis does not express the artifactual bias for measure with substantially different errors that can be found with Bland-Altman plots and does not represent random error in measurements provided by the instrument. (18). Because of these issues, Bland-Altman plots were not performed in the analysis of the data collected in this study.

\section{RESULTS}

Paragraph 19. Reliability. The accelerometer produced similar indices of reliability to those seen with the force platform + linear position transducer system (Table 3.1). Specifically, the accelerometer was found to be reliable (lower limit of the $90 \%$ confidence interval $=$ ICC > 0.70) for peak force, RFD, peak power, peak velocity and peak displacement for each sampling frequency tested when examining the total sample pool and male only group. The female group was similar for all variables excluding peak velocity, which did meet the lower limit of the $90 \%$ confidence interval for the ICC. Similarly, when looking at the total sample pool, the force platform + linear position transducer system was equally reliable based upon a minimum ICC cut-off (Table 3.1).

- Insert Table 3.1, 3.2, 3.3 - 
Paragraph 20. Further evaluation of reliability, with the use of the CV, determined acceptable reliability $(\mathrm{CV}<15.0 \%)$ with the accelerometer at all sampling rates when examining the peak force, peak velocity, peak power, flight time, and vertical displacement with the total subject pool (Table 3.1). The same variables met the $15 \% \mathrm{CV}$ limit when examining the results based upon gender (Table 3.2 and 3.3). The only measure that did not meet the CV reliability cut-off was the RFD, which consistently produced a CV > 15\% regardless of the accelerometer sampling frequency, gender tested or instrumentation used (accelerometer or force platform + linear position transducer) (Tables 3.2-3.3)

Paragraph 21. Validity. The linear regression analysis for the total subject pool revealed that all of the accelerometer sampling frequencies were significantly $(\mathrm{p}<0.001)$ correlated with force platform + linear position transducer system for the variables collected in this investigation (Table 3.4). In particular, the $250 \mathrm{~Hz}$ sampling frequency exhibited very high significant correlations to the force platform + linear position transducer system for peak force, RFD, peak power, flight time and vertical displacement when examining the total subject pool and both the men's and women's data individually, but these were substantially weaker than those seen in the total sample pool (Table 3.4 and 3.5). Additionally, with each variable tested, the $250 \mathrm{~Hz}$ sampling frequency commonly exhibited the lowest SEE values (Table 3.5).

- Insert Table 3.4 and 3.5 -

Paragraph 22. Significant differences ( $\mathrm{p}<0.001)$ in peak force, RFD, peak power, velocity, flight time and vertical displacement were observed when comparing the force platform + linear position transducer system in the total sample, men and women only groups across the various accelerometer sampling rates (Table 3.6). Follow-up paired comparisons indicated that regardless of the sampling frequency, the accelerometer significantly $(\mathrm{p}<0.001)$ overestimated 
peak force, RFD, flight time and vertical displacement when compared to the force platform + linear position transducer system. Additionally, follow-up tests revealed that regardless of sampling frequency or subject population, the peak velocity and peak power determined by the accelerometer were significantly $(\mathrm{p}<0.001)$ less than that determined by the force platform + linear position transducer system. A summary of the vertical jump parameters compared between the accelerometer and force platform + linear position transducer system are presented in Table 3.6.

- Insert Table 3.6 -

\section{DISCUSSION}

Paragraph 23. The principal finding in this original investigation was that a tri-axial accelerometer is valid and reliable against a force platform + linear position transducer system, with the most valid results surfacing when the accelerometer sampled at $250 \mathrm{~Hz}$. Additionally, when looking at the groups by gender, the accelerometer was less reliable when used to assess jump performance in the women as compared to the men.

Paragraph 24. Reliability. Being one of the first studies to investigate the use of accelerometer systems to quantify vertical jump performance, the findings of the current study suggest the accelerometer is a reliable device at frequencies as low as $50 \mathrm{~Hz}$ for peak force, peak power, peak velocity, flight time and peak displacement when looking at the total subject pool. Similar results were seen for the males and females excluding the peak velocity, which did not make the reliability cut-off for females. This data is consistent with Hori et al. (19) who found peak power, peak force, and peak velocity to be reliable down to $25 \mathrm{~Hz}$ with a force platform. While the RFD made the ICC cut off at every sampling frequency, it did not achieve the CV cutoff at any of the sampling frequencies tested. This may have been due to how RFD was 
calculated as the average RFD throughout the eccentric and concentric phases of the jump. It is unclear whether similar results would be observed if assessing peak RFD (i.e. the highest RFD during a 5-50 ms period). However, peak RFD is commonly assessed during a squat vertical jump instead of a countermovement vertical due to inconsistencies in where the peak RFD occurs (i.e. eccentric or concentric phase) (19).

Paragraph 25. Validity. Validity of a device is equally as important for it is essential to be able to compare the norms of athletes to athletes in similar situations. The linear regression analysis for the total subject pool revealed that all of the accelerometer sampling frequencies were significantly $(\mathrm{p}<0.001)$ correlated with force platform + linear position transducer system for the variables collected in this investigation. The correlations were not high at $500 \mathrm{~Hz}$; however; reducing the sampling frequency revealed that peak force, peak power and flight time had statistically significant high $(\mathrm{r}=0.80)$ correlations at $250 \mathrm{~Hz}, 125 \mathrm{~Hz}$ and $50 \mathrm{~Hz}$, which are sufficient criteria to consider the variables valid. This is an important finding because peak power output during an athletic activity is commonly the most important variable associated with success $(13,23,27,35)$. Evidence supporting this finding can be seen in a study performed by Ruben et al. (29) in which increases in force, power and velocity were seen in hurdle hops after a potentiation protocol with an accelerometer sampling at $200 \mathrm{~Hz}$. Furthermore, Hori et al. (19) found that a force platform sampling at $500 \mathrm{~Hz}$ had less than $2 \%$ difference and nearly perfect correlations until dropping below $200 \mathrm{~Hz}$. Similar to the total sample pool, the men and women's data exhibited the highest correlations between the accelerometer and force platform + linear position transducer system at $250 \mathrm{~Hz}$, but were substantially weaker than those seen in the total sample pool. 
Paragraph 26. When collecting acceleration data with the accelerometer, it is evident that the acceleration can vary over time. Even though the subject is changing acceleration rates throughout the entire jump, it is recorded only at the time points determined by the sampling frequency (i.e. every 0.004 seconds if the sampling frequency is $250 \mathrm{~Hz}$ ) with the assumption that change between successive samples is linear (19). Essentially, the accelerometer may not detect rapid changes in acceleration if the sampling frequency is too low and thus the time interval between too high, such as 125 or $50 \mathrm{~Hz}(19)$. Moreover, the $250 \mathrm{~Hz}, 125 \mathrm{~Hz}$ and $50 \mathrm{~Hz}$ sampling frequencies may be more valid than $500 \mathrm{~Hz}$ due to the data at $500 \mathrm{~Hz}$ having too much variability with the extra samples; thus, decreasing the sampling frequency from $500 \mathrm{~Hz}$ may "smooth" the data without losing important parts of the curve that are seen when the sampling frequency is decreased to $250 \mathrm{~Hz}, 125 \mathrm{~Hz}$ or $50 \mathrm{~Hz}$.

Paragraph 27. Peak force, RFD, peak power, velocity, flight time and displacement values differed significantly between the accelerometer and the force platform + linear position transducer system across all sampling frequencies assessed (Table 3.6). Additionally, peak force, velocity, flight time and displacement values also differed significantly between the accelerometer and the force platform + linear position transducer system across all sampling frequencies assessed (Table 3.6). The accelerometer systematically overestimated $(\mathrm{p}<0.001)$ peak force, RFD, flight time and displacement and underestimated $(\mathrm{p}<0.001)$ peak velocity and peak power when compared to the force platform + linear position transducer system. It is unclear precisely what is driving these differences. However, it is theorized that force is overestimated due to an indirect measurement of force by way of acceleration, body mass and gravity. Furthermore, velocity is most likely underestimated due to integrating the acceleration data to calculate the vertical velocity, which can lead to noise amplification and the subsequent 
risk of erroneous data (34). Consequently, peak power is underestimated due to the inconsistencies in force and velocity. Also, due to the lower sampling frequency of the accelerometer compared to the force platform + linear position transducer system, the accelerometer may not be able to detect the integral parts of the velocity-time curve that it uses to calculate the flight time and thus the vertical displacement.

Paragraph 28. In conclusion, the findings of the current study suggest the accelerometer to be a reliable device for measuring for peak force, RFD, peak power, peak velocity and peak displacement in a countermovement jump using sampling frequencies between 50 to $500 \mathrm{~Hz}$. Significant positive correlations were observed between the accelerometer and the force platform + linear position transducer system in all performance characteristics assessed. However, the accelerometer significantly overestimated peak force, RFD, flight time and displacement and significantly underestimated peak velocity and peak power. Thus, the accelerometer can be considered valid for these variables, but not precise.

Paragraph 29. Despite no significant differences in the group averages of the performance characteristics across the different accelerometer sampling frequencies, the highest correlations and SEE values were observed at $250 \mathrm{~Hz}, 125$ and $50 \mathrm{~Hz}$. Although this data is valid and reliable, it lacks the precision of a force platform + linear position transducer system. Therefore, sport scientists and practitioners should consider $250 \mathrm{~Hz}$ the ideal sampling frequency due to the increased number of samples while still achieving the lower limit of validity. However, sampling frequencies as low as $50 \mathrm{~Hz}$ may be considered if there are limitations imposed by the manufacturer or when testing large teams and the decreased file size is needed. Thus, it is imperative that the sampling frequency is kept consistent across testing sessions and comparisons to norms performed cautiously. Finally, when examining across genders, the males 
produce more valid but equally as reliable results as the females across all sampling frequencies. The novel finding in this investigation is that sport scientists now have the ability to take this small, portable device into any strength and conditioning facility and produce valid, reliable and immediate results in important performance characteristics that occur in the vertical jump.

ACKNOWLEDGEMENTS: The authors of this investigation would like to thank the subjects who assisted with this project and Myotest Inc., which donated the accelerometer systems that were used in the performance of this investigation. The results of this study do not constitute an endorsement of the accelerometer used in this project by either the authors or the journal. Additionally, the results of the present study do not constitute endorsement by ACSM. 


\section{REFERENCES}

1. Ashley CD, and Weiss LW. Vertical jump performance and selected physiological characteristics of women. J Strength Cond Res. 1994;8(1):5-11.

2. Barnes JL. Relationship of jumping and agility performance in female volleyball athletes. $J$ Strength Cond Res. 2007;21(4):1192-6.

3. Baumgartner TA, Chung H. Confidence limits for intraclass reliability coefficients. Meas Phys Educ Exerc Sci. 2001;5(3):179-88.

4. Canavan PK, Garret GE, Armstrong LE. Kinematic and kinetic relationships between an olympic-style lift and the vertical jump. J Strength Cond Res. 1996;10(2):127-30.

5. Carlock J, Smith A, Hartman M, Morris R, Ciroslan D, Pierce KC, Newton RU, Stone MH. The relationship between vertical jump power estimates and weightlifting ability: a field test approach. J Strength Cond Res. 2004;18(3):534-9.

6. Casartelli N. Validity and reliability of the Myotest measurement system assessing vertical jump performance [thesis]. Zurich (CH): ETH Zurich; 2009. 62 p.

7. Cormie P, McBride JM, McCaulley GO. Validation of power measurement techniques in dynamic lower body resistance exercises. J Appl Biomech. 2007;23(2):103-18.

8. Costill DL, Miller SJ, Myers WC, Kehoe FM, Hoffman WH. Relationship among selected tests of explosive leg strength and power. Res $Q$. 1968;39(3):785-7.

9. Delecluse C, Roelants M, Diels R, Koninckx E, Verschueren S. Effects of whole body vibration training on muscle strength and sprint performance in sprint-trained athletes. Int $J$ Sports Med. 2005;26(8):662-8. 
10. Dugan EL, Doyle TL, Humphries B, Hasson CJ, Newton RU. Determining the optimal load for jump squats: a review of methods and calculations. J Strength Cond Res. 2004;18(3):66874.

11. Erculj F. Impact of fatigue on the position of the release arm and shoulder girdle over a longer shooter distance for an elite basketball player. J Strength Cond Res. 2009;23(3):102936.

12. Garhammer J. Equipment for the development of athletic strength and power. Nat Strength Coaches Assoc J. 1981;3(6):24-6.

13. Garhammer J. A review of power output studies of Olympic and powerlifting: methodology, performance prediction, and evaluation tests. J. Strength Cond. Res. 1993;7(2):76-8.

14. Haff GG, Stone MH, O'Bryant HS, Harman E, Dinan CN, Johnson R, Han KH. Force-time dependent characteristics of dynamic and isometric muscle actions. J Strength Cond Res. 1997;11(4):269-72.

15. Holm S. A simple sequentially rejective multiple test procedure. Scand J Statistics. 1979;6:65-70.

16. Hopkins WG. Measures of reliability in sports medicine and science. Sports Med. 2000;30(1):1-15.

17. Hopkins WG. Reliability of Power in Physical Performance Tests. Sports Med. 2001;31(3):211-34.

18. Hopkins WG, Marshall SW, Batterham AM, Hanin J. Progressive statistics for studies in sports medicine and exercise science. Med Sci Sports Exerc. 2009;41(1):3-13.

19. Hori N, Newton RU, Kawamori N, McGuigan M, Kraemer W, Nosaka K. Reliability of performance measurements derived from ground reaction force data during 
countermovement jump and the influence of sampling frequency. $J$ Strength Cond Res. 2009;23(3):874-82.

20. Isaacs SD. Comparison of the vertec and just jump systems for measuring height of vertical jump by young children. Percept Mot Skills. 1998;86(2):659-63.

21. Jeffreys I. Total Soccer Fitness. Monterey (CA): Coaches Choice; 2007, 233 p.

22. Jeffreys I. Movement Training for Field Sports: Soccer. Strength and Cond J. 2008;30(4):1927.

23. Kauhanen H, Komi PV, Hakkinen K. Standardization and validation of the body weight adjustment regression equations in Olympic weightlifting. J Strength Cond Res. 2002;16(1):58-74.

24. Kawamori N, Rossi SJ, Justice BD, Haff EE, Pistilli EE, O'Bryant HS, Stone MH, Haff GG. Peak force and rate of force development during isometric and dynamic mid-thigh clean pulls performed at various intensities. J Strength Cond Res. 2006;20(3):483-91.

25. Major JA, Sands WA, McNeal JR, Paine DD, Kipp R. Design, construction, and validation of a portable one-dimensional force platform. J Strength Cond Res. 1998;12(1):37-41.

26. Markovic G, Dizdar D, Juckic I, Cardinale M. Reliability and factorial validity of squat and countermovement jump tests. J Strength Cond Res. 2004;18(3):551-5.

27. McBride JM, Triplett-McBride T, Davie A, Newton RU. A comparison of strength and power characteristics between power lifters, olympic lifters, and sprinters. J Strength Cond Res. 1999;13(1):58-66.

28. Mero A, Luhtanen P, Viitasalo JT, Komi PV. Relationships between maximal running velocity, muscle fiber characteristics, force production and force relaxation of sprinters. Scand J Sports Sci. 1981;3(1):16-22. 
29. Ruben RM, Bibbee CA, Childress MA, Harman MS, Reed KP, Haff GG. The acute effects of heavy-load squats on performance during plyometric jumps. J Strength Cond Res. 2010;24(2):358-69.

30. Sato K, Smith SL, Sands WA. Validation of an accelerometer for measuring sport performance. J Strength Cond Res. 2009;23(1):341-7.

31. Tessitore A, Meeusen R, Pagano R, Benvenuti C, Tiberi M, Capranica L. Effectiveness of active versus passive recovery strategies after futsal games. J Strength Cond Res. 2008;22(5):1402-12.

32. Whaley O, McClure R. Another perspective on teaching the pulling movements. Strength and Cond J. 1997;19(2):58-61.

33. Wisløff U, Castagna C, Helgerud J, Jones R, Hoff J. Strong correlation of maximal squat strength with sprint performance and vertical jump height in elite soccer players. Br J Sports Med. 2004;38(3):285-8.

34. Wood GA. Data smoothing and differentiation procedures in biomechanics. Exerc Sport Sci Rev. 1982;10(10):308-62.

35. Young W, MacDonald C, Heggen T, Fitzpatrick J. An evaluation of the specificity, validity and reliability of jumping tests. J Sports Med Phys Fitness. 1997;37(4):240-5. 
Table 3.1: Reliability Data for the Accelerometer and Force Platform + Linear Position Transducer System for the Total Sample Population.

\begin{tabular}{|c|c|c|c|c|c|c|c|c|}
\hline \multirow{2}{*}{$\begin{array}{l}\text { Variable } \\
\text { Peak Force }\end{array}$} & \multirow{2}{*}{$\begin{array}{c}\text { System } \\
\text { Accelerometer }\end{array}$} & \multirow{2}{*}{$\begin{array}{c}\begin{array}{c}\text { Sampling } \\
\text { Rate } \\
(\mathrm{Hz})\end{array} \\
500\end{array}$} & \multirow{2}{*}{$\begin{array}{l}\text { ICC } \\
0.95\end{array}$} & \multicolumn{2}{|c|}{$90 \% \mathrm{CI}$} & \multirow{2}{*}{$\begin{array}{r}\mathrm{CV} \\
(\%) \\
6.9\end{array}$} & \multicolumn{2}{|c|}{$90 \% \mathrm{CI}$} \\
\hline & & & & 0.93 & - 0.96 & & 6.5 & 7.4 \\
\hline & & 250 & 0.95 & 0.93 & - 0.96 & 6.7 & 6.3 & 7.2 \\
\hline & & 125 & 0.95 & 0.93 & - 0.96 & 6.8 & 6.4 & 7.3 \\
\hline & & 50 & 0.95 & 0.93 & - 0.96 & 6.9 & 6.5 & 7.4 \\
\hline & $\begin{array}{l}\mathrm{FP}+\mathrm{LPT} \\
\text { System }\end{array}$ & 1000 & 0.97 & 0.96 & - 0.98 & 4.0 & 3.7 & 4.3 \\
\hline \multirow{5}{*}{$\begin{array}{l}\text { Rate of Force } \\
\text { Development }\end{array}$} & Accelerometer & 500 & 0.85 & 0.81 & - $\quad 0.89$ & 21.1 & 19.7 & -22.8 \\
\hline & & 250 & 0.86 & 0.82 & - 0.89 & 20.9 & 19.5 & - 22.5 \\
\hline & & 125 & 0.86 & 0.82 & - 0.89 & 20.5 & 19.2 & - 22.1 \\
\hline & & 50 & 0.86 & 0.82 & - 0.90 & 19.8 & 18.5 & - 21.3 \\
\hline & $\begin{array}{l}\mathrm{FP}+\mathrm{LPT} \\
\text { System }\end{array}$ & 1000 & 0.83 & 0.78 & - 0.87 & 21.9 & 20.5 & -23.6 \\
\hline \multirow[t]{5}{*}{ Peak Power } & Accelerometer & 500 & 0.90 & 0.87 & - 0.93 & 13.4 & 12.5 & - 14.3 \\
\hline & & 250 & 0.90 & 0.87 & - 0.93 & 13.3 & 12.4 & - $\quad 14.2$ \\
\hline & & 125 & 0.90 & 0.87 & - 0.93 & 13.2 & 12.3 & - 14.1 \\
\hline & & 50 & 0.90 & 0.88 & - 0.93 & 13.1 & 12.3 & -14.1 \\
\hline & $\begin{array}{l}\text { FP + LPT } \\
\text { System }\end{array}$ & 1000 & 0.95 & 0.94 & - 0.96 & 7.8 & 7.3 & 8.5 \\
\hline \multirow[t]{5}{*}{ Peak Velocity } & Accelerometer & 500 & 0.81 & 0.75 & -0.85 & 7.9 & 7.4 & 8.4 \\
\hline & & 250 & 0.80 & 0.75 & - 0.85 & 7.9 & 7.4 & 8.5 \\
\hline & & 125 & 0.81 & 0.75 & - 0.85 & 7.9 & 7.4 & 8.5 \\
\hline & & 50 & 0.80 & 0.75 & - 0.85 & 7.9 & 7.4 & 8.5 \\
\hline & $\begin{array}{l}\text { FP + LPT } \\
\text { System }\end{array}$ & 1000 & 0.83 & 0.78 & - 0.87 & 6.7 & 6.3 & 7.2 \\
\hline \multirow[t]{5}{*}{ Flight Time } & Accelerometer & 500 & 0.94 & 0.92 & - $\quad 0.95$ & 2.7 & 2.5 & 2.9 \\
\hline & & 250 & 0.94 & 0.92 & - 0.95 & 2.8 & 2.6 & 3.0 \\
\hline & & 125 & 0.94 & 0.92 & - 0.96 & 2.6 & 2.5 & 2.8 \\
\hline & & 50 & 0.93 & 0.91 & - 0.95 & 2.8 & 2.7 & 3.0 \\
\hline & $\begin{array}{l}\mathrm{FP}+\mathrm{LPT} \\
\text { System }\end{array}$ & 1000 & 0.96 & 0.95 & - 0.97 & 5.4 & 5.1 & 5.8 \\
\hline \multirow[t]{5}{*}{ Vertical Displacement } & Accelerometer & 500 & 0.94 & 0.92 & -0.95 & 5.5 & 5.2 & 5.9 \\
\hline & & 250 & 0.94 & 0.92 & - 0.95 & 5.6 & 5.3 & 6.0 \\
\hline & & 125 & 0.94 & 0.92 & - 0.95 & 5.4 & 5.0 & - $\quad 5.7$ \\
\hline & & 50 & 0.97 & 0.96 & - 0.98 & 5.7 & 5.4 & 6.2 \\
\hline & $\begin{array}{l}\text { FP+ LPT } \\
\text { System }\end{array}$ & 1000 & 0.88 & 0.85 & -0.91 & 8.3 & 7.7 & 8.8 \\
\hline
\end{tabular}

Note: $\mathrm{LPT}=$ linear position transducer, $\mathrm{FP}=$ force platform 
Table 3.2: Reliability Data for the Accelerometer and Force Platform + Linear Position Transducer System for the Men.

\begin{tabular}{|c|c|c|c|c|c|c|c|c|c|}
\hline \multirow{2}{*}{$\begin{array}{l}\text { Variable } \\
\text { Peak Force }\end{array}$} & \multirow{2}{*}{$\begin{array}{c}\text { System } \\
\text { Accelerometer }\end{array}$} & \multirow{2}{*}{$\begin{array}{c}\begin{array}{c}\text { Sampling } \\
\text { Rate } \\
(\mathrm{Hz})\end{array} \\
500\end{array}$} & \multirow{2}{*}{$\begin{array}{c}\text { ICC } \\
0.95\end{array}$} & \multicolumn{2}{|c|}{$90 \% \mathrm{CI}$} & \multirow{2}{*}{$\begin{array}{r}\mathrm{CV} \\
(\%) \\
6.5\end{array}$} & \multicolumn{3}{|c|}{$90 \% \mathrm{CI}$} \\
\hline & & & & 0.93 & -0.96 & & 6.1 & & 7.1 \\
\hline & & 250 & 0.95 & 0.93 & - 0.96 & 6.2 & 5.8 & - & 6.7 \\
\hline & & 125 & 0.95 & 0.93 & -0.96 & 6.4 & 5.9 & - & 6.9 \\
\hline & & 50 & 0.95 & 0.93 & -0.96 & 6.4 & 5.9 & - & 6.9 \\
\hline & $\begin{array}{l}\mathrm{FP}+\mathrm{LPT} \\
\text { System }\end{array}$ & 1000 & 0.96 & 0.95 & -0.97 & 4.1 & 3.8 & - & 4.4 \\
\hline \multirow{5}{*}{$\begin{array}{l}\text { Rate of Force } \\
\text { Development }\end{array}$} & Accelerometer & 500 & 0.90 & 0.88 & -0.93 & 19.4 & 18.0 & - & 21.1 \\
\hline & & 250 & 0.91 & 0.88 & - 0.93 & 19.1 & 17.7 & - & 20.8 \\
\hline & & 125 & 0.90 & 0.87 & - 0.93 & 19.4 & 18.0 & . & 21.2 \\
\hline & & 50 & 0.91 & 0.88 & - 0.93 & 18.4 & 17.1 & - & 20.1 \\
\hline & $\begin{array}{l}\text { FP + LPT } \\
\text { System }\end{array}$ & 1000 & 0.89 & 0.86 & -0.91 & 19.2 & 17.8 & 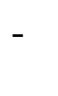 & 20.9 \\
\hline \multirow[t]{5}{*}{ Peak Power } & Accelerometer & 500 & 0.87 & 0.84 & -0.90 & 12.5 & 11.6 & & 13.6 \\
\hline & & 250 & 0.88 & 0.84 & - 0.91 & 12.2 & 11.4 & & 13.3 \\
\hline & & 125 & 0.88 & 0.85 & - 0.91 & 12.1 & 11.2 & & 13.1 \\
\hline & & 50 & 0.88 & 0.85 & - 0.91 & 12.1 & 11.2 & & 13.1 \\
\hline & $\begin{array}{l}\text { FP + LPT } \\
\text { System }\end{array}$ & 1000 & 0.95 & 0.94 & - 0.96 & 6.8 & 6.3 & & 7.4 \\
\hline \multirow[t]{5}{*}{ Peak Velocity } & Accelerometer & 500 & 0.84 & 0.79 & - $\quad 0.87$ & 7.3 & 6.8 & - & 7.9 \\
\hline & & 250 & 0.83 & 0.79 & - $\quad 0.87$ & 7.4 & 6.8 & - & 8.0 \\
\hline & & 125 & 0.84 & 0.79 & - 0.87 & 7.3 & 6.8 & - & 7.9 \\
\hline & & 50 & 0.84 & 0.80 & - 0.88 & 7.2 & 6.7 & - & 7.9 \\
\hline & $\begin{array}{l}\text { FP + LPT } \\
\text { System }\end{array}$ & 1000 & 0.78 & 0.73 & - $\quad 0.83$ & 6.3 & 5.8 & - & 6.8 \\
\hline \multirow[t]{5}{*}{ Flight Time } & Accelerometer & 500 & 0.90 & 0.87 & -0.92 & 2.4 & 2.3 & - & 2.6 \\
\hline & & 250 & 0.87 & 0.84 & - 0.90 & 2.7 & 2.5 & - & 2.9 \\
\hline & & 125 & 0.90 & 0.88 & - 0.93 & 2.4 & 2.2 & - & 2.6 \\
\hline & & 50 & 0.90 & 0.87 & - 0.92 & 2.3 & 2.2 & - & 2.5 \\
\hline & $\begin{array}{l}\text { FP + LPT } \\
\text { System }\end{array}$ & 1000 & 0.92 & 0.90 & - 0.94 & 2.5 & 2.3 & - & 2.7 \\
\hline \multirow[t]{5}{*}{ Vertical Displacement } & Accelerometer & 500 & 0.90 & 0.87 & - 0.92 & 4.9 & 4.6 & - & 5.3 \\
\hline & & 250 & 0.87 & 0.84 & - 0.90 & 5.4 & 5.0 & - & 5.8 \\
\hline & & 125 & 0.91 & 0.88 & - 0.93 & 4.6 & 4.3 & - & 5.0 \\
\hline & & 50 & 0.90 & 0.87 & - 0.92 & 4.8 & 4.5 & - & 5.2 \\
\hline & $\begin{array}{l}\text { FP + LPT } \\
\text { System }\end{array}$ & 1000 & 0.83 & 0.79 & -0.87 & 6.5 & 6.0 & - & 7.0 \\
\hline
\end{tabular}

Note: $\mathrm{LPT}=$ linear position transducer, $\mathrm{FP}=$ force platform 
Table 3.3: Reliability Data for the Accelerometer and Force Platform + Linear Position Transducer System for the Women

\begin{tabular}{|c|c|c|c|c|c|c|c|c|}
\hline \multirow{2}{*}{$\begin{array}{l}\text { Variable } \\
\text { Peak Force }\end{array}$} & \multirow{2}{*}{$\begin{array}{c}\text { System } \\
\text { Accelerometer }\end{array}$} & \multirow{2}{*}{$\begin{array}{c}\text { Sampling } \\
\begin{array}{c}\text { Rate } \\
(\mathrm{Hz})\end{array} \\
500\end{array}$} & \multirow{2}{*}{$\begin{array}{l}\text { ICC } \\
0.89\end{array}$} & \multicolumn{2}{|c|}{$90 \% \mathrm{CI}$} & \multirow{2}{*}{$\begin{array}{c}\begin{array}{l}\text { CV } \\
(\%)\end{array} \\
7.2\end{array}$} & \multicolumn{2}{|c|}{$90 \% \mathrm{CI}$} \\
\hline & & & & 0.86 & - 0.92 & & 6.7 & 7.8 \\
\hline & & 250 & 0.89 & 0.86 & - 0.92 & 7.2 & 6.7 & 7.8 \\
\hline & & 125 & 0.89 & 0.86 & - 0.92 & 7.2 & 6.7 & 7.8 \\
\hline & & 50 & 0.89 & 0.85 & - 0.91 & 7.3 & 6.8 & 8.0 \\
\hline & $\begin{array}{l}\text { FP + LPT } \\
\text { System }\end{array}$ & 1000 & 0.96 & 0.95 & - 0.97 & 3.3 & 3.1 & 3.6 \\
\hline \multirow{5}{*}{$\begin{array}{l}\text { Rate of Force } \\
\text { Development }\end{array}$} & Accelerometer & 500 & 0.75 & 0.69 & -0.80 & 22.8 & 21.1 & -24.9 \\
\hline & & 250 & 0.75 & 0.69 & - 0.80 & 22.7 & 21.0 & -24.8 \\
\hline & & 125 & 0.76 & 0.70 & - 0.81 & 21.7 & 20.1 & -23.6 \\
\hline & & 50 & 0.76 & 0.71 & - 0.81 & 21.2 & 19.6 & - 23.1 \\
\hline & $\begin{array}{l}\text { FP + LPT } \\
\text { System }\end{array}$ & 1000 & 0.73 & 0.66 & - 0.78 & 24.4 & 22.5 & -26.6 \\
\hline \multirow[t]{5}{*}{ Peak Power } & Accelerometer & 500 & 0.79 & 0.74 & - 0.84 & 14.0 & 13.0 & -15.3 \\
\hline & & 250 & 0.79 & 0.74 & - 0.84 & 14.0 & 13.0 & -15.3 \\
\hline & & 125 & 0.79 & 0.74 & - 0.84 & 14.1 & 13.0 & -15.3 \\
\hline & & 50 & 0.79 & 0.74 & - 0.84 & 13.9 & 12.9 & -15.1 \\
\hline & $\begin{array}{l}\text { FP + LPT } \\
\text { System }\end{array}$ & 1000 & 0.88 & 0.85 & - 0.91 & 8.8 & 8.1 & 9.5 \\
\hline \multirow[t]{5}{*}{ Peak Velocity } & Accelerometer & 500 & 0.64 & 0.56 & - 0.71 & 8.5 & 7.9 & 9.2 \\
\hline & & 250 & 0.64 & 0.56 & - 0.71 & 8.5 & 7.9 & 9.2 \\
\hline & & 125 & 0.64 & 0.56 & - 0.71 & 8.5 & 7.9 & 9.2 \\
\hline & & 50 & 0.63 & 0.56 & - 0.71 & 8.5 & 7.9 & 9.2 \\
\hline & $\begin{array}{l}\text { FP + LPT } \\
\text { System }\end{array}$ & 1000 & 0.71 & 0.65 & - 0.77 & 7.1 & 6.3 & 7.2 \\
\hline \multirow[t]{5}{*}{ Flight Time } & Accelerometer & 500 & 0.88 & 0.85 & - 0.91 & 3.0 & 2.8 & 3.3 \\
\hline & & 250 & 0.88 & 0.85 & - 0.91 & 3.0 & 2.8 & 3.2 \\
\hline & & 125 & 0.87 & 0.84 & - 0.90 & 3.1 & 2.9 & 3.3 \\
\hline & & 50 & 0.85 & 0.81 & - 0.88 & 3.3 & 3.0 & 3.5 \\
\hline & $\begin{array}{l}\text { FP + LPT } \\
\text { System }\end{array}$ & 1000 & 0.92 & 0.90 & - 0.94 & 2.9 & 2.7 & 3.1 \\
\hline \multirow[t]{5}{*}{ Vertical Displacement } & Accelerometer & 500 & 0.88 & 0.85 & - 0.91 & 6.1 & 5.7 & 6.6 \\
\hline & & 250 & 0.88 & 0.85 & - 0.91 & 5.9 & 5.5 & 6.4 \\
\hline & & 125 & 0.88 & 0.85 & - 0.91 & 6.1 & 5.6 & 6.6 \\
\hline & & 50 & 0.97 & 0.96 & - 0.98 & 6.5 & 6.1 & 7.1 \\
\hline & $\begin{array}{l}\text { FP + LPT } \\
\text { System }\end{array}$ & 1000 & 0.81 & 0.76 & -0.85 & 9.7 & 9.0 & -10.5 \\
\hline
\end{tabular}

Note: $\mathrm{LPT}=$ linear position transducer, $\mathrm{FP}=$ force platform 
Table 3.4: Linear Regression Equations between Force Platform + Linear Position Transducer and Accelerometer Data.

\begin{tabular}{lcccccl}
\hline FP + LPT & Accelerometer & $\mathrm{r}$ & $\mathrm{p}$ & $\mathrm{R}^{2}$ & $\mathrm{SEE}$ & Equation \\
\hline \multirow{4}{*}{ Peak Force } & $500 \mathrm{~Hz}$ & 0.72 & $<0.001$ & 0.52 & 307.9 & $\mathrm{y}=0.439 \mathrm{x}+636.7$ \\
& $250 \mathrm{~Hz}$ & 0.92 & $<0.001$ & 0.85 & 171.9 & $\mathrm{y}=0.561 \mathrm{x}+342.1$ \\
& $125 \mathrm{~Hz}$ & 0.80 & $<0.001$ & 0.64 & 268.6 & $\mathrm{y}=0.485 \mathrm{x}+526.4$ \\
& $50 \mathrm{~Hz}$ & 0.92 & $<0.001$ & 0.85 & 176.4 & $\mathrm{y}=0.553 \mathrm{x}+364.9$ \\
\hline & $500 \mathrm{~Hz}$ & 0.53 & $<0.001$ & 0.28 & 1899.1 & $\mathrm{y}=0.273 \mathrm{x}+2156.0$ \\
Rate of Force & $250 \mathrm{~Hz}$ & 0.70 & $<0.001$ & 0.49 & 1603.9 & $\mathrm{y}=0.358 \mathrm{x}+1456.0$ \\
Development & $125 \mathrm{~Hz}$ & 0.52 & $<0.001$ & 0.27 & 1922.6 & $\mathrm{y}=0.273 \mathrm{x}+221.0$ \\
& $50 \mathrm{~Hz}$ & 0.70 & $<0.001$ & 0.49 & 1592.0 & $\mathrm{y}=0.400 \mathrm{x}+1319.0$ \\
\hline & $500 \mathrm{~Hz}$ & 0.68 & $<0.001$ & 0.46 & 1427.2 & $\mathrm{y}=0.766 \mathrm{x}+1678.0$ \\
Peak Power & $250 \mathrm{~Hz}$ & 0.86 & $<0.001$ & 0.73 & 1011.3 & $\mathrm{y}=0.963 \mathrm{x}+852.7$ \\
& $125 \mathrm{~Hz}$ & 0.83 & $<0.001$ & 0.68 & 1095.3 & $\mathrm{y}=0.933 \mathrm{x}+984.6$ \\
& $50 \mathrm{~Hz}$ & 0.86 & $<0.001$ & 0.73 & 1009.0 & $\mathrm{y}=0.970 \mathrm{x}+848.0$ \\
\hline \multirow{4}{*}{ Peak Velocity } & $500 \mathrm{~Hz}$ & 0.38 & $<0.001$ & 0.14 & 0.456 & $\mathrm{y}=0.384 \mathrm{x}+2.081$ \\
& $250 \mathrm{~Hz}$ & 0.46 & $<0.001$ & 0.21 & 0.437 & $\mathrm{y}=0.467 \mathrm{x}+1.836$ \\
& $125 \mathrm{~Hz}$ & 0.46 & $<0.001$ & 0.21 & 0.439 & $\mathrm{y}=0.461 \mathrm{x}+1.852$ \\
& $50 \mathrm{~Hz}$ & 0.47 & $<0.001$ & 0.22 & 0.437 & $\mathrm{y}=0.470 \mathrm{x}+1.828$ \\
\hline \multirow{4}{*}{ Flight Time } & $500 \mathrm{~Hz}$ & 0.63 & $<0.001$ & 0.40 & 0.051 & $\mathrm{y}=0.644 \mathrm{x}+0.098$ \\
& $250 \mathrm{~Hz}$ & 0.83 & $<0.001$ & 0.69 & 0.037 & $\mathrm{y}=0.848 \mathrm{x}-0.026$ \\
& $125 \mathrm{~Hz}$ & 0.84 & $<0.001$ & 0.71 & 0.036 & $\mathrm{y}=0.853 \mathrm{x}-0.032$ \\
& $50 \mathrm{~Hz}$ & 0.83 & $<0.001$ & 0.68 & 0.037 & $\mathrm{y}=0.852 \mathrm{x}-0.042$ \\
\hline & $500 \mathrm{~Hz}$ & 0.60 & $<0.001$ & 0.36 & 0.070 & $\mathrm{y}=0.549 \mathrm{x}+0.155$ \\
Vertical & $250 \mathrm{~Hz}$ & 0.75 & $<0.001$ & 0.56 & 0.058 & $\mathrm{y}=0.678 \mathrm{x}+0.094$ \\
Displacement & $125 \mathrm{~Hz}$ & 0.76 & $<0.001$ & 0.58 & 0.057 & $\mathrm{y}=0.675 \mathrm{x}+0.092$ \\
& $50 \mathrm{~Hz}$ & 0.75 & $<0.001$ & 0.56 & 0.058 & $\mathrm{y}=0.663 \mathrm{x}+0.085$ \\
\hline
\end{tabular}

Note: $\mathrm{SEE}=$ Standard Error of the Estimate, $\mathrm{LPT}=$ linear position transducer, FP $=$ force platform 
Table 3.5: Linear Regression Equations between Force Platform + Linear Position Transducer and Accelerometer Data for Men and Women.

\begin{tabular}{|c|c|c|c|c|c|c|c|c|c|c|c|}
\hline \multirow{2}{*}{$\mathrm{FP}+\mathrm{LPT}$} & \multirow{2}{*}{ Accelerometer } & \multicolumn{5}{|c|}{ Men } & \multicolumn{5}{|c|}{ Women } \\
\hline & & $\mathrm{r}$ & $\mathrm{p}$ & $\mathrm{R}^{2}$ & SEE & Equation & $\mathrm{r}$ & $\mathrm{p}$ & $\mathrm{R}^{2}$ & SEE & Equation \\
\hline \multirow{4}{*}{ Peak Force } & $500 \mathrm{~Hz}$ & 0.65 & $<0.001$ & 0.42 & 329.8 & $y=0.374 x+925.8$ & 0.75 & $<0.001$ & 0.56 & 221.1 & $y=0.393 x+615.0$ \\
\hline & $250 \mathrm{~Hz}$ & 0.88 & $<0.001$ & 0.77 & 204.1 & $y=0.500 x+558.3$ & 0.97 & $<0.001$ & 0.91 & 100.9 & $y=0.579 x+256.7$ \\
\hline & $125 \mathrm{~Hz}$ & 0.64 & $<0.001$ & 0.41 & 333.6 & $y=0.360 x+936.0$ & 0.95 & $<0.001$ & 0.90 & 103.2 & $y=0.578 x+257.7$ \\
\hline & $50 \mathrm{~Hz}$ & 0.88 & $<0.001$ & 0.77 & 209.3 & $y=0.486 x+596.7$ & 0.95 & $<0.001$ & 0.90 & 101.1 & $y=0.583 x+250.1$ \\
\hline \multirow{4}{*}{$\begin{array}{l}\text { Rate of Force } \\
\text { Development }\end{array}$} & $500 \mathrm{~Hz}$ & 0.60 & $<0.001$ & 0.36 & 2094.5 & $y=0.305 x+2331.0$ & 0.40 & $<0.001$ & 0.16 & 1524.2 & $y=0.188 x+2393.0$ \\
\hline & $250 \mathrm{~Hz}$ & 0.78 & $<0.001$ & 0.61 & 1630.5 & $y=0.391 x+1471.0$ & 0.47 & $<0.001$ & 0.23 & 1462.2 & $y=0.241 x+2057.0$ \\
\hline & $125 \mathrm{~Hz}$ & 0.51 & $<0.001$ & 0.26 & 2253.4 & $y=0.259 x+2691.0$ & 0.50 & $<0.001$ & 0.25 & 1439.8 & $y=0.268 x+1888.0$ \\
\hline & $50 \mathrm{~Hz}$ & 0.78 & $<0.001$ & 0.60 & 1650.7 & $y=0.426 x+1387.0$ & 0.49 & $<0.001$ & 0.24 & 1443.6 & $y=0.288 x+1844.0$ \\
\hline \multirow{4}{*}{ Peak Power } & $500 \mathrm{~Hz}$ & 0.66 & $<0.001$ & 0.44 & 1462.0 & $y=0.762 x+2253.0$ & 0.55 & 0.002 & 0.30 & 1055.6 & $y=0.457 x+2227.0$ \\
\hline & $250 \mathrm{~Hz}$ & 0.80 & $<0.001$ & 0.64 & 1182.9 & $y=0.879 x+1514.0$ & 0.83 & $<0.001$ & 0.69 & 709.9 & $y=0.865 x+944.1$ \\
\hline & $125 \mathrm{~Hz}$ & 0.74 & $<0.001$ & 0.55 & 1309.9 & $y=0.819 x+1821.0$ & 0.83 & $<0.001$ & 0.69 & 710.0 & $y=0.866 x+943.2$ \\
\hline & $50 \mathrm{~Hz}$ & 0.80 & $<0.001$ & 0.64 & 1182.7 & $y=0.885 x+1514.0$ & 0.83 & $<0.001$ & 0.69 & 704.6 & $y=0.875 x+929.8$ \\
\hline \multirow{4}{*}{$\begin{array}{l}\text { Peak } \\
\text { Velocity }\end{array}$} & $500 \mathrm{~Hz}$ & 0.27 & $<0.001$ & 0.07 & 0.426 & $y=0.240 x+2.756$ & 0.28 & $<0.001$ & 0.08 & 0.344 & $y=0.221 x+2.305$ \\
\hline & $250 \mathrm{~Hz}$ & 0.31 & $<0.001$ & 0.10 & 0.420 & $y=0.273 x+2.643$ & 0.35 & $<0.001$ & 0.12 & 0.335 & $y=0.308 x+2.073$ \\
\hline & $125 \mathrm{~Hz}$ & 0.30 & $<0.001$ & 0.09 & 0.422 & $y=0.264 x+2.672$ & 0.35 & $<0.001$ & 0.12 & 0.335 & $y=0.308 x+2.073$ \\
\hline & $50 \mathrm{~Hz}$ & 0.31 & $<0.001$ & 0.10 & 0.420 & $y=0.273 x+2.644$ & 0.35 & $<0.001$ & 0.12 & 0.335 & $Y=0.308 x+2.073$ \\
\hline \multirow{4}{*}{ Flight Time } & $500 \mathrm{~Hz}$ & 0.50 & $<0.001$ & 0.25 & 0.010 & $y=0.427 x+0.264$ & 0.50 & $<0.001$ & 0.25 & 0.039 & $y=0.366 x+0.228$ \\
\hline & $250 \mathrm{~Hz}$ & 0.73 & $<0.001$ & 0.53 & 0.032 & $y=0.809 x+0.143$ & 0.75 & $<0.001$ & 0.56 & 0.029 & $y=0.685 x+0.080$ \\
\hline & $125 \mathrm{~Hz}$ & 0.75 & $<0.001$ & 0.56 & 0.031 & $y=0.621 x+0.133$ & 0.76 & $<0.001$ & 0.57 & 0.029 & $y=0.670 x+0.055$ \\
\hline & $50 \mathrm{~Hz}$ & 0.74 & $<0.001$ & 0.54 & 0.032 & $y=0.624 x+0.124$ & 0.73 & $<0.001$ & 0.54 & 0.030 & $y=0.645 x+0.061$ \\
\hline \multirow{4}{*}{$\begin{array}{l}\text { Vertical } \\
\text { Displacement }\end{array}$} & $500 \mathrm{~Hz}$ & 0.52 & $<0.001$ & 0.27 & 0.060 & $y=0.630 x+0.059$ & 0.43 & $<0.001$ & 0.18 & 0.061 & $y=0.326 x+0.215$ \\
\hline & $250 \mathrm{~Hz}$ & 0.61 & $<0.001$ & 0.37 & 0.055 & $y=0.478 x+0.213$ & 0.64 & $<0.001$ & 0.41 & 0.052 & $y=0.618 x+0.101$ \\
\hline & $125 \mathrm{~Hz}$ & 0.61 & $<0.001$ & 0.38 & 0.055 & $y=0.476 x+0.212$ & 0.65 & $<0.001$ & 0.42 & 0.051 & $y=0.621 x+0.097$ \\
\hline & $50 \mathrm{~Hz}$ & 0.61 & $<0.001$ & 0.37 & 0.056 & $y=0.472 x+0.205$ & 0.62 & $<0.001$ & 0.38 & 0.053 & $y=0.580 x+0.103$ \\
\hline
\end{tabular}

Note: $\mathrm{SEE}=$ Standard Error of the Estimate, FP $=$ Force platform, LPT $=$ Linear position transducer 
Table 3.6: Vertical Jump Data for Men and Women

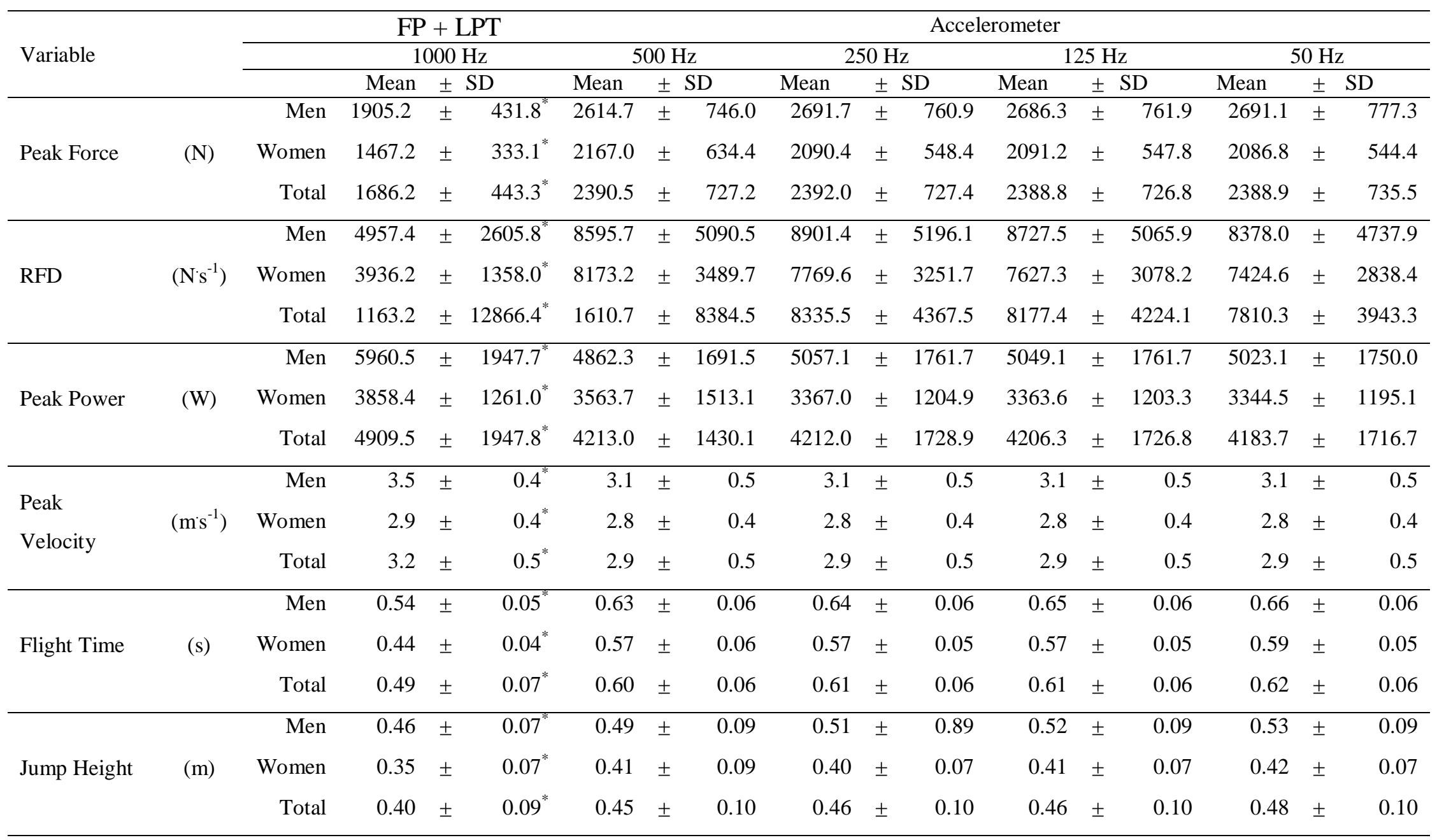

Note: RFD = Rate of force development.

* = significantly different than accelerometer at $500 \mathrm{~Hz}, 250 \mathrm{~Hz}, 125 \mathrm{~Hz}$, and $50 \mathrm{~Hz}$ p $\leq 0.001$. 


\section{Chapter 4. Appendix}

Figure 4.1 Project Timeline and Design

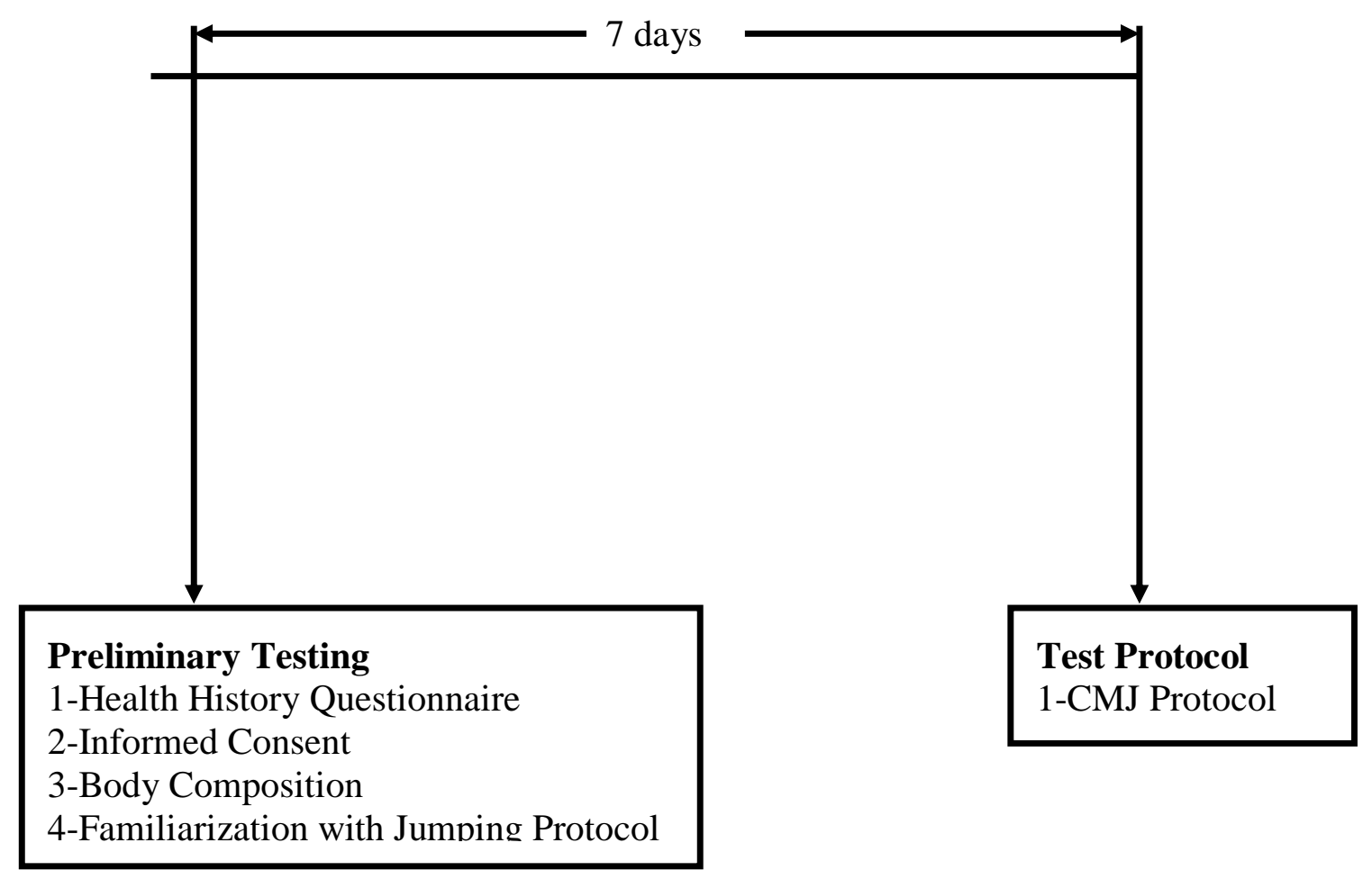


Figure 4.2: Force Platform + 2-Linear Position Transducer System with Accelerometer

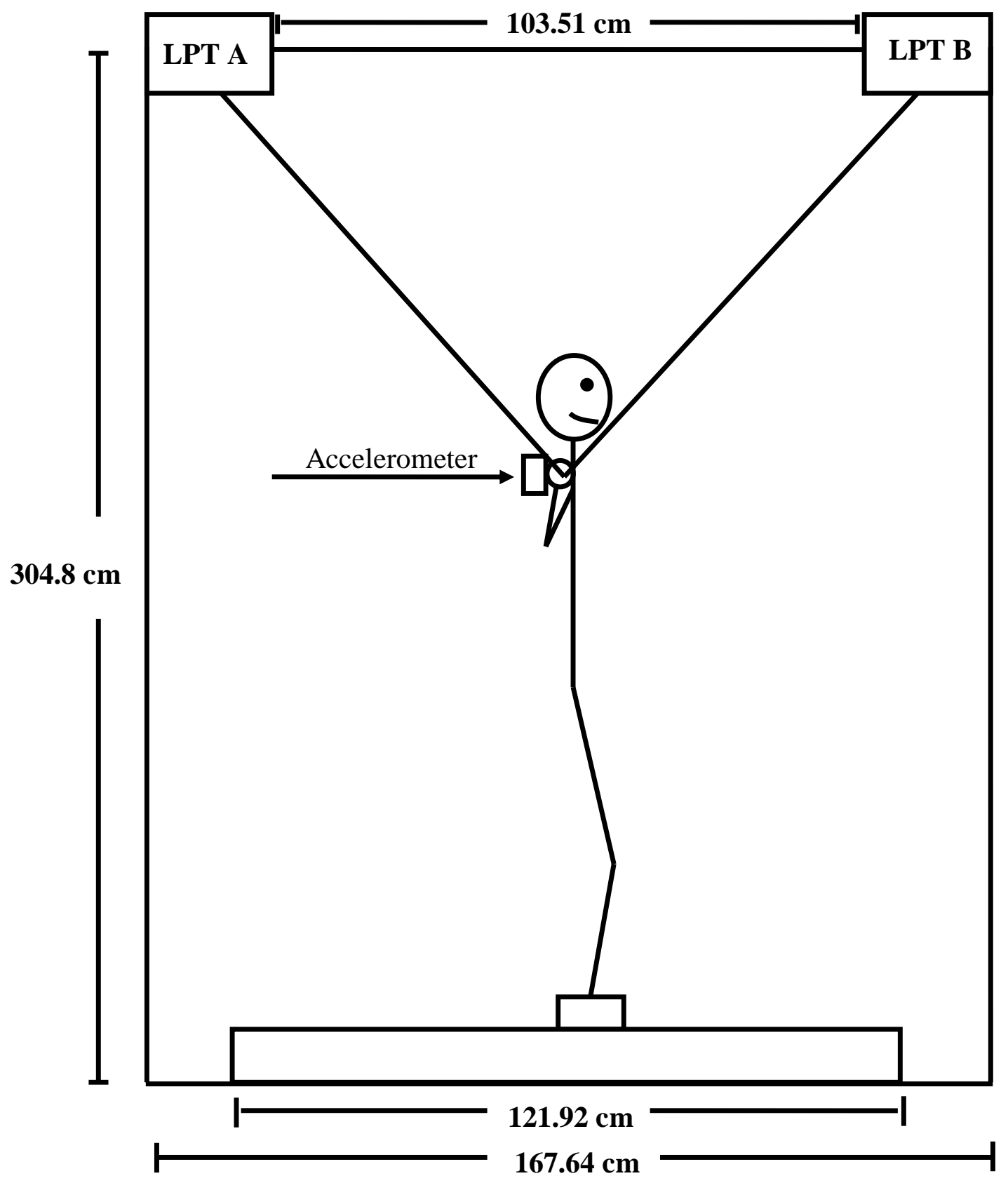


Figure 4.3: Experimental Protocol

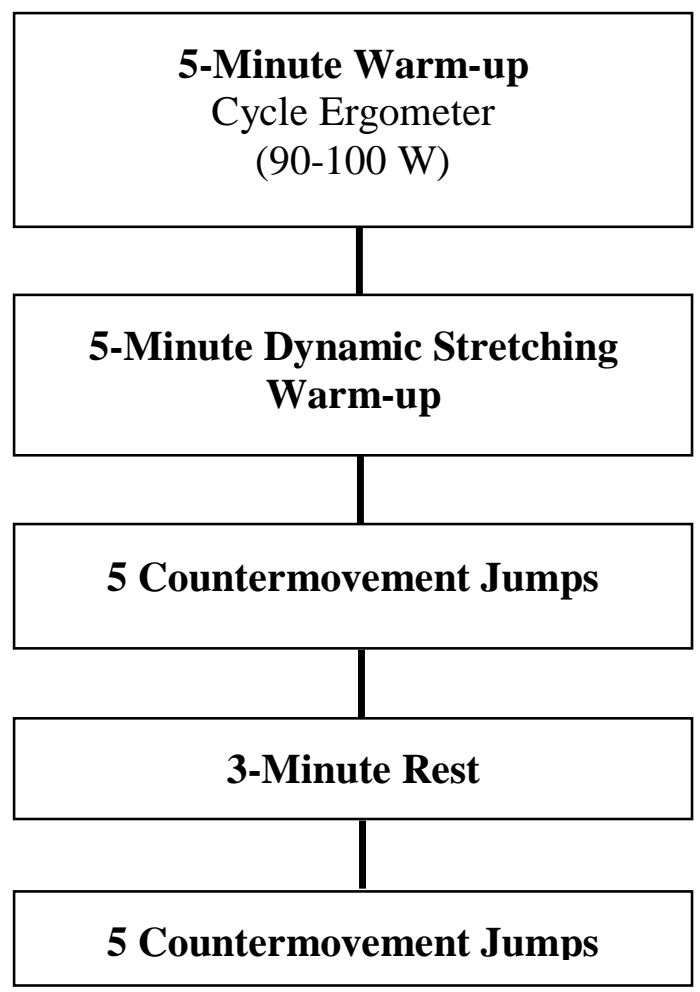


Figure 4.4: Correlation analysis between peak forces from accelerometer and force plate data
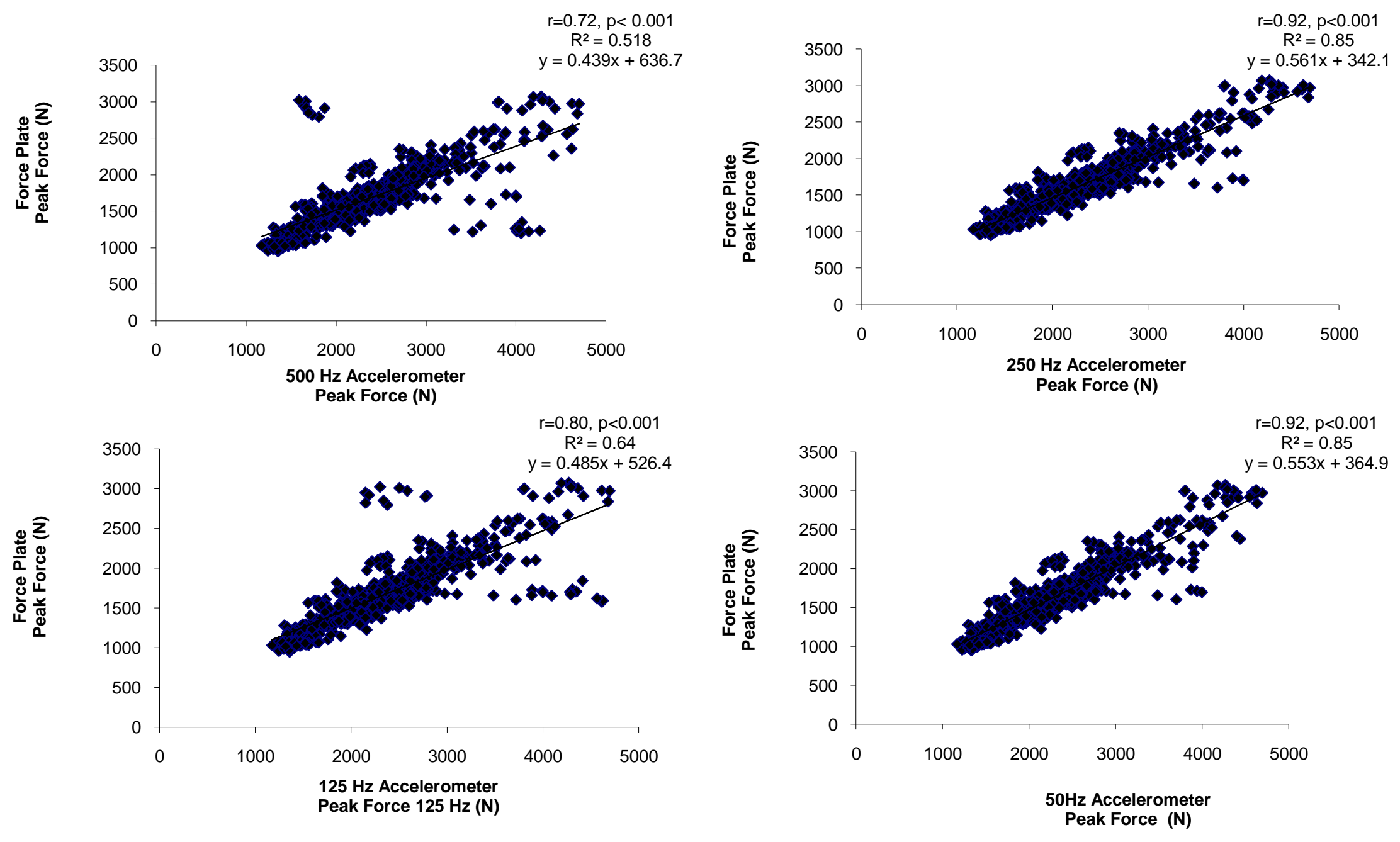
Figure 4.5: Correlation analysis between rate of force development from accelerometer and force plate data.
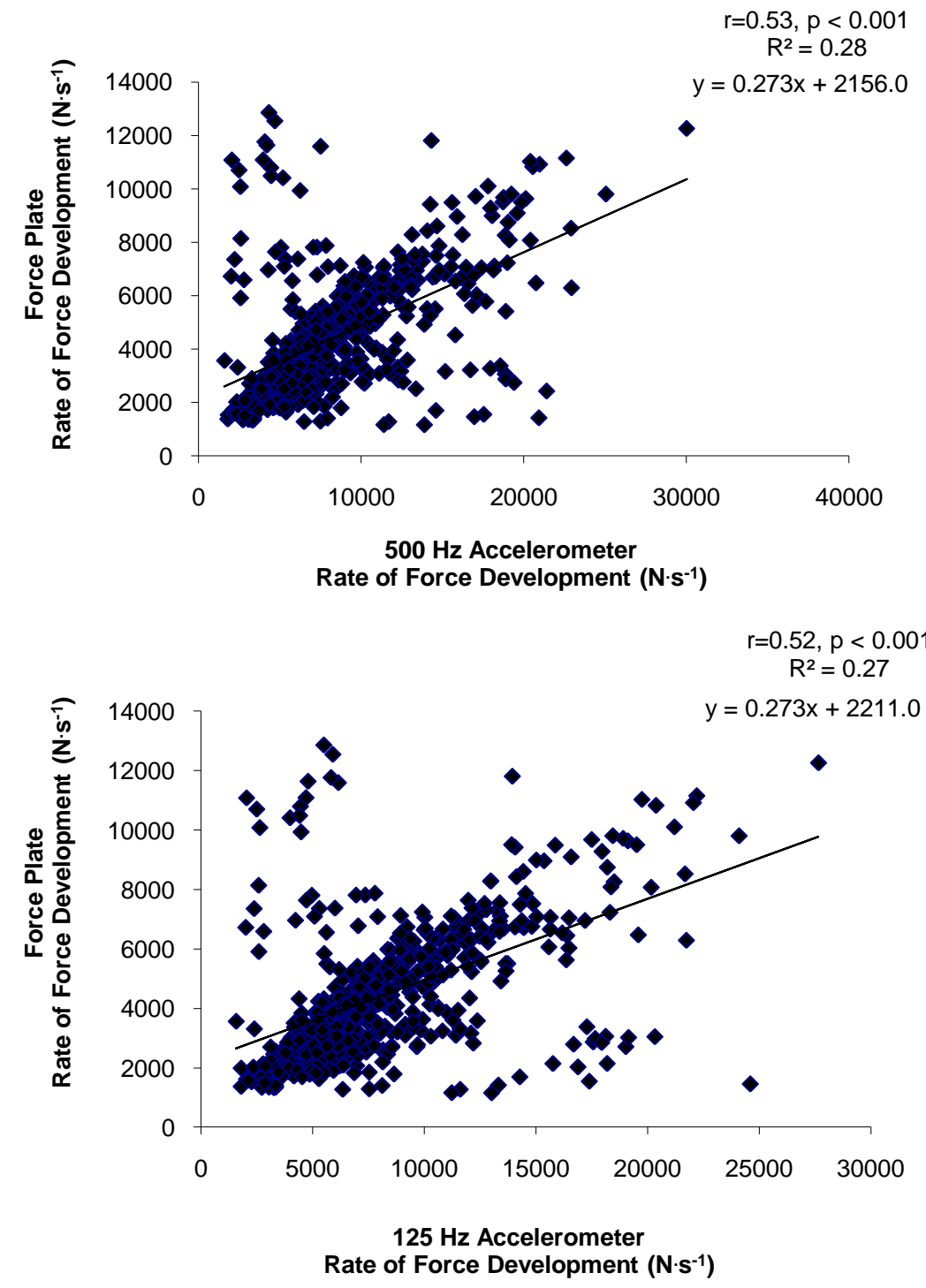
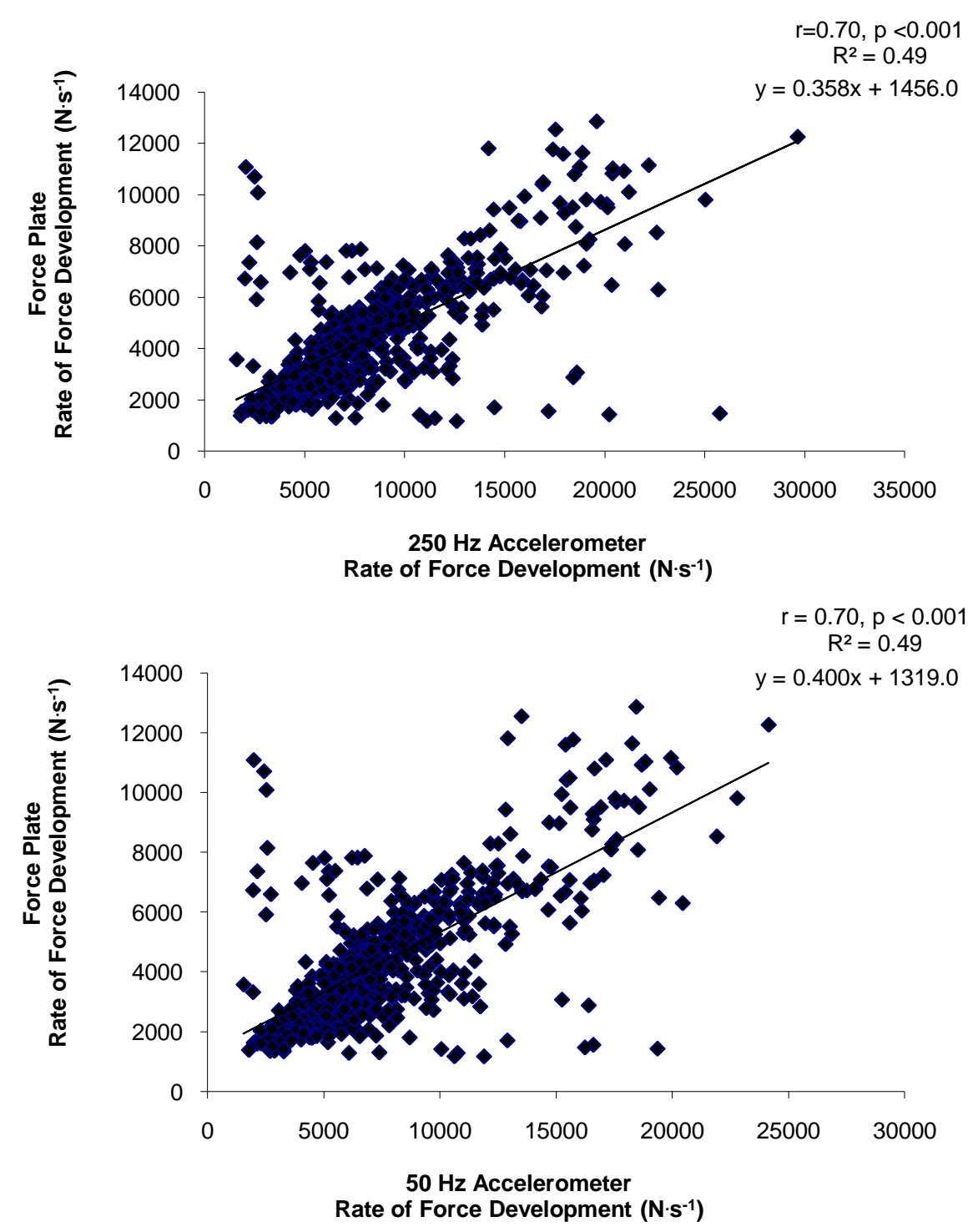
Figure 4.6: Correlation analysis between peak powers from accelerometer and force plate data
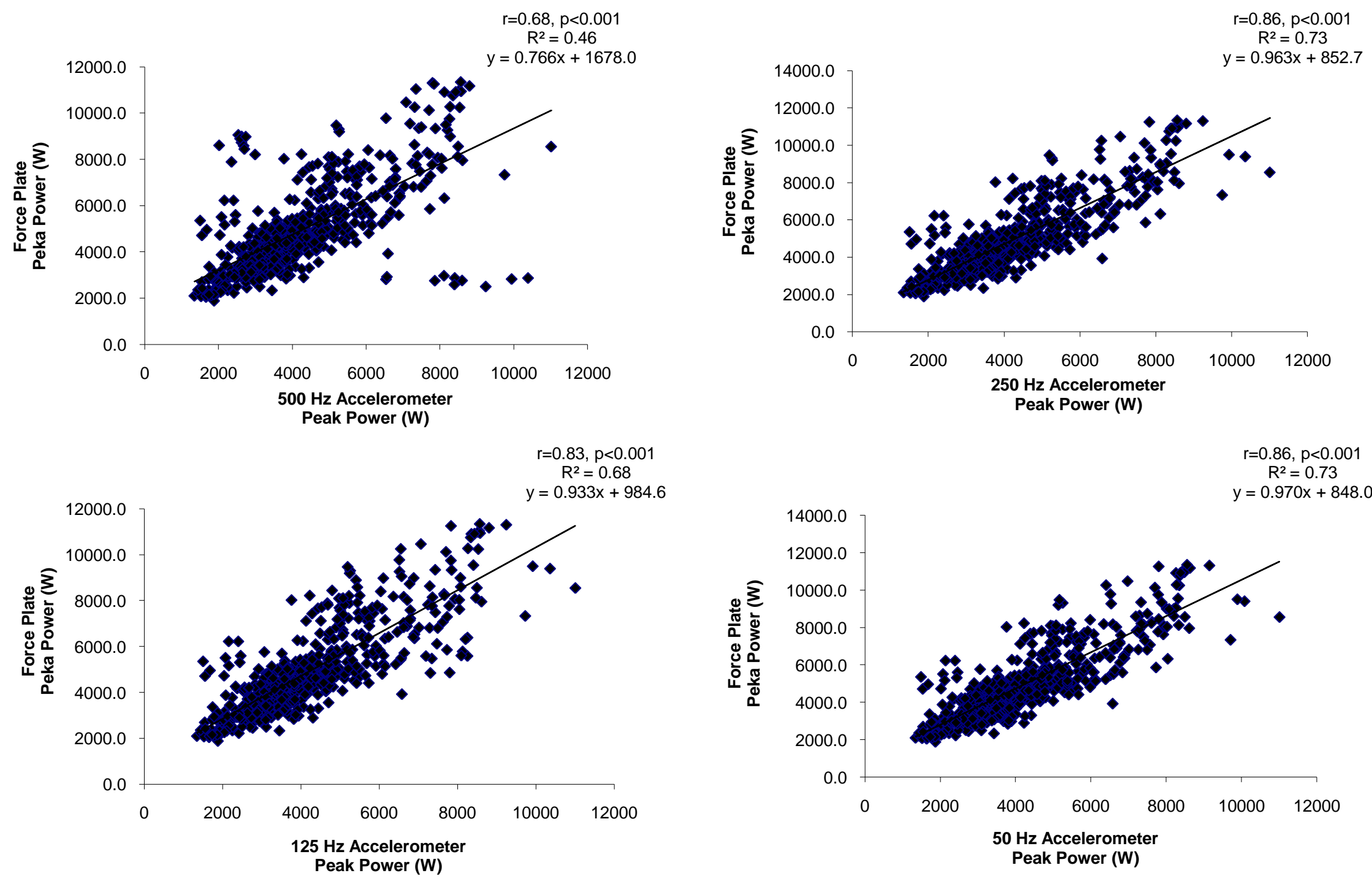
Figure 4.7: Correlation analysis between peak velocities from accelerometer and force plate data.
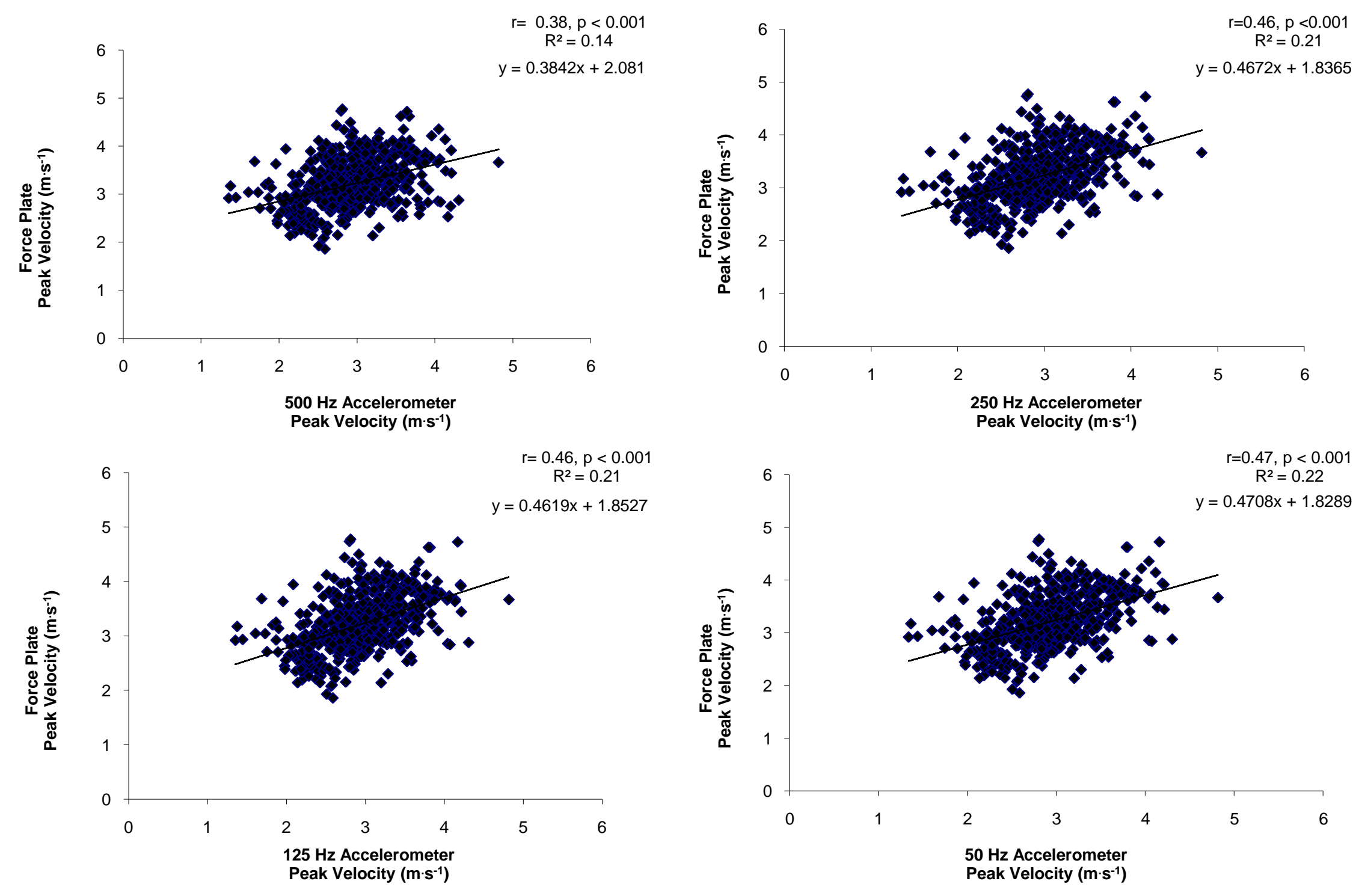
Figure 4.8: Correlation analysis between flight time from accelerometer and force plate data
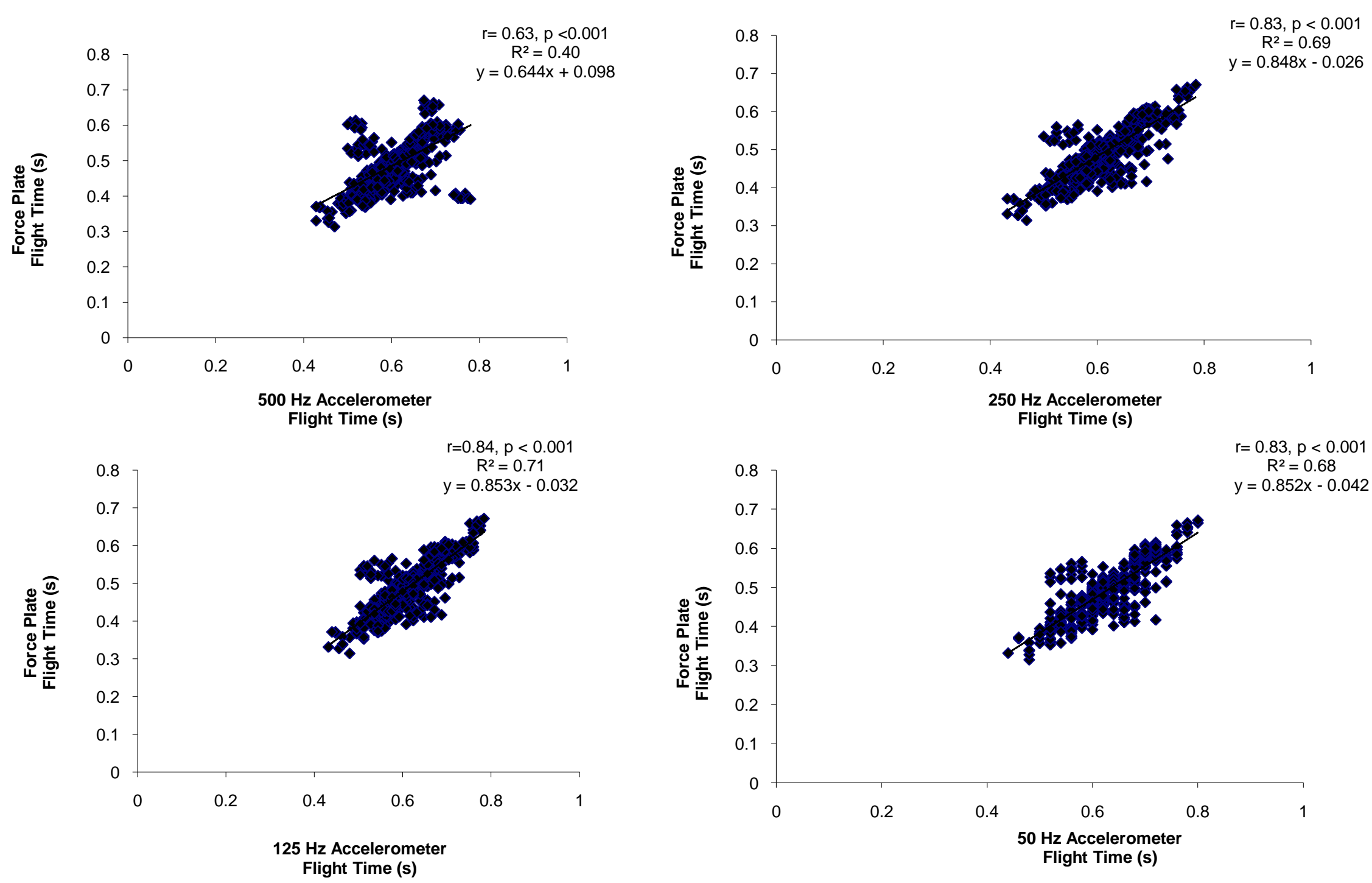
Figure 4.9: Correlation analysis between vertical displacements from accelerometer and force plate data
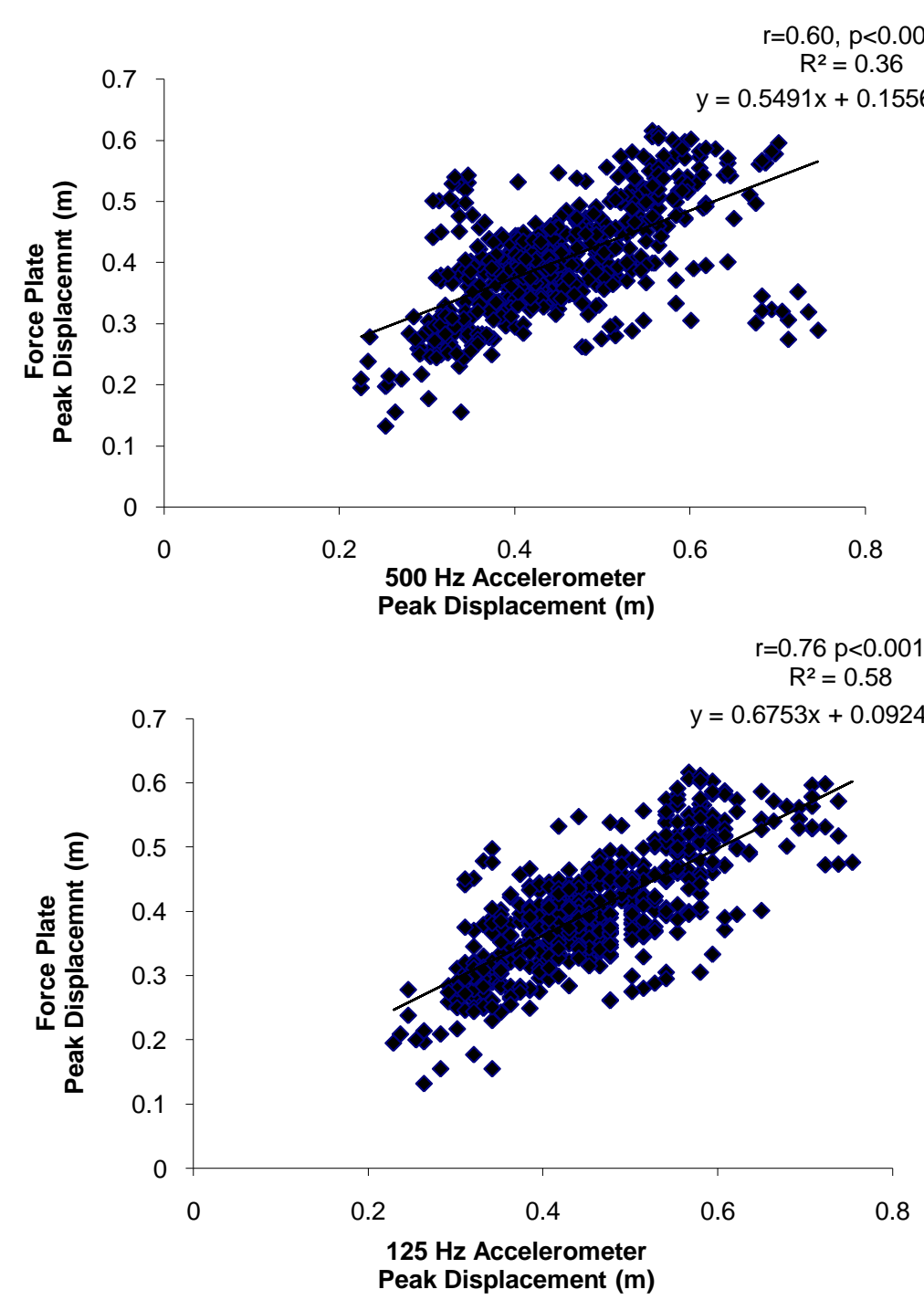
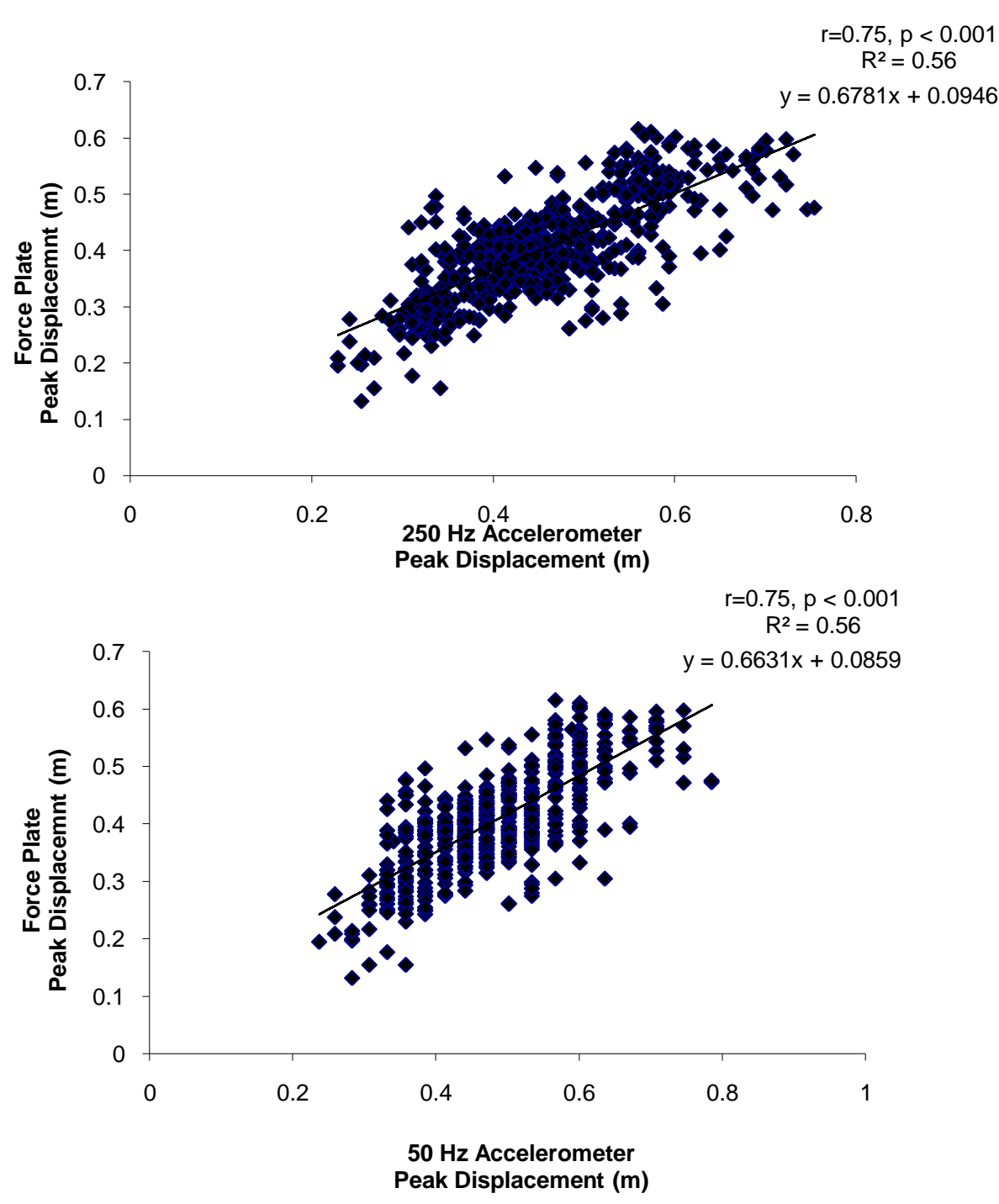
Figure 4.10: Correlation analysis between peak forces from accelerometer and force plate data for the women's sub-group
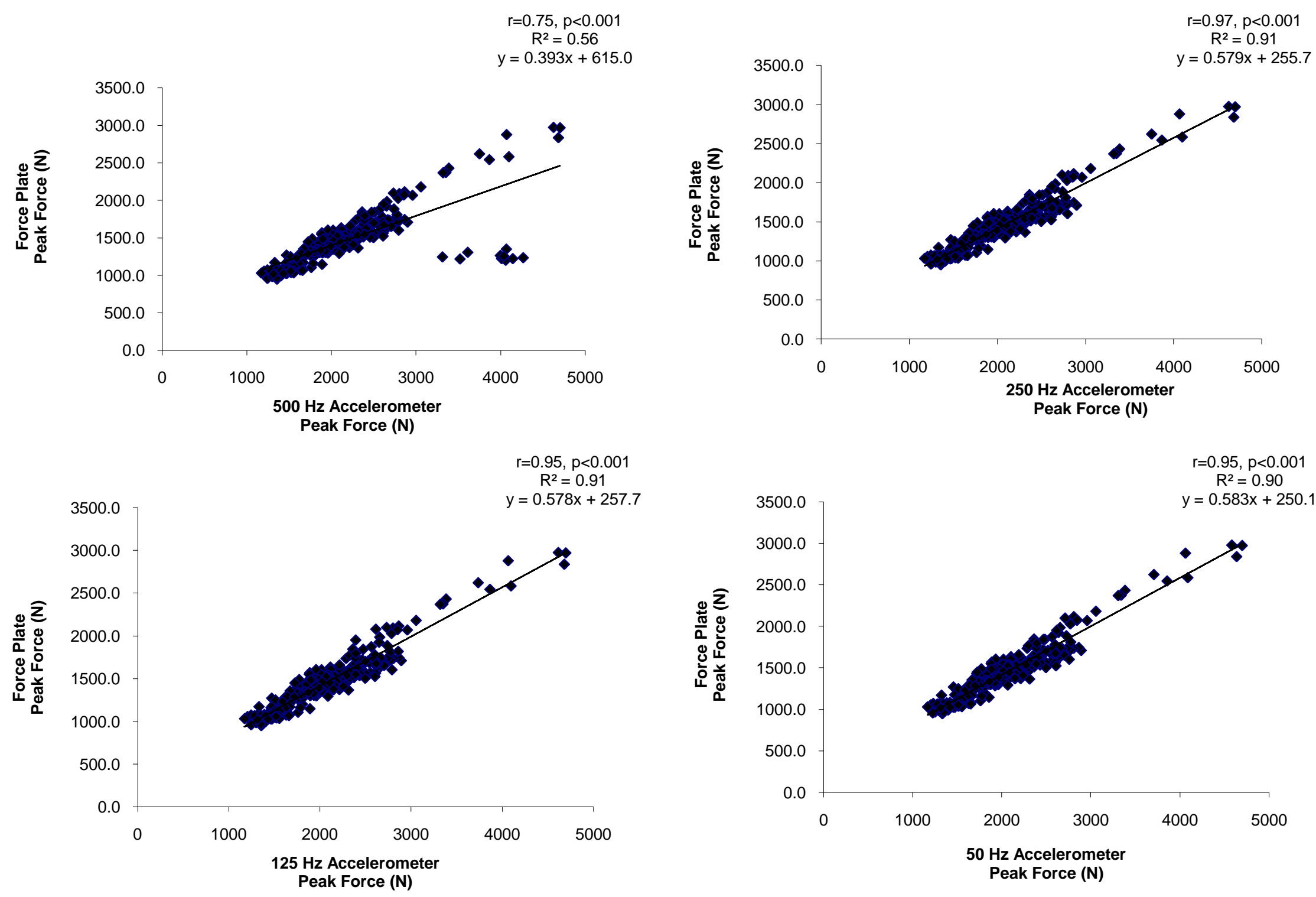
Figure 4.11: Correlation analysis between RFD from accelerometer and force plate data for the women's sub-group
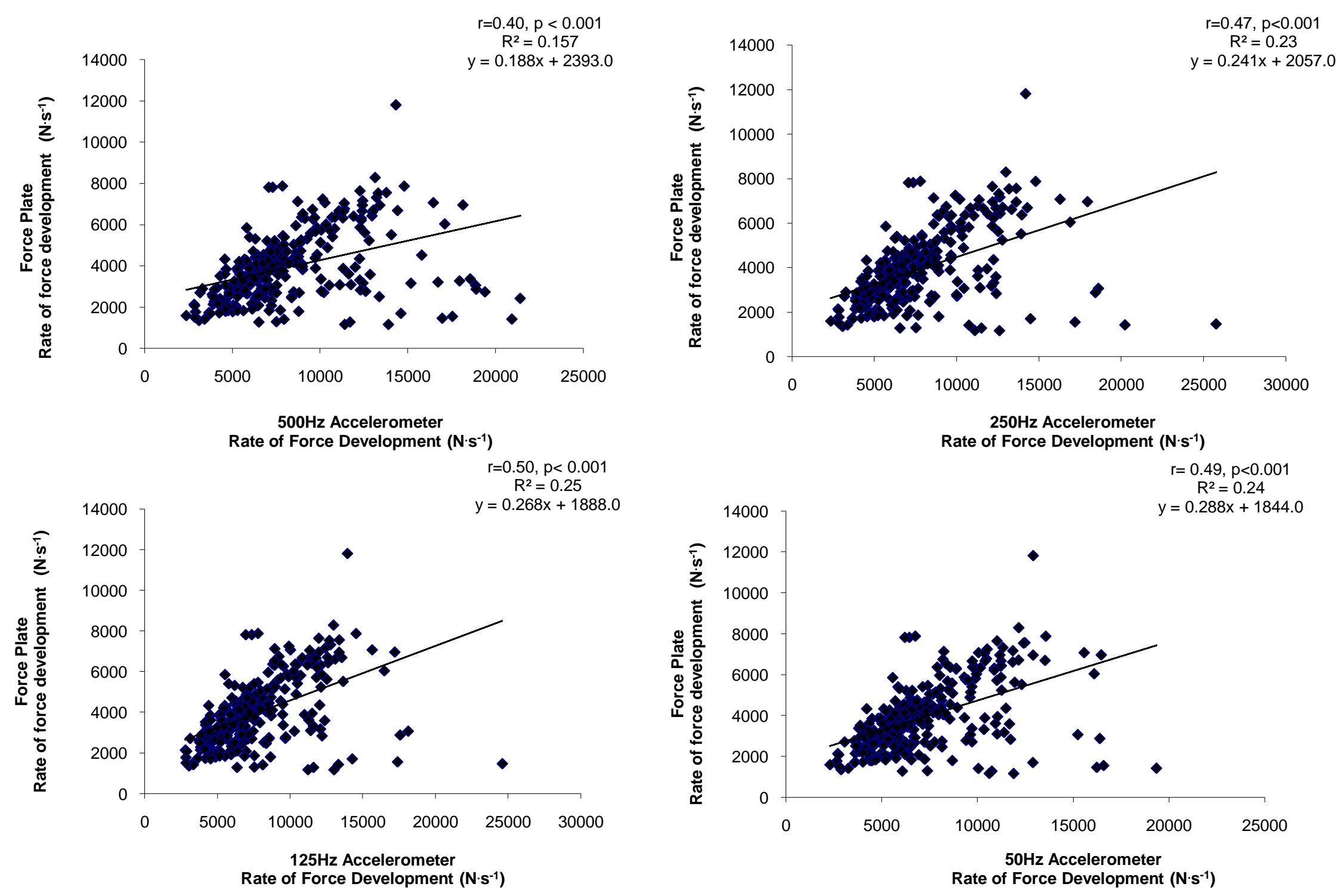
Figure 4.12: Correlation analysis between peak powers from accelerometer and force plate data for the women's sub-group
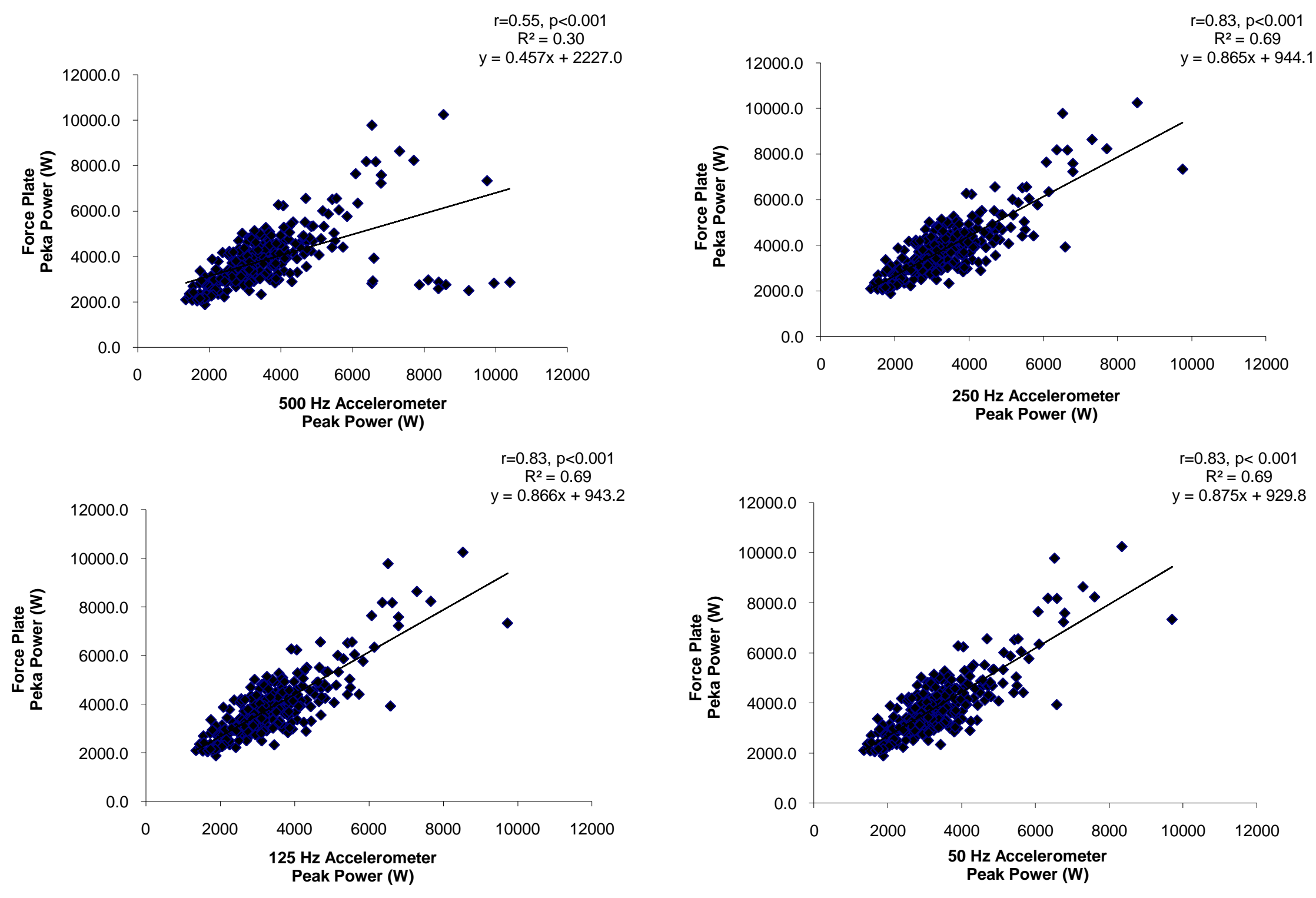
Figure 4.13: Correlation analysis between peak velocities from accelerometer and force plate data for the women's sub-group
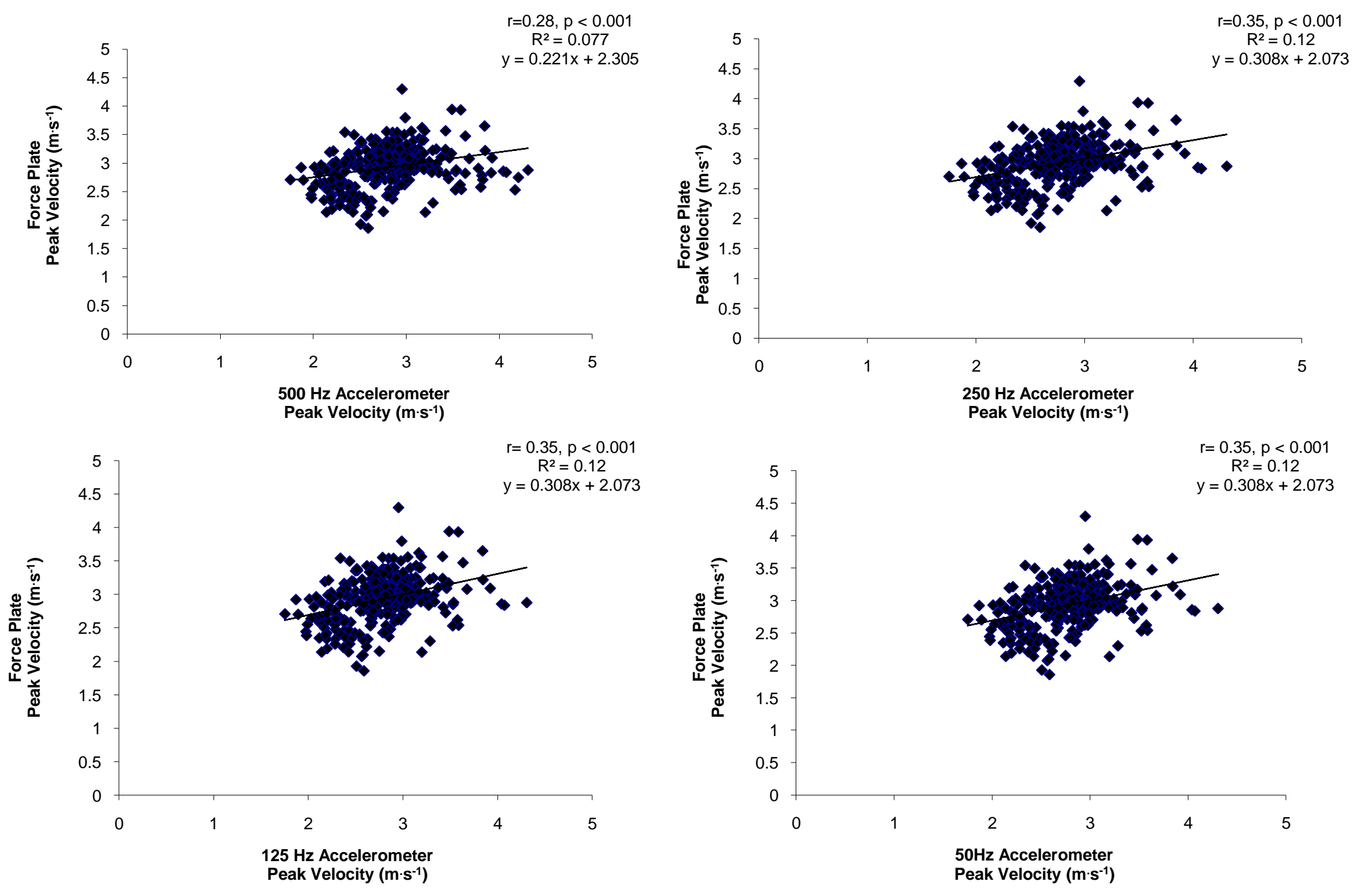
Figure 4.14: Correlation analysis between flight time from accelerometer and force plate data for the women's sub-group
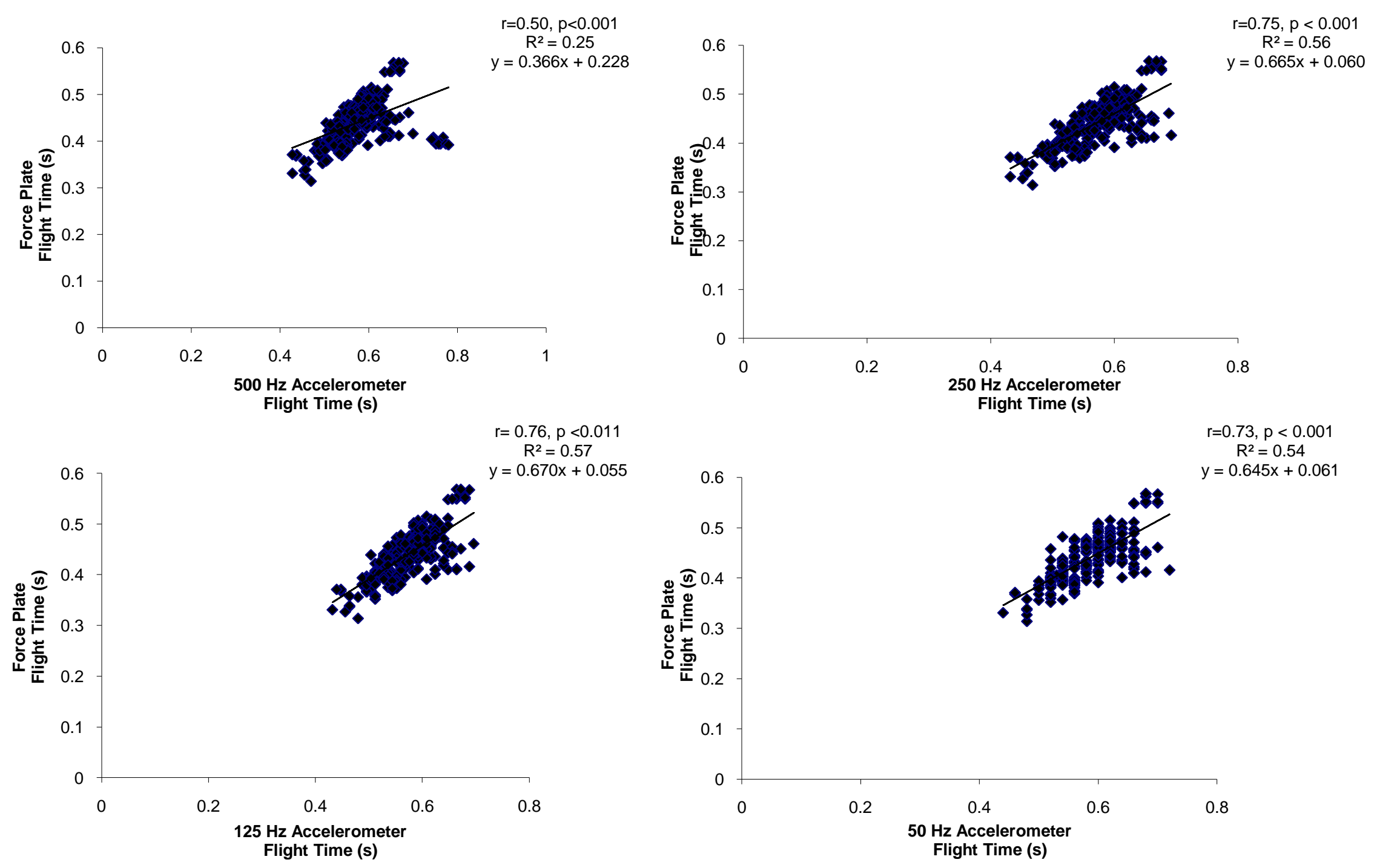
Figure 4.15: Correlation analysis between flight time from accelerometer and force plate data for the women's sub-group
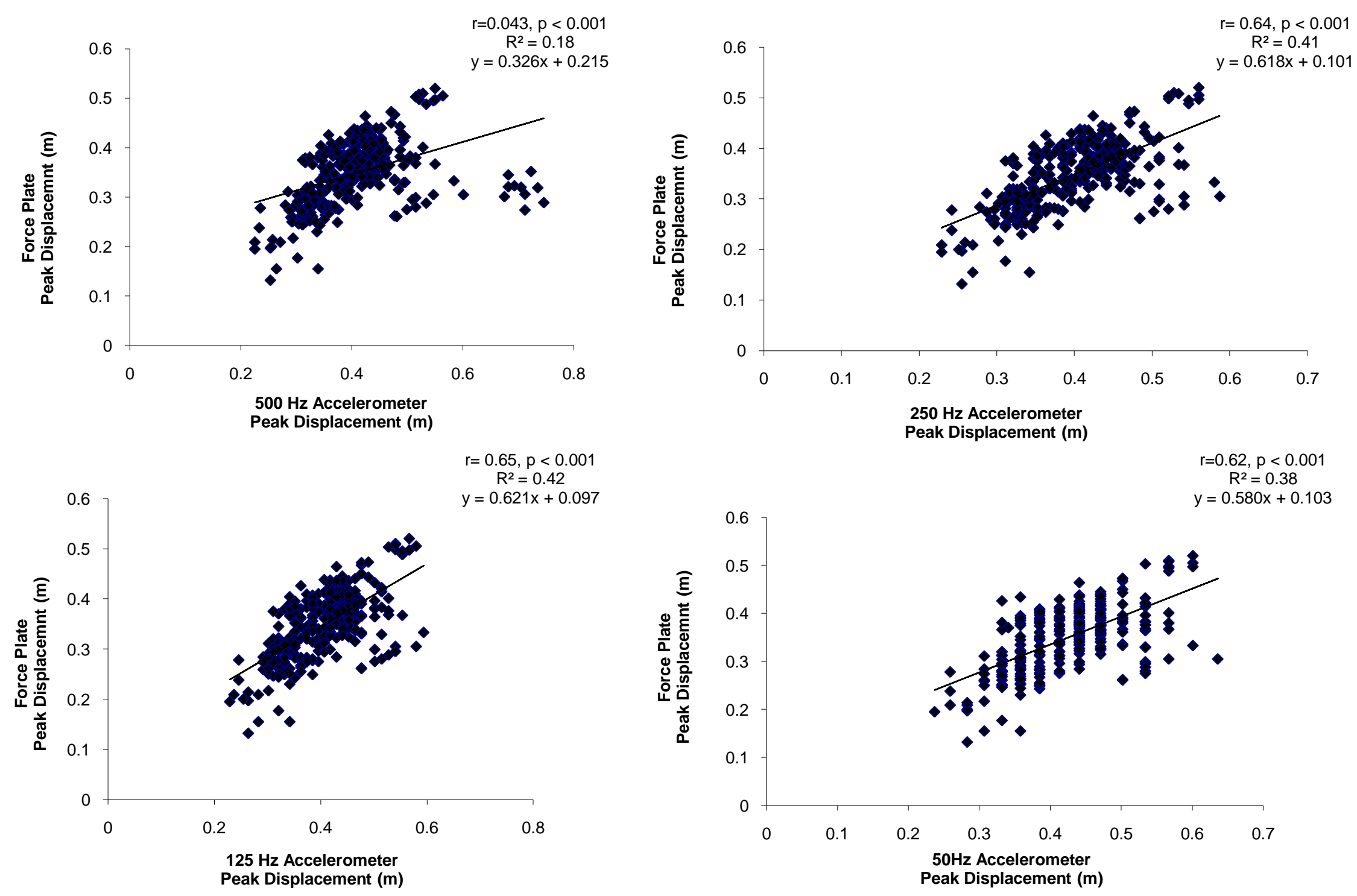
Figure 4.16: Correlation analysis between peak forces from accelerometer and force plate data for the men's sub-group
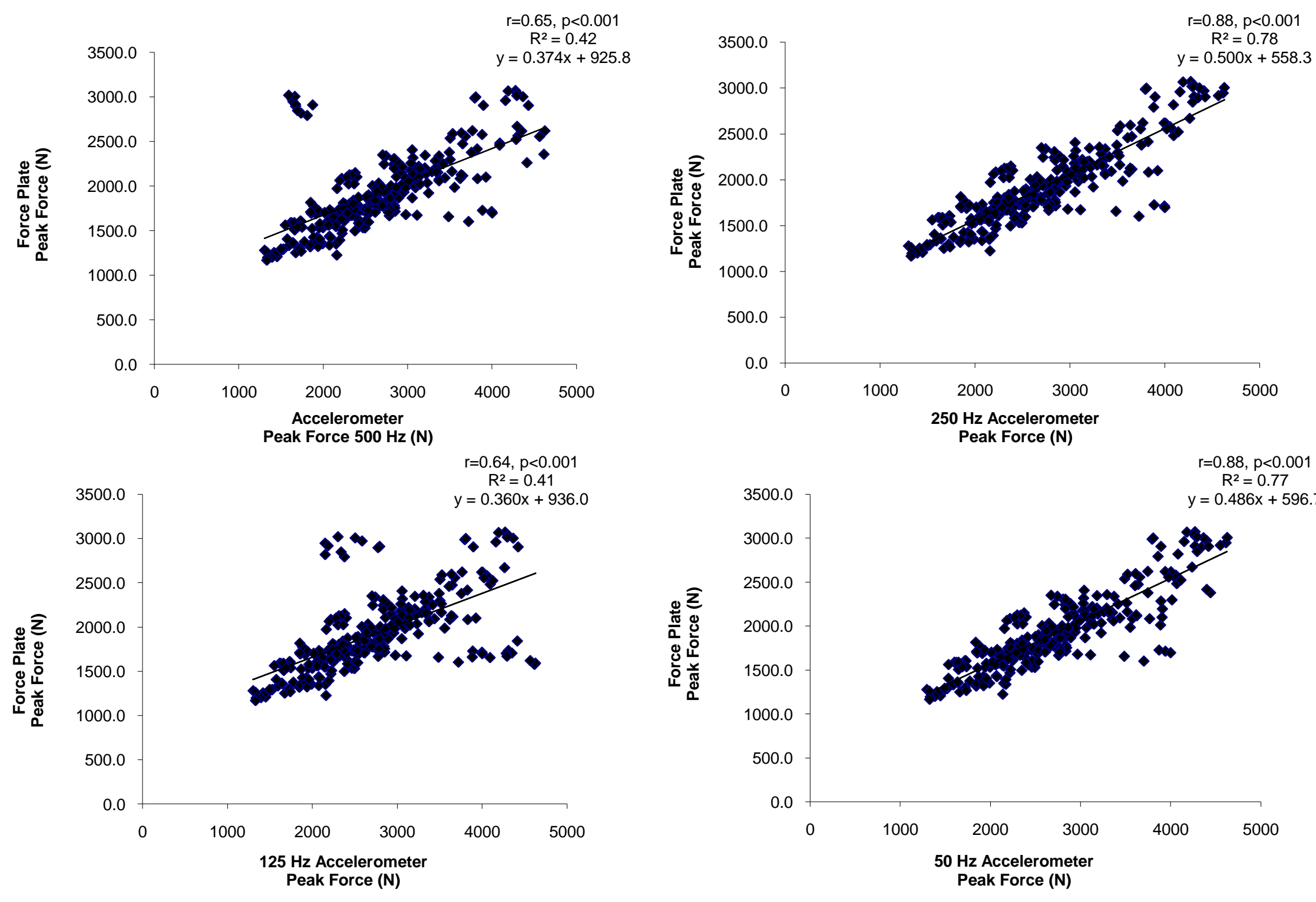
Figure 4.17: Correlation analysis between RFD from accelerometer and force plate data for the men's sub-group

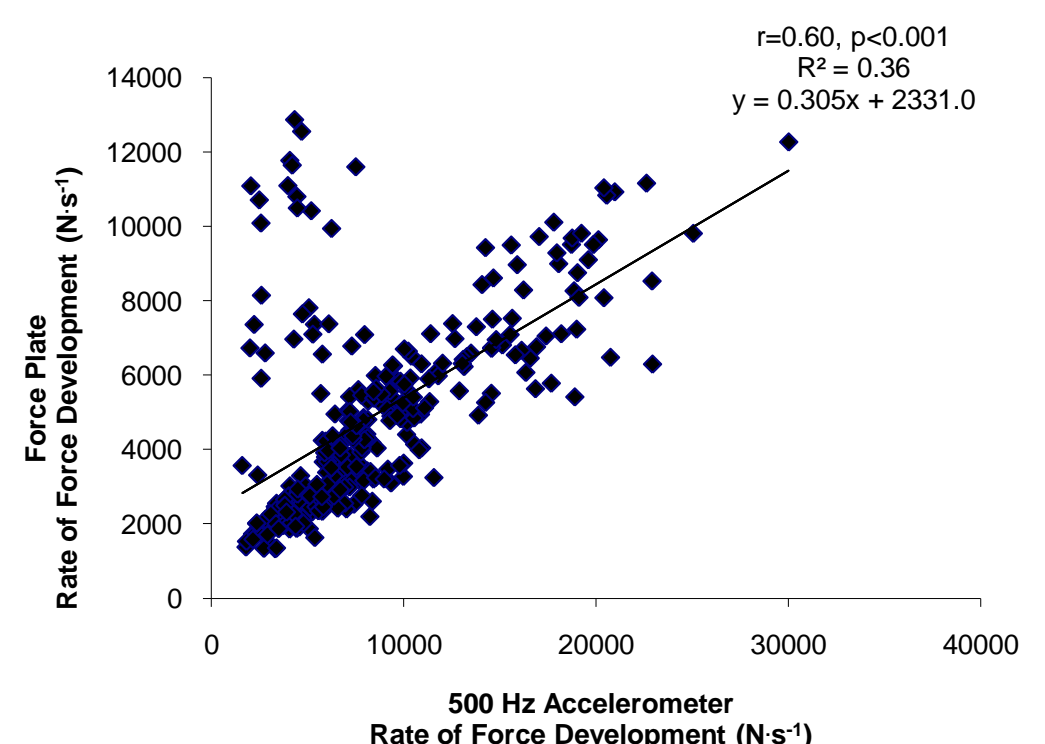

Rate of Force Development $\left(\mathbf{N} \cdot \mathbf{s}^{-1}\right)$

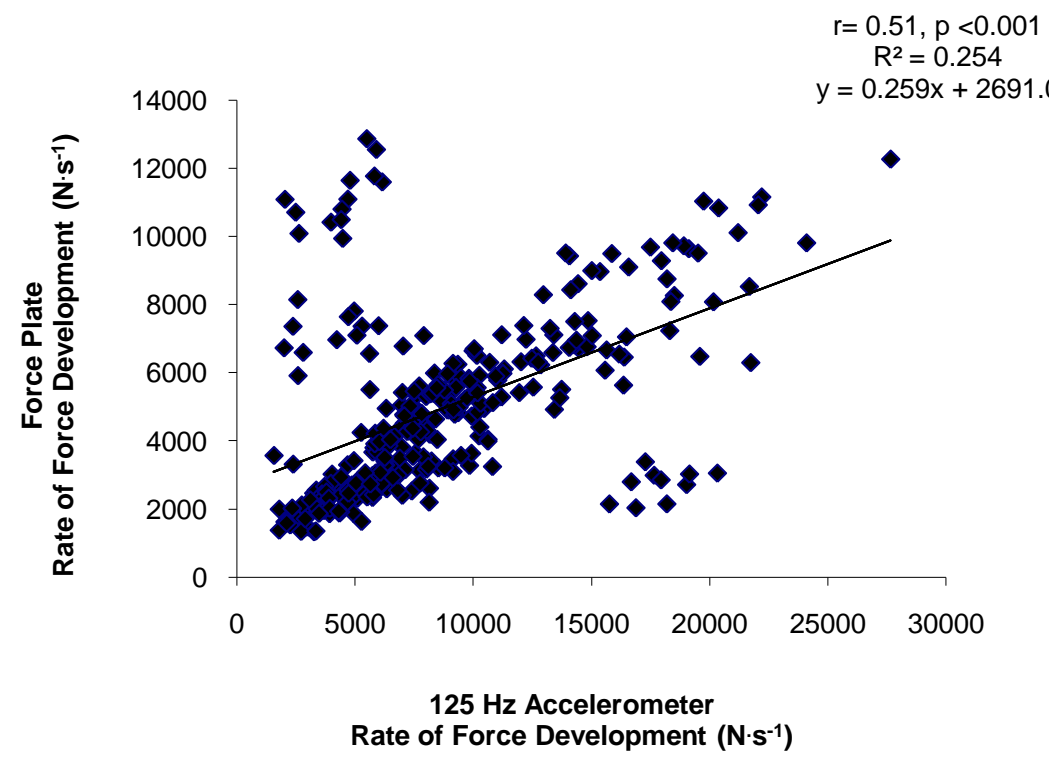

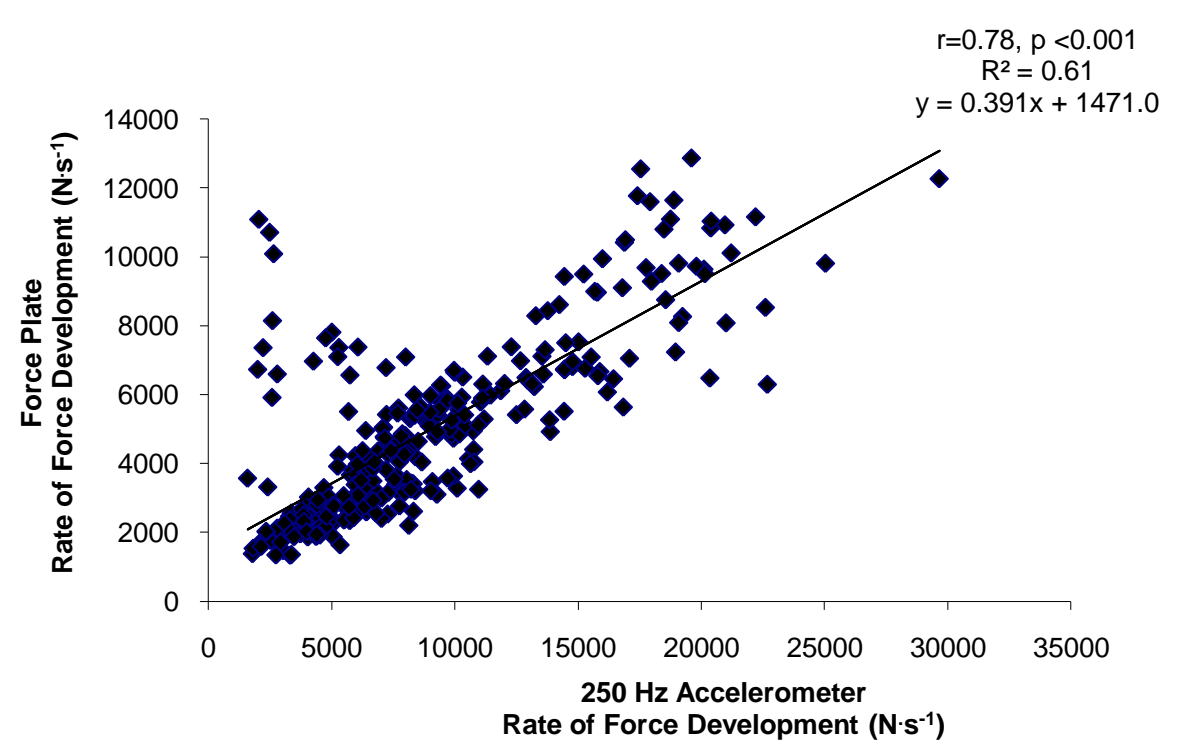

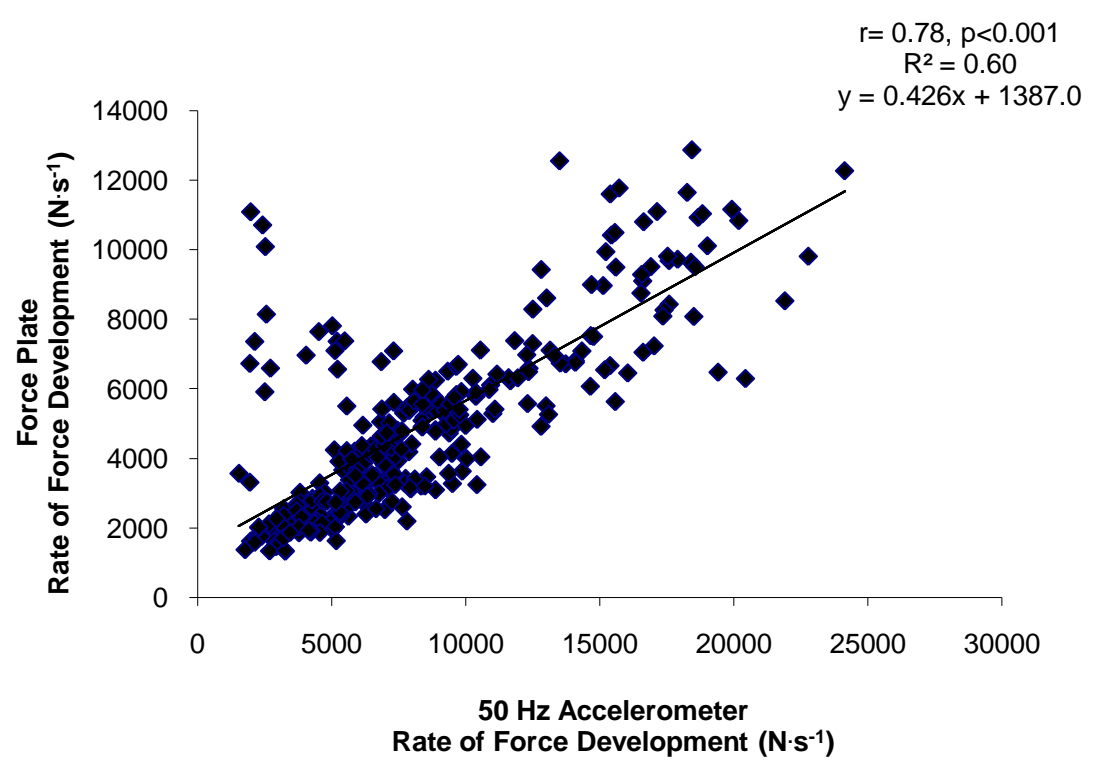


Figure 4.18: Correlation analysis between peak powers from accelerometer and force plate data for the men's sub-group
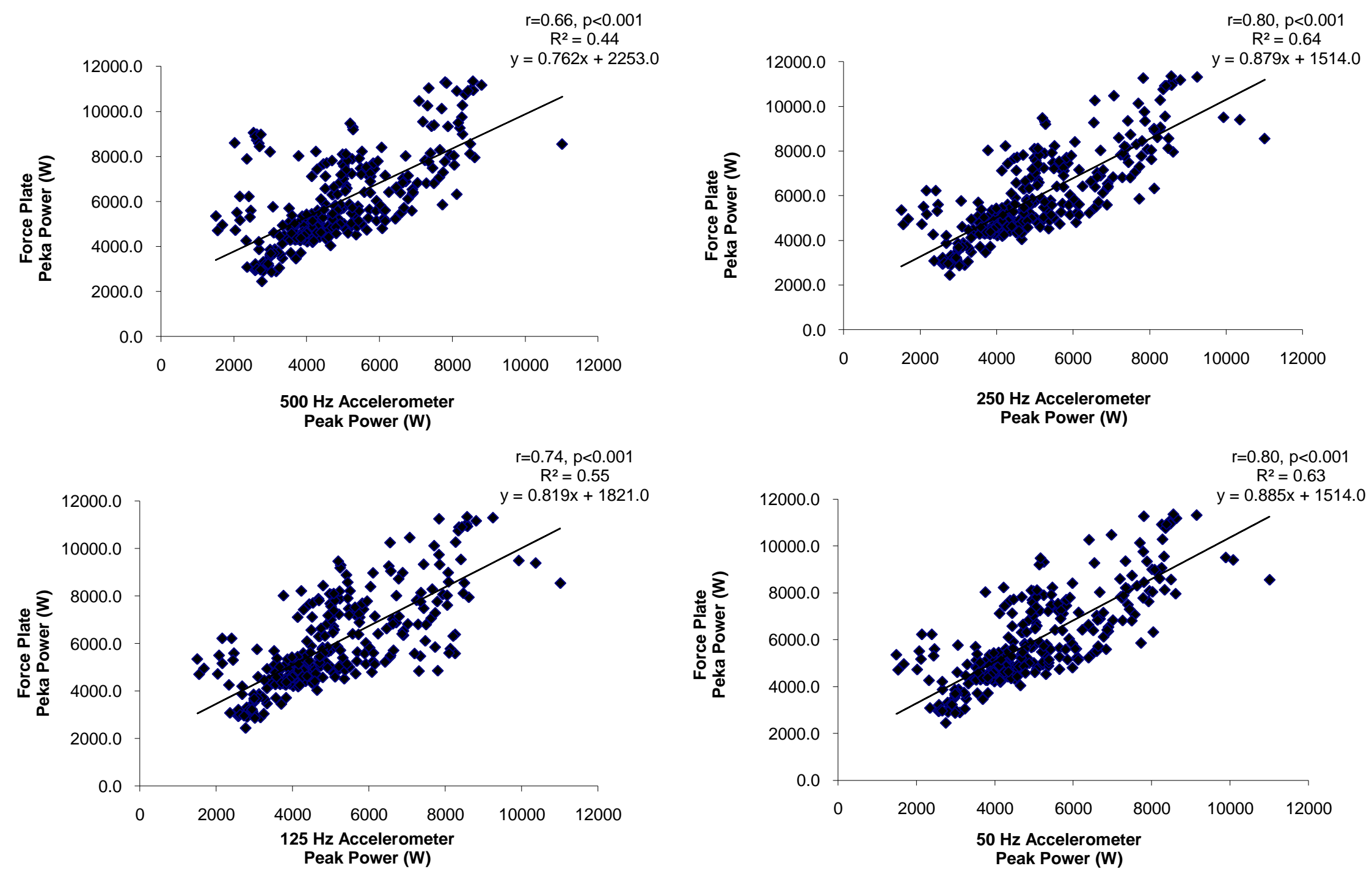
Figure 4.19: Correlation analysis between peak velocities from accelerometer and force plate data for the men's sub-group
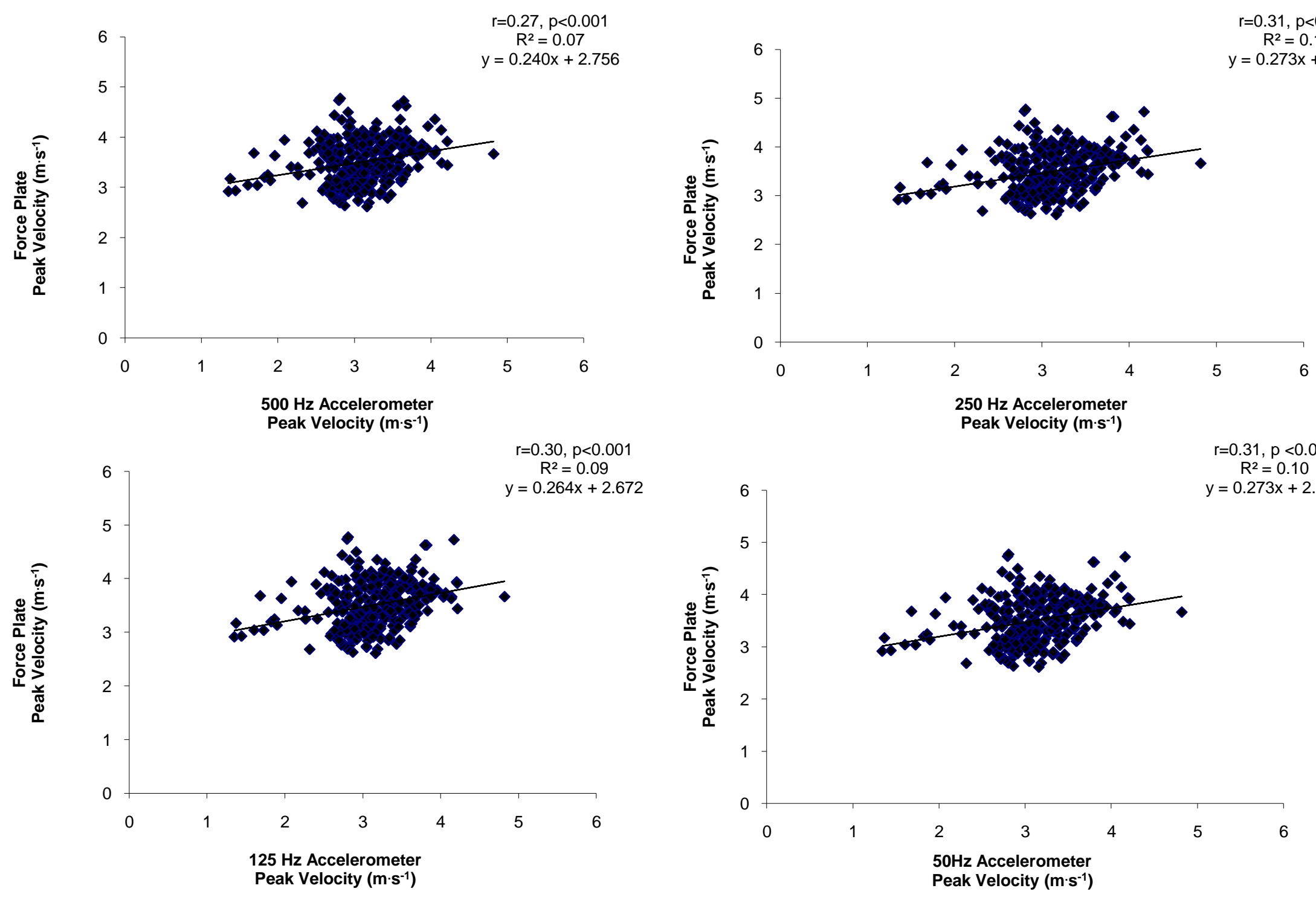
Figure 4.20: Correlation analysis between flight time from accelerometer and force plate data for the men's sub-group
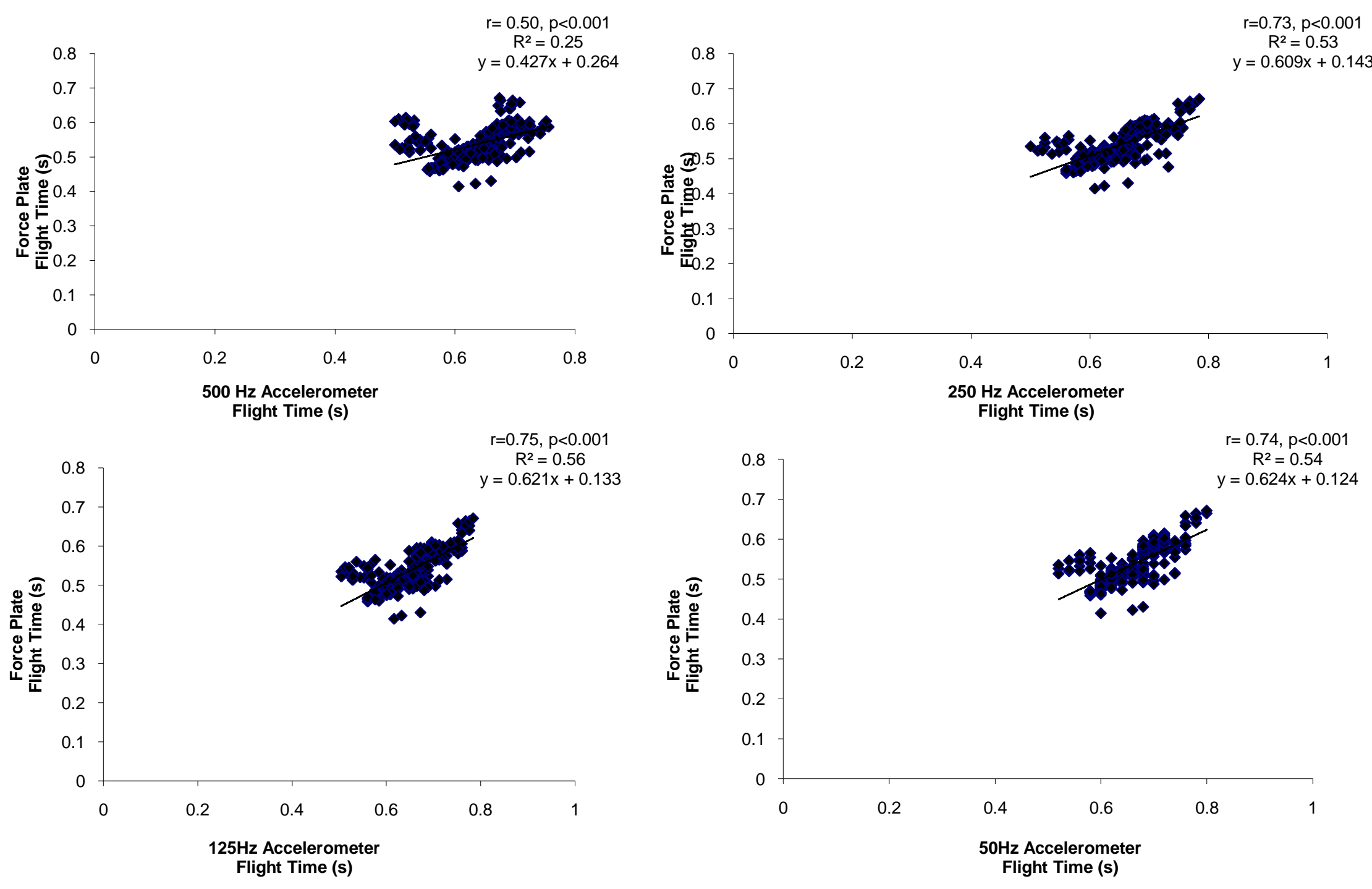
Figure 4.21: Correlation analysis between vertical displacements from accelerometer and force plate data for the men's subgroup
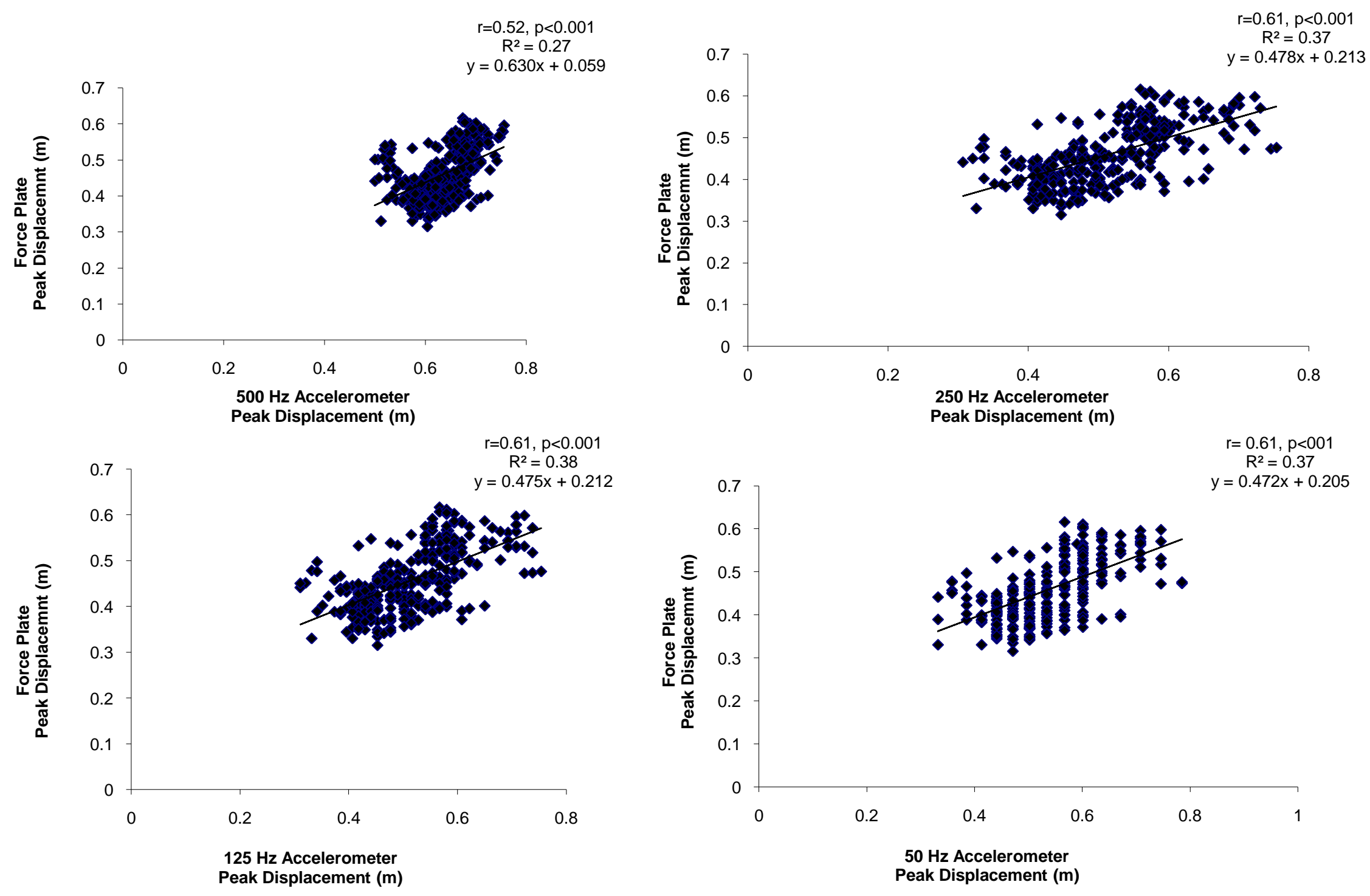
Advertisement:

DO YOU WANT TO KNOW HOW MUCH BODY FAT YOU HAVE?

DO YOU WANT TO KNOW HOW HIGH YOU CAN JUMP?

ARE YOU BETWEEN THE AGES OF 18 AND 30?

ARE YOU INTERESTED IN VOLUNTEERING FOR A RESEARCH STUDY?

If you answered YES to all of the above questions you are invited to participate in a study that will evaluate your lower body strength, body composition, and jumping ability.

WHAT YOU GET FOR PARTICIPATING IN THIS STUDY:

a. BODY COMPOSITION: Your body composition will be assessed with the use of the BOD POD and you will receive information about how much lean body mass and fat mass you have.

b. JUMPING ABILITY: Your ability to perform 5 countermovement and 5 static vertical jumps will be evaluated with the use of a new fitness testing device call the MyoTEST. The MyoTEST was designed in Switzerland and determines the speed at which you jump, how much power you generate, and how fast you accelerate.

In order for you to participate you will have to give your informed consent and answer questions related to your health status and exercise history.

If you participate in this study you will be required to come to the Instructional Laboratory (G276) and the Human Performance Laboratory 2 times over the course of 2 weeks. Appointments will be scheduled so as to best meet your time constraints (i.e. class and work schedule).

ARE YOU INTESTED IN LEARNING MORE? If so please contact:

Dr. G. Gregory Haff C.S.C.S*D, FNSCA

Department of Human Performance and Applied Exercise Science

Division of Exercise Physiology

8313 HSC-S, PO Box 9227

Morgantown, WV 26506-9227

304-293-4299 or ghaff@hsc.wvu.edu

Institutaionl Review Board (IRB) Approval on file

\begin{tabular}{|c|c|c|c|c|c|c|c|c|}
\hline 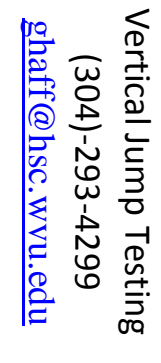 & 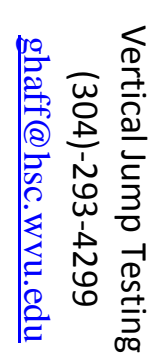 & 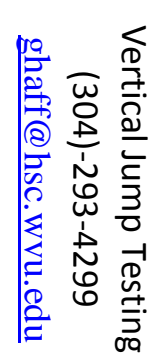 & 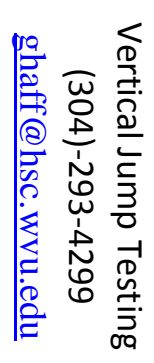 & 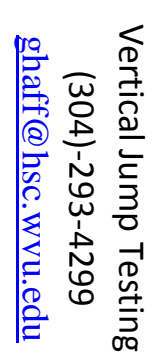 & 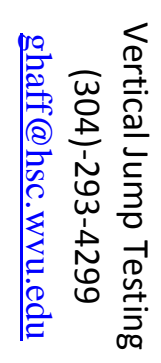 & 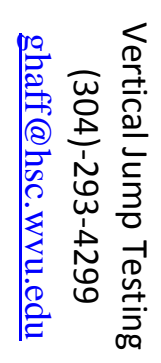 & 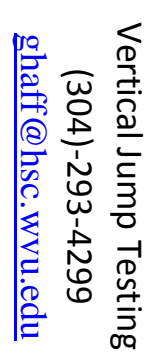 & 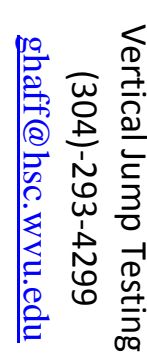 \\
\hline
\end{tabular}




\title{
W. WestVrrginiaUniversity. \\ Office of Research Compliance \\ CONSENT AND INFORMATION FORM
}

\author{
OMR ICF
}

$\begin{array}{ll}\text { Principal Investigator: Haff, Gregory } \\ \text { Department: } & \text { MEDICINE - Exercise Physiology } \\ \text { Tracking Number: } & \text { H-21411 }\end{array}$

\section{Study Title:}

Validation of the use of accelerometers to determine vertical jump power outputs and displacements

\section{Co-Investigator(s):}

Jean McCrory, Ryan Ruben, Michelle Mollinari, Stephanie Burgess, Kelsey Fowler

\section{Sponsor}

\section{Contact Persons}

In the event you experience any side effects or injury related to this research, you should contact Dr. G. Gregory Haff at (304) 293-4299. For more information about this research and about research-related risks or injury, you can contact Dr. G. Gregory Haff at (304)293-4299.

For information regarding your rights as a research subject, you may contact the Office of Research Compliance at 304/293-7073.

\section{Introduction}

You, have been asked to participate in this research study, which has been explained to you by . This study is being conducted by G. Gregory Haff, Ph.D. in the Department of

Tracking \#: H-21411

Approved On: 01/02/2009

Valid Through: 01/01/2010

Last Amended: 1/27/2009
Page 1 of 6

$$
\overline{\text { Initials }} \quad \overline{\text { Date }}
$$


Human Performance and Applied Exercise Science at West Virginia University.

\section{Purposes of the Study}

The purpose of this study is to determine if a new computer sensing device, known as an accelerometer, can accurately measure how high a person can jump. In this study two series of jumping activities will be measured by this computer sensor. The first will require you to squat down and jump vertically as fast as possible. The second will require you to squat down and hold the bottom of the squat for 3 seconds and then jump as high as possible. The data collected by the new computer sensing device will be compared to data collected by a specialized scale, known as a force plate, which can determine how high and fast you jump. The information gained from this study will be used to adapt the accelerometer technology. WVU expects to enroll approximately 200 subjects (100 men and 100 women) in this investigation.

\section{Description of Procedures}

This study will be done in the West Virginia University School of Medicine Human Performance Laboratory and the Instructional Laboratory. The study involves an assessment of body weight and body composition (Body fat and lean body mass), and vertical jumping ability. You will be required to attend two testing sessions. The total time requirements for the two sessions if you participate in this investigation will be approximately 3 hours.

Testing Session \# 1: This session should take between 1.5 to 2 hours for you to complete.

Health and Training History: You will be asked to fill out a questionnaire regarding your health/medical history and your exercise or strength training history. This will take approximately 15-20 minutes for you to complete. You do not have to answer all the questions. You will have the opportunity to see the questionnaire before signing this consent form.

Measurement of your height, weight and body composition: You will be required to wear a bathing suit when having your body weight and body composition measured. Your body weight will be measured without shoes using a calibrated scale, while your height will be measured with a

Tracking \#:

Approved On:

Valid Through:

Last Amended:
$\mathrm{H}-21411$

01/02/2009

01/01/2010

$1 / 27 / 2009$
Page 2 of 6

$\overline{\text { Initials }} \overline{\text { Date }}$ 
stadiometer. Your body composition will be estimated in a small chamber called the BodPOD. You will sit in an enclosed chamber. The door will be shut and you will rest comfortably. This machine measures the amount of air that your body pushes out of the chamber and it can determine the amount of fat and lean body mass on your body from this measurement. You will be in your bathing suit during this test. This test will take approximately $2-3$ minutes to complete.

Familiarization with Jumping Protocol: After completing the assessment of body composition you will be familiarized with the jumping protocol which will be used in this study. The jumping protocol will require you to perform a standardized warm-up consisting of cycling for 5 minutes followed by 5 minutes of dynamic movement exercises, such as marching. After warming up you will rest for 5 minutes and then perform a series of 5 vertical jumps in which you squat down and jump as quickly as possible (countermovement vertical jump). After completing these jumps you will rest for 5 minutes. After resting for 5 minutes you will perform a series of 5 vertical jumps in which you squat down and pause for 3 seconds and then jump as high as possible (static vertical jump). Instruction will be given by a certified strength and conditioning specialist on how to perform the various jumping activities. You will be given time to practice this activity so as to maximize your jumping performance.

Session \#2: There will be 7 days between each of these sessions and each session should take approximately 1 hour. You will be randomly assigned to a testing order for the session. The order of the vertical jump tests will be randomly assigned but the basic procedure for testing will be similar to the familiarization session.

Jumping session: As with the initial session you will perform 5 minutes of cycling followed by 5 minutes of dynamic stretching, such as marching. After completing the warm-up portion of the session you will rest for 5 minutes and be fitted with a computer sensor (accelerometer). You will then perform 5 vertical jumps (one of the jumping protocols practiced in the familiarization session: either countermovement or static depending upon which testing order group you are assigned to). After completing the first series of 5 jumps you will rest for 5 minutes. After resting for 5 minutes you will then perform 5 vertical jumps (either countermovement or static depending upon depending upon which testing order group you are assigned to).

Tracking \#: H-21411

Approved On: 01/02/2009

Valid Through: 01/01/2010

Last Amended: 1/27/2009
Page 3 of 6

$\overline{\text { Initials }} \overline{\text { Date }}$ 


\section{Risks and Discomforts}

Body Composition: There are generally no risks associated with the measurement of body composition. However, if you are claustrophobic you may feel uncomfortable in the BOD PODOे Chamber. Body Composition will also be estimated from skinfold assessment. The skinfold assessment may cause a minimal discomfort from the pinching action used to measure the skinfold.

Jumping Activity: This test is used to assess jumping performance and is considered to impose minimal risks. The most common risk associated with this test is minor muscle soreness, which can last for a day or two. In very rare instances muscle strains, fainting and abnormal blood pressure responses can occur. In order to insure your safety you will be monitored continuously by a certified strength and conditioning specialist.

\section{Alternatives}

You do not have to participate in this study.

If you are uncomfortable with the BOD PODÒ chamber you can chose to only participate in a skinfold body composition analysis procedure. You do not have to participate in any part of this investigation.

\section{Benefits}

This data will help provide researchers and sports scientists an understanding about the effectiveness of accelerometer based vertical jump testing methodologies. Additional benefits from your participation will include a detailed report on your body composition and vertical jump ability. This information may be used to get a better understanding of your overall health and wellness. The information gathered from this investigation may lead to improved implementation of vertical jump testing methods in clinical and athletic sessions.

Tracking \#: H-21411

Approved On: 01/02/2009

Valid Through: 01/01/2010

Last Amended: 1/27/2009
Page 4 of 6

$\overline{\text { Initials }} \overline{\text { Date }}$ 


\section{Financial Considerations}

You may wish to consult your insurance carrier prior to entering this study. There are no special fees for participating in this study. You will incur the costs of travel to the Health Science Center of West Virginia University School of Medicine for all of the testing sessions. There are no special fees for participating in this study, but any expense associated with injury or treatment of side effects will be billed to you or your insurance company. If you are injured as a result of this research, treatment will be available. Responsibility for this treatment will be borne by you and your insurance company. Compensation for your injuries will not be provided voluntarily by the investigator, sponsor, West Virginia University, or other associated affiliates.

\section{Confidentiality}

Any information about you that is obtained as a result of your participation in this research will be kept as confidential as legally possible. Your research records and test results, just like hospital records, may be subpoenaed by court order or may be inspected by federal regulatory authorities without your additional consent. In addition, there are certain instances where the researcher is legally required to give information to the appropriate authorities. In any publications that result from this research, neither your name nor any information from which you might be identified will be published without your consent

\section{Voluntary Participation}

Participation in this study is completely voluntary. You are free to withdraw your consent or refuse to participate in this study at any time. Refusal to participate or withdrawal will not affect your future care at West Virginia University or your class standing or grades, and will involve no penalty to you. In the event new information becomes available that may affect your willingness to participate in this study, this information will be given to you so that you can make an informed decision about whether or not to continue your participation. You have been given the opportunity to ask questions about the research, and you have received answers concerning areas you did not understand.

Tracking \#: H-21411

Approved On: 01/02/2009

Valid Through: 01/01/2010

Last Amended: 1/27/2009
Page 5 of 6

$$
\text { Initials }
$$

Date 
Upon signing this form, you will receive a copy.

I willingly consent to participate in this research.

Signature of Subject or Printed Name

Date Time

Subjects Legal Representative

The participant has had the opportunity to have questions addressed. The participant willingly agrees to be in the study.

Signature of Investigator or

Printed Name

Date

Time

Co-Investigator

Tracking \#: H-21411

Approved On: 01/02/2009

Valid Through: 01/01/2010

Last Amended: 1/27/2009
Page 6 of 6

$\overline{\text { Initials }} \overline{\text { Date }}$




\section{WestVirginiaUniversity.}

Office of Research Compliance

HIPPA Form

Principal Investigator: Haff, Gregory

Department: MEDICINE - Exercise Physiology

Tracking Number: $\quad \mathrm{H}-21411$

\section{Study Title:}

Validation of the use of accelerometers to determine vertical jump power outputs and displacements

\section{Co-Investigator(s):}

„Jean McCrory, Ryan Ruben, Michelle Molinari, Stephanie Burgess, Kelsey Fowler

\section{Sponsor}

Subject's Name:

ID

Number:

We know that information about you and your health is private. We are dedicated to protecting the privacy of that information. Because of this promise, we must get your written authorization (permission) before we may use or disclose your protected health information or share it with others for research purposes. This form gives that permission. It also helps us make sure that you are correctly told how this information will be used or disclosed. Please read the information below carefully before signing this form. Please ask any questions you may have about this form or its uses. You can decide to sign or not to sign this authorization form. However, if you choose not to sign this authorization form, you will not be able to take part in the research study. Whatever choice you make about this research study, it will not have an effect on your access to medical care.

USE AND DISCLOSURE COVERED BY THIS AUTHORIZATION

DO NOT SIGN A BLANK FORM. You or your authorized representative

\begin{tabular}{lll}
\hline Tracking \#: & $\mathrm{H}-21411$ & Page 1 of 4 \\
Approved On: & $01 / 02 / 2009$ & \\
Valid Through: & $01 / 01 / 2010$ & \\
Last Amended: & $1 / 27 / 2009$ &
\end{tabular}

$\overline{\text { Initials }} \overline{\text { Date }}$


should thoroughly read the information below before signing this form. Who will disclose, receive, and/or use the information? This form will authorize the following person(s), class(es) of persons, and/or organization (s) to disclose, use, and receive the information*:

The research site(s) carrying out this study. This includes UHA or UHA Affiliated, WVU, WVU Hospitals. It also includes each site's research staff and medical staff.

Health care providers who provide services to you as part of this research study.

Laboratories and other people and groups that look into your health information as part of this study in agreement with the study protocol. The United States Department of Health and Human Services (which includes the National Institutes of Health, Food and Drug Administration (FDA)) and other groups that have the right to use the information as required by law.

* If, during the course of the research, one of the companies or institutions listed above merges with, or is purchased by, another company or institution, this authorization to use or disclose protected health

information in the research will extend to the successorcompany or institution.

What information will be used or disclosed?

Information regarding past medical history of cardivascular disease and musculoskeletal joint injuries.

\section{SPECIFIC UNDERSTANDINGS}

By signing this research authorization form, you give permission for the use and/or disclosure of your protected health information described above. The purpose for the uses and disclosures you are authorizing is to carry out the research study explained to you during the informed consent process. It is also to ensure that the information relating to the research is available to all parties who may need it for research purposes. Your protected health information may be used as necessary for your research-related treatment or to collect payment for your researchrelated treatment (when applicable). It may also be used to run the business operations of the institution. This information may be redisclosed or used for other purposes if a recipient described in this form is not required by law to protect the privacy

Tracking \#: H-21411

Approved On: 01/02/2009

Valid Through: 01/01/2010

Last Amended: 1/27/2009
Page 2 of 4

$\overline{\text { Initials }} \overline{\text { Date }}$ 
of the information.

You have a right to refuse to sign this authorization. Your health care outside the study, the payment for your health care, and your health care benefits will NOT be affected if you do not sign this form. However you will NOT be able to take part in the research study described in this authorization if you do not sign this form.

If you sign this authorization, you will have the right to cancel it at any time, except to the extent that UHA or UHA Affiliated, WVU, WVU Hospitals has already taken action based upon your authorization or needs the information to complete analysis and reports of data for this research study. This authorization will never expire until and unless you cancel it. To cancel this authorization, please write to the Principal Investigator, G.Greg ory Haff, Ph.D.,C.S.C.S.*D, FNSCA, at: Mailbox \#:P.O. Box 9227 You will be allowed to see or copy the information described on this form as long as the research is in progress, but you have a right to see and copy the information upon completion of the research in accordance with hospital policies.

The members and staff of any Institutional Review Board (IRB) that oversees this research study.

The Principal Investigator: G.Gregory Haff, Ph.D.,C.S.C.S.*D, FNSCA Members of UHA or UHA Affiliated, WVU, WVU Hospitals, administrative staff responsible for administering clinical trials and other research activities, including the Clinical Trials, Office/Office of Research and Sponsored Programs.

You have a right to receive a copy of this form after you have signed it.

Expiration Date: None

THE SUBJECT OR HIS/HER AUTHORIZED REPRESENTATIVE MUST BE PROVIDED WITH A COPY OF THIS FORM AFTER IT HAS BEEN SIGNED.

\section{SIGNATURE}

I have read this form and all of my questions about this form have been answered. By signing below, I acknowledge that I have read and accept all of the above.

Tracking \#: H-21411

Approved On: 01/02/2009

Valid Through: 01/01/2010

Last Amended: 1/27/2009
Page 3 of 4

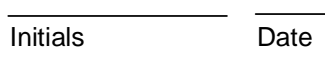


Signature of Subject or Authorized Representative Date

Print Name of Subject or Authorized Representative

Relationship of the person signing as Subject or Authorized Representative above to the Subject

Print Name of Individual Explaining this Research Authorization Form

\section{CONTACT INFORMATION}

The contact information of the subject or authorized representative who signed this form should be filled in below.

Address:

Telephone:

(daytime)

(evening)

E-mail Address (optional):

Tracking \#: H-21411

Approved On: 01/02/2009

Valid Through: 01/01/2010

Last Amended: 1/27/2009
Page 4 of 4

Initials

Date 
From: <wvuecomp@wvu.edu> [mailto:wvuecomp@wvu.edu]

Sent: 2009-01-02 11:52

To: Haff, G. Gregory; MCCRORY, JEAN; mmolina1@mix.wvu.edu; rruben@mix.wvu.edu

Subject: BRAAN2: New IRB Protocol Approved

The following new IRB Protocol has been approved.

Tracking \#: H-21411

PI: Haff, Gregory

Title: Validation of the use of accelerometers to determine vertical jump power outputs and displacements

Approval Date: 01/02/2009

Expiration Date: 01/01/2010

The BRAAN2 website can be accessed by clicking the following link: BRAAN2 Login 
From: <wvuecomp@wvu.edu> [mailto:wvuecomp@wvu.edu]

Sent: 2009-01-27 15:08

To: Haff, G. Gregory; MCCRORY, JEAN; kfowler1@mix.wvu.edu; mmolina1@mix.wvu.edu;

rruben@mix.wvu.edu; sburges1@mix.wvu.edu

Subject: BRAAN2: Amendment Approved

The following Amendment has been approved.

Tracking \#: AMEND-1429 (H-21411)

PI: Haff, Gregory

Title: Validation of the use of accelerometers to determine vertical jump power outputs and displacements

The BRAAN2 website can be accessed by clicking the following link: BRAAN2 Login 


\section{Medical/Health Questionnaire}

\section{Personal Information}

Today's Date Please print your name

How old are you? years

Sex: Male; Female

Please circle the highest grade in school you have completed:

$\begin{array}{lllllllll}\text { Elementary School } & 1 & 2 & 3 & 4 & 5 & 6 & 7 & 8 \\ \text { High School } & 9 & 10 & 11 & 12 & & & & \end{array}$

$\begin{array}{lllllllll}\text { College/Postgrad } & 13 & 14 & 15 & 16 & 17 & 18 & 19 & 20+\end{array}$

What is your marital status? Single; Married; Widowed; Divorced/Separated

Race or ethnic background:

White, not of Hispanic origin

American Indian/Alaskan native

Asian

Black, not of Hispanic origin

Pacific Islander

Hispanic

What is your job or occupation? Check the one that applies to the greatest percent of your time.

$\begin{array}{llc}\text { Health professional } & \text { Disabled, unable to work } & \text { Service } \\ \text { Manager, educator, professional } & \text { Operator, fabricator, laborer } & \text { Unemployed } \\ \text { Skilled crafts } & \text { Homemaker } & \text { Student } \\ \text { Technical, sales, support } & \text { Retired } & \text { Other }\end{array}$

\section{Symptoms or Signs Suggestive of Disease}

Place a check in the box if your answer is "yes."

1. Have you experienced unusual pain or discomfort in your chest, neck, jaw, arms, or other areas that may be due to heart problems?

2. Have you experienced unusual fatigue and/or shortness of breath at rest, during usual activities, or during mild-to-moderate exercise (e.g., climbing stairs, carrying groceries, brisk walking, cycling, etc.)?

3. Have you had any problems with dizziness or fainting?

4. When you stand up, or sometimes during the night while you are sleeping, do you have difficulty breathing?

5. Do you suffer from swelling of the ankles (ankle edema)?

6. Have you experienced an unusual and rapid throbbing or fluttering of the heart? 
7. Have you experienced severe pain in your leg muscles during walking?

8. Has a doctor told you that you have a heart murmur?

\section{Chronic Disease Risk Factors}

Place a check in the box if your answer is "yes."

9. Are you a male over age 45 years, or a female over age 55 years, or a female who has experienced premature menopause and is not on estrogen replacement therapy?

10. Has your father or brother had a heart attack or died suddenly of heart disease before age 55 years; has your mother or sister experienced these heart problems before age 65 years?

11. Has anyone in your family died suddenly before the age of 40 , excluding accidental death

12. Are you a current cigarette smoker?

13. Has a doctor told you that you have high blood pressure (more than $130 / 80 \mathrm{~mm} \mathrm{Hg}$ ), or are you on medication to control your blood pressure?

14. Is your total serum cholesterol greater than $200 \mathrm{mg} / \mathrm{dl}$, or has a doctor told you that your cholesterol is at a high risk level?

15. Do you have diabetes mellitus?

16. Are you physically inactive and sedentary (little physical activity on the job or during leisure time)?

17. During the past year, would you say that you experienced enough stress, strain, and pressure to have a significant effect on your health?

18. Do you eat foods nearly every day that are high in fat and cholesterol such as fatty meats, cheese, fried foods, butter, whole milk, or eggs?

19. Do you tend to avoid foods that are high in fiber such as whole grain breads and cereals, fresh fruits or vegetables?

20. Do you weigh 30 or more pounds than you should?

21. Do you average more than two alcoholic drinks each day? 


\section{Medical History}

22. Please check which of the following conditions you have had or now have. Also check medical conditions in your family (father, mother, brother(s), or sister(s)). Check as many as apply.

\section{Personal Family Medical Condition}

Coronary heart disease, heart attack, coronary artery surgery

Angina

High blood pressure

Peripheral vascular disease

Phlebitis or emboli

Other heart problems (specify:

Lung cancer

Breast cancer

Prostate cancer

Colorectal cancer (bowel cancer)

Skin cancer

Other cancer (specify:

Stroke

Chronic obstructive pulmonary disease (emphysema)

Pneumonia

Asthma

Bronchitis

Diabetes mellitus

Thyroid problems

Kidney disease

Liver disease (cirrhosis of the liver)

Hepatitis (A, B, C, D, or E)

Gallstones/gallbladder disease

Osteoporosis

Arthritis

Gout

Anemia (low iron)

Bone fracture

Major injury to foot, leg, knee, hip, or shoulder

Major injury to back or neck 
Stomach/duodenal ulcer

Rectal growth or bleeding

Cataracts

Glaucoma

Hearing loss

Depression

High anxiety, phobias

Substance abuse problems (alcohol, drugs, etc.)

Eating disorders (anorexia, bulimia)

Problems with menstruation

Hysterectomy

Sleeping problems

Allergies

HIV/AIDS

Any other health problems (please specify, and include information on any recent illnesses, hospitalizations, or surgical procedures):

23. Please check any of the following medications you currently take regularly. Also give the name of the medication.

\section{Medication}

Name of Medication

Heart medicine

Blood pressure medicine

Blood cholesterol medicine

Hormones

Birth control pills

Medicine for breathing/lungs

Insulin

Other medicine for diabetes

Arthritis medicine

Medicine for depression

Medicine for anxiety

Thyroid medicine

Medicine for ulcers

Pain killer medicine

Allergy medicine

HIV/AIDS medicine

Hepatitis medicine

Other (please specify) 


\section{Physical Fitness, Physical Activity/Exercise}

24. In general, compared to other persons your age, rate how physically fit you are:

Not at all

physically fit

4

some
Somewhat

physically fit
10

Extremely

physically fit

25. Outside of your normal work or daily responsibilities, how often do you engage in exercise that at least moderately increases your breathing and heart rate, and makes you sweat, for at least 20 minutes (such as brisk walking, cycling, swimming, jogging, aerobic dance, stair climbing, rowing, basketball, racquetball, vigorous yard work, etc.).

5 or more times per week $\quad 3$ to 4 times per week 1 to 2 times per week Less than 1 time per week Seldom or never

26. How much hard physical work is required on your job?
A great deal
A moderate amount
A little
None

27. How long have you exercised or played sports regularly?

I do not exercise regularly

$2-5$ years less than 1 year

$5-10$ years
1-2 years

more than 10 years

\section{Diet}

28. On average, how many servings of fruit do you eat per day? (One serving $=1$ medium apple, banana, orange, etc., $1 / 2$ cup of chopped, cooked, or canned fruit, $3 / 4$ cup of fruit juice).
none
1
2
3
4 or more

29. On average, how many servings of vegetables do you eat per day? (One serving $=1 / 2$ cup cooked or chopped raw, 1 cup raw leafy, $3 / 4$ cup of vegetable juice).
none
1-2
3
4
5 or more 
30. On average, how many servings of bread, cereal, rice, or pasta do you eat per day? (One serving = 1 slice of bread, 1 ounce of ready-to-eat cereal, $1 / 2$ cup of cooked cereal, rice, or pasta).
none
$1-3$
4-6
7-9 10 or more

31. When you use grain and cereal products, do you emphasize:

whole grain, high fiber mixture of whole grain and refined

refined, low fiber

32. On average, how many servings of red meat (not lean) do you eat per day? (One serving $=2-3$ ounces of steak, roast beef, lamb, pork chops, ham, burgers, etc.).
none
1
2
3
4 or more

33. On average, how many servings of fish, poultry, lean meat, cooked dry beans, peanut butter, or nuts do you eat per day? (One serving $=2-3$ ounces of meat, $1 / 2$ cup of cooked dry beans, two tablespoons of peanut butter, or 1/3 cup of nuts).

$\begin{array}{lllll}\text { none } & 1 & 2 & 3 & 4 \text { or more }\end{array}$

34. On average, how many servings of dairy products to you eat per day? (One serving = 1 cup of milk or yogurt, 1.5 ounces of natural cheese, 2 ounces of processed cheese).
none
1
2
3
4 or more

35. When you use dairy products, do you emphasize:

regular low-fat non-fat

36. How would you characterize your intake of fats and oils (e.g., regular salad dressings, butter or margarine, mayonnaise, vegetable oils).

High Moderate Low

\section{Body Weight}


37. How tall are you (without shoes)?

feet inches

38. How much do you weigh (minimal clothing and without shoes)? pounds

39. What is the most you have ever weighed? pounds

40. Are you NOW trying to:

Lose weight Gain weight Stay about the same

Not trying to do anything

\section{Psychological Health}

41. How have you been feeling in general during the past month?
In excellent spirits
In very good spirits
In good spirits mostly
I've been up and down in spirits a lot
In low spirits mostly
In very low spirits

42. During the past month, would you say that you experienced stress?

a lot of moderate relatively little almost none

43. In the past year, how much effect has stress had on your health:

a lot some hardly any or none

44. On average, how many hours of sleep do you get in a 24-hour period?

Less than $5 \quad 5$ to $6.9 \quad 7$ to $9 \quad$ More than 9

\section{Substance Use}

45. Have you smoked at least 100 cigarettes in your entire life?

Yes No

46. How would you describe your cigarette smoking habits? 
Never smoked

Used to smoke

How many years has it been since you smoked?

years

Still smoke

How many cigarettes a day do you smoke on average?

cigarettes/day

47. How many alcoholic drinks do you consume? (A "drink" is a glass of wine, a wine cooler, a bottle/can of beer, a shot glass of liquor, or a mixed drink).

Never use alcohol

1 per day
Less than 1 per week

2 to 3 per day
1 to 6 per week

More than 3 per day

\section{REFERENCES USED IN FORMULATING QUESTIONNAIRE}

1. National Center for Health Statistics, Stephens T and Schoenborn C. 1988. Adult health practices in the United States and Canada. Vital and Health Statistics. Series 5, No. 3. DHHS Public Health Service. Washington: U.S. Government Print Office.

2. Benson V and Marano MA. Current estimates from the National Health Interview Survey, 1993. National Center for Health Statistics. Vital Health Stat 10(190), 1994.

3. American School Health Association, Association for the Advancement of Health Education, Society for Public Health Education, Inc. The National Adolescent Student Health Survey: A Report on the Health of America's Youth. Oakland, CA: Third Party Publishing Company, 1989.

4. Nieman DC. Fitness and Sports Medicine: A Health-Related Approach. Palo Alto: Bull Publishing, 1995.

5. National Cancer Institute. DIETSYS Version 3.0, User's Guide: Health Habits and History Questionnaire. Bethesda, MD: National Cancer, Institute, 1993.

6. McDowell I and Newell C. Measuring Health: A Guide to Rating Scales and Questionnaires. New York: Oxford University Press, 1996.

7. American College of Sports Medicine. Guidelines for Graded Exercise Testing and Prescription. (5th edition). Philadelphia: Lea \& Febiger, 1995.

8. American College of Sports Medicine. Sol N and Foster C (eds). Health/Fitness Facility Standards and Guidelines. Champaign, IL: Human Kinetics Books, 1992.

9. U.S. Preventive Services Task Force. Guide to Clinical Preventive Services. (2nd edition). Alexandria, VA: International Medical Publishing, 1996. 


\section{Exercise History Questionnaire}

1. Are you an athlete?

a. If yes:

i. What sport do you participate in?

ii. How long have you been participating in this sport?

iii. How many times per week to you participate in this sport?

b. If no:

i. Do you exercise regularly?

1. If yes:

a. What types of exercise do you perform?

b. How often do you exercise?

c. How long have you been doing this?

2. If no:

a. When was the last time you participated in an exercise program?

2. Are you currently participating in a resistance training program?

a. If yes:

i. How long have you been resistance training?

ii. Is the back squat a part of your regular training program?

1. If yes:

a. How many times per week do you perform the back squat?

b. How long have you been performing the back squat as part of your resistance training program?

c. Currently what is the heaviest weight you have back squatted?

2. If no:

a. When is the last time you performed the back squat?

b. Do you have any injuries that preclude you from performing the back squat? 
b. If no:

i. Have you ever participated in a resistance training program?

1. If yes,

a. How long has it been since you participated in a resistance training program?

b. Was the back squat included in your resistance training regime?

3. Are you currently participating in a plyometric or jump training program?

a. If yes:

i. How long have you been performing jump/plyometric training?

ii. How many days per week do you perform jump/plyometric training?

b. If no:

i. Have you ever participated in a jump/plyometric training program?

\section{If Yes?}

a. How long has it been since you participated in a jump/plyometric training program?

4. Are you currently participating in a sport which requires a lot of jumping, such as volley ball, basket ball, hand ball etc.?

a. If yes:

a. What sport is it that you are participating in?

b. How often do you participate in this sport? 
VITA

RYAN M. RUBEN

\section{PERSONAL}

Birth Date: June $11^{\text {th }}, 1986$

Place of Birth: Marietta, $\mathrm{OH}$

Height: $1.78 \mathrm{~m}$

Weight: $75 \mathrm{~kg}$

\section{EDUCATION}

\begin{tabular}{llll}
\hline University & Emphasis & Degree & Date Received \\
\hline West Virginia University & Exercise Physiology & B.S. & 2008 \\
West Virginia University & Exercise Physiology & M.S. & In progress \\
\hline
\end{tabular}

Master's Thesis: Validity and Reliability of accelerometers for examining jump performance

TEACHING/RESEARCH/RELATED EXPERIENCE

2008-10 Graduate Assistant, West Virginia University School of Medicine, Morgantown, WV under the mentorship of G. Gregory Haff, Ph.D. Research included accelerometers and potentiation.

2008-10 Internship, West Virginia University Strength \& Conditioning Staff, Morgantown, WV.

\section{PUBLICATIONS}

Ruben RM, Bibbee CA, Childress MA, Harman MS, Reed KP, Haff GG. The acute effects of heavy-load squats on performance during plyometric jumps. J Strength Cond Res.

2010;24(2):358-69.

\section{MANUSCRIPTS IN PREPARATION OR SUBMITTED}

Ruben RM, Cormie P, Saffel H, McCrory JL, Bryner RW, Haff GG. Validity and reliability of accelerometers for measuring jump performance. 


\section{PROFESSIONAL ASSOCIATED MEMBERSHIPS}

2008-10 American Council of Exercise

2008-Present National Strength \& Conditioning Association

2009-Present American College of Sports Medicine

\section{CONFERENCE AWARDS}

$1^{\text {st }}$ Place Sport Science Poster. Third Annual Coaches \& Sport Science College, Johnson City TN, December 12-13, 2008.

\section{CONFERENCE PRESENTATIONS}

M. Molinari, K. Painter, R. Ruben, M.W. Ramsey, M.E. Stone, G.G. Haff, and M.H. Stone. A comparison of daily undulating with traditional periodization in collegiate track and field athletes. Third Annual Coaches \& Sport Science College, Johnson City TN, December 12-13, 2008 .

G.G. Haff, M. Molinari, R. Ruben, M.W. Ramsey, K. Painter, M.E. Stone, and M.H. Stone. A pilot study of the reliability and validity of accelerometer based vertical jump assessments. Third Annual Coaches \& Sport Science College, Johnson City TN, December 12-13, 2008.

R. Ruben, M. Molinari, C. Bibbee, M. Childress, M. Harman, K. Reed, J. Thomas, and G. G. Haff. A pilot study on the effects of heavy-loaded squats on performance during plyometric jumps. Third Annual Coaches \& Sport Science College, Johnson City TN, December 12-13, 2008.

R. Ruben, M. Molinari, C. Bibbee, M. Childress, M. Harman, K. Reed, J. Thomas, and G. G. Haff. The effects of heavy-loaded squats on performance during plyometric jumps. NSCA National Conference, Las Vegas NV, July 8-11, 2009. 